\title{
2 Wohnungsbewertungsmodell ohne Einkommensteuern
}

\subsection{Bewertung zu Entscheidungszwecken}

\subsubsection{Grundstück der funktionalen Bewertungstheorie}

Die funktionale Bewertungstheorie folgt dem Gedanken, daß es keinen Wert an sich gibt, der einer Sache wie eine Eigenschaft anhaftet und für jedermann gültig ist. ${ }^{64}$ Vielmehr unterscheidet sie je nach Aufgabenstellung und verfolgtem Zweck verschiedene Wertarten. ${ }^{65}$ Die Aufgabe im Rahmen der Entscheidungsfunktion lautet, die Grenze der Konzessionsbereitschaft bezüglich der Konditionen des Eigentumsübergangs aus Sicht des Bewertungssubjekts zu ermitteln. ${ }^{66}$ Der ermittelte Entscheidungswert stellt aus Sicht des präsumtiven Käufers die Obergrenze für den Kaufpreis dar, bis zu dem ein Kauf gerade noch nicht nachteilig ist. Aufgrund der Subjektivität jedes Wertes unterscheidet sich der Grenzpreis von Person zu Person. Anstatt einen bestimmten Rechenweg vorzuschreiben, ${ }^{67}$ folgt die funktionale Bewertungstheorie vier lebensnahen Prinzipien, die sich am Bewertungsobjekt Eigentumswohnung besonders gut erklären lassen:

${ }^{64}$ Kritisch zu vermeintlich objektiven Wertvorstellungen äußert sich SIEBEN: „Was sollten die [...] beteiligten Parteien mit einer Wertgröße anfangen, die von allen individuellen Gegebenheiten abstrahiert?“, SIEBEN (1963), S. 80. „Der Informationsgehalt einer zweckspezifischen Größe (Wert) ist höher als einer Größe, die Allgemeingültigkeit beansprucht (fairer Wert)“", ergänzt MATSCHKE (1976), S. 517, während ein objektivierter Wert überhaupt keiner Aufgabe gerecht wird, vgl. MOXTER (1977), S. 253. MATSCHKE konstatiert: „Interessiert sich doch niemand dafür, was jedermann für die Unternehmung zahlen bzw. verlangen könnte, sondern die Person [...], die die Unternehmung bewerten lassen will, möchte wissen, was sie höchstens zahlen darf.“ MATSCHKE (1969), S. 58. „Vor allem ist der Sinn einer Wertgröße fraglich, die für jedermann gleichermaßen gültig sein soll, in Wirklichkeit aber wegen ihrer Schablonisierung für keine der an der Bewertung interessierten Parteien [...] Gültigkeit besitzen kann."MÜNSTERMANN (1970), S. 24.

65 Wenn der Bewertungsanlaß eine Eigentumsänderung ist, befindet man sich im Bereich der Hauptfunktionen Entscheidung, Vermittlung und Argumentation, vgl. MATSCHKE/BRÖSEL (2013), S. 52 ff., HERING/TOLL (2017a), S. 184. Da alle anderen Werte von ihm abhängen, ist der Entscheidungswert die „zentrale Größe der Unternehmensbewertung“, so TOLL (2011), S. 1, ähnlich BRÖSEL/ HAUTTMANN (2007), S. 233, ZWIRNER/PETERSEN (2017a), S. 181. Zum Argumentationswert vgl. MATSCHKE (1976), MATSCHKE/MUCHEYER (1977), GORNY (2002), BRÖSEL (2004), HERING/BRÖSEL (2004), zum Vermittlungswert vgl. KÖNIG (1977), MATSCHKE (1979), ZWIRNER/PETERSEN (2017b).

${ }^{66}$ Auch zum Folgenden vgl. HERING/OLBRICH (2002), S. 148, BRÖSEL/TOLL (2011), S. 260.

67 Vgl. SIEBEN/DÖRNER/KÖNIG/MATSCHKE/MOXTER/SIELAFF/ZAPF (1977), S. 277, MOXTER (1992), S. 54.

(C) Der/die Autor(en) 2021

S. Walochnik, Bewertung von Eigentumswohnungen,

Finanzwirtschaft, Unternehmensbewertung \& Revisionswesen,

https://doi.org/10.1007/978-3-658-30840-7_2 
- Zukunftsbezug: ${ }^{68}$ Der Wert ist ein subjektives Urteil über die zukünftigen, von der Eigentumswohnung hervorgerufenen Mietüberschüsse: ${ }^{69}$ Sie ist Mittel zum Zweck, um zukünftige Zahlungsströme zu generieren. ROTTKE und KRAUTZ sprechen zutreffend davon, daß man nicht in Immobilien, sondern in Mietverträge investiert. ${ }^{70}$ Selbst bei geplanter Eigennutzung der Wohnung sind zukünftige Mietüberschüsse der anzulegende Maßstab, weil man die ersparte Opportunitätsmiete als Vergleichsmaßstab anlegen muß. ${ }^{71}$ Die Zukunft ist unbekannt und so werden vergangene Erfolge oft als Indikator für das verstanden, was man in Zukunft aus dem Bewertungsobjekt erwarten kann. Vergangenheitsdaten können Anhaltspunkte für zukünftige Entwicklungen liefern. ${ }^{72}$ Sie sollten aber nicht undifferenziert in die Zukunft hochgerechnet werden. Man muß die Verhältnisse der Vergangenheit reflektieren und kritische Überlegungen hinsichtlich ihres Fortbestands anstellen. ${ }^{73}$ Gab es z.B. Ausfälle aufgrund eines zahlungsunwilligen Mieters, der inzwischen aus der Wohnung entfernt werden konnte, oder gab es einmalige, kostenintensive Sanierungsarbeiten, sollte man seine Prognose um derartige Sonderfaktoren bereinigen.

- Gesamtbewertung: ${ }^{74}$ Der von der Wohnung ausgehende Nutzen entspringt ihrer wirtschaftlichen Einheit und nicht der Summe ihrer Einzelteile, schließlich sind

68 Zum Zukunftserfolgswert vgl. BUSSE VON COLBE (1957). Zum Prinzip des Zukunftsbezugs vgl. LEUTHIER (1988), S. 30 f., MEYERING (2007), S. 97, MATSCHKE/BRÖSEL (2013), S. 172, GLEIßNER (2017), S. 928, in Bezug auf Immobilien HARES (2011), S. 97. „Für das Gewesene gibt der Kaufmann nichts", sagt ein geläufiges Sprichwort, zitiert nach HERING (2014), S. 20.

69 Vgl. ROPETER (1998), S. 69, BONE-WINKEL/FOCKE/SCHULTE (2016), S. 9, VON BRESSENSDORF (2016), S. 246. In Bezug auf Grundstücke vgl. bereits SCHMALENBACH (1947), S. 89. Dieses Verständnis des Wertbegriffs ist vor allem in der Unternehmensbewertungstheorie allgemein bekannt, vgl. BERLINER (1913), S. 25, MELLEROWICZ (1952), S. 19 ff., SIEBEN/ZAPF (1981), S. 19, TSCHÖPEL (2004), S. 97, MATSCHKE (2017a), S. 15.

70 Vgl. ROTTKE/KRAUTZ (2017), S. 777 f.

71 Vgl. LEIFELS/RAFFELHÜSCHEN (2014), S. 135, TOLL/WALOCHNIK (2013), S. 21, HERTWECK (2017), S. 246 f. Daß die Vorteilhaftigkeit auch bei Selbstnutzung durch zukünftige Zahlungsströme determiniert wird, zeigt sich spätestens bei späterem Verkauf der Wohnung, ,denn bei dem neuen Käufer wird er voraussichtlich dem gleichen Interesse begegnen." SCHMALENBACH (1917), S. 1, dort in Bezug auf eine „Wirtschaftsanlage“. Bereits MOXTER (1983), S. 79 nennt die Entnahme von Brennholz durch einen Forstbesitzer als Beispiel zur Äquivalenz zwischen Einzahlung und (Konsum-) Auszahlungsersparnis. Zum Substanzwert als vorgeleistete Ausgaben vgl. SIEBEN (1992), S. 69 f.

72 Vgl. SERFLING/PAPE (1995), S. 814 f., HENSELMANN (1999), S. 112, MEYERING (2007), S. 107 f. Die „Zukunft ist stets das Kind der Gegenwart und das Kindeskind der Vergangenheit“, sagte MÜNSTERMANN (1970), S. 49. Analyseschritte zur Prognose von Eingangsdaten und Schätzung des Zukunftserfolgs zeigen BRÖSEL/DECHANT (2003), S. 146 ff., VON AHSEN/DE WITT (2009), S. 138 ff.

73 Warnend vor der unreflektierten Hochrechnung von Vergangenheitsdaten äußern sich RICHTER (1942), S. 107, BUSSE VON COLBE (1957), S. 179, MATSCHKE (1979), S. 121.

74 Zum Prinzip der Gesamtbewertung vgl. MELLEROWICZ (1952), S. 18 f., MATSCHKE (1972), BUSSE VON COLBE (1992). 
diese in der Immobilie gebunden: ${ }^{75}$ Es werden mit dem Mieter aber keineswegs einzelne Mietverträge für Fenster, Wohnungstüren, Deckenlampen oder für die einzelnen Zimmer abgeschlossen, sondern ein Mietvertrag für sein gesamtes Zuhause. Nur als Ganzes entfaltet das Bewertungsobjekt seinen vollen Nutzen einschließlich Synergien, erst „,durch das Zusammenwirken der Teile ergeben sich dann wertsteigernde Effekte" ${ }^{676}$.

- Subjektivität: ${ }^{77}$ Die funktionale Bewertungstheorie trägt dem Gedanken Rechnung, daß verfolgte Ziele (das Zielsystem) von Person zu Person ebenso unterschiedlich sind wie alternative Handlungsmöglichkeiten, Restriktionen und Wechselwirkungen (das Entscheidungsfeld). ${ }^{78}$ Abhängig von diesem besitzt jeder Wert nur in einer ganz bestimmten Subjekt-Objekt-Objekt-Relation Gültigkeit. ${ }^{79}$ Möglichkeiten und Konditionen von Kreditaufnahme und Geldanlage unterscheiden sich von Person zu Person, man denke nur an den Immobilienkredit. ${ }^{80}$ Die erzielbaren Mietüberschüsse sind stets das Ergebnis individueller Prognose, selbst wenn man mit einwertigen Daten rechnet. ${ }^{81}$ Überdies hängen Erfolg und Konditionen einer Neuvermietung vom Verhandlungsgeschick beim Abschluß des Mietvertrags und damit von Erfahrung und Menschenkenntnis des Vermieters ab. Möglicherweise sind seine Ausstrahlung und Vorgehensweise auch entscheidend dafür, ob eine strittige Forderung vom Mieter bezahlt wird oder nicht. Neben personenbezogenen Faktoren ist auch jede Wohnung ein Unikat, deren Nutzen und Vermietbarkeit individuell durch Objekt- und Standortfaktoren beeinflußt werden. ${ }^{82}$

75 Vgl. MORAL (1919), S. 10, ALBACH (1962), S. 68, SCHMALENBACH (1966), S. 60.

76 MATSCHKE/BRÖSEL (2013), S. 4. Vgl. dazu auch ENGELEITER (1970), S. 15, SIEBEN (1977), S. 66, WAMELING (2004), S. 47 ff., MEYERING (2007), S. 100. „Wenn das gesamte Unternehmen nur so viel wert ist wie die Summe der Einzelteile, dann ist der wirtschaftliche Zweck verfehlt", sagte SCHMALENBACH (1917), S. 10, ähnlich BANKMANN (1960), S. 15. Synergien lassen ,sich plastisch als $2+2$ = 5-Effekt bezeichnen“, verdeutlichen BALLWIESER/HACHMEISTER (2016), S. 14. Beispiele für Synergien im Rahmen der Unternehmensakquisition gibt OLBRICH (1999), S. 41 ff.

77 Zum Prinzip der Subjektivität vgl. bereits GOSSEN (1854), S. 24, MENGER (1871), S. 106. Insbesondere zur Subjektivität bei der Bewertung vgl. LEUTHIER (1988), S. 38 ff., $\operatorname{HENSELMANN}(1999)$, S. 20.

78 SIEBEN definiert das Zielsystem als „Absichten (das Wollen)“ und das Entscheidungsfeld als „Möglichkeiten (das Können) des [...] Bewertungsinteressenten.“ SIEBEN (1976), S. 497, ähnlich ADAM (2000), S. 37, TOLL (2011), S. 33, JAPES (2011), S. 9 f., HERING (2014), S. 27, BAMBERG/COENEN$B E R G / K R A P P$ (2019), S. 15.

79 Vgl. BALLWIESER (2002), S. 71, HERING/OLBRICH (2003), S. 1579, MATSCHKE/BRÖSEL (2013), S. 166.

${ }^{80}$ Selbst das vielversprechendste Bewertungsobjekt muß einen Wert von null haben, wenn der Käufer über keine eigenen Mittel verfügt und niemand ihm Kredit gibt, sagte MATSCHKE (1975), S. 276.

81 Vgl. ENGELEITER (1970), S. 81, KLINGELHÖFER (2006), S. 71, HERING (2017), S. 139 f.

82 Vgl. TOLL/WALOCHNIK (2013), S. 34, BONE-WINKEL/FOCKE/SCHULTE (2016), S. 17 f. 
- Zweckbezug: ${ }^{83}$ Bereits SCHMALENBACH sagte, daß jede Rechnung ihrem Zweck entsprechend gestaltet sein muß ${ }^{84}$ Während die drei vorgenannten Prinzipien der älteren, subjektiven Wertlehre entstammen, ergänzt die funktionale Bewertungstheorie diese um den fehlenden vierten Baustein. ${ }^{85}$ Der Wert ist nicht bloß in einer ganz bestimmten Subjekt-Objekt-Objekt-Relation gültig; er ist darüber hinaus sogar bei ein und demselben Bewertungssubjekt unterschiedlich, je nachdem, welchem Zweck eine Bewertung dient. ${ }^{86}$ Es leuchtet unmittelbar ein, daß für unterschiedliche Zwecke unterschiedliche Rechenverfahren benutzt werden, die wiederum zu unterschiedlichen Ergebnissen führen. ${ }^{87}$

Abbildung 1 faßt das Gesagte zusammen.

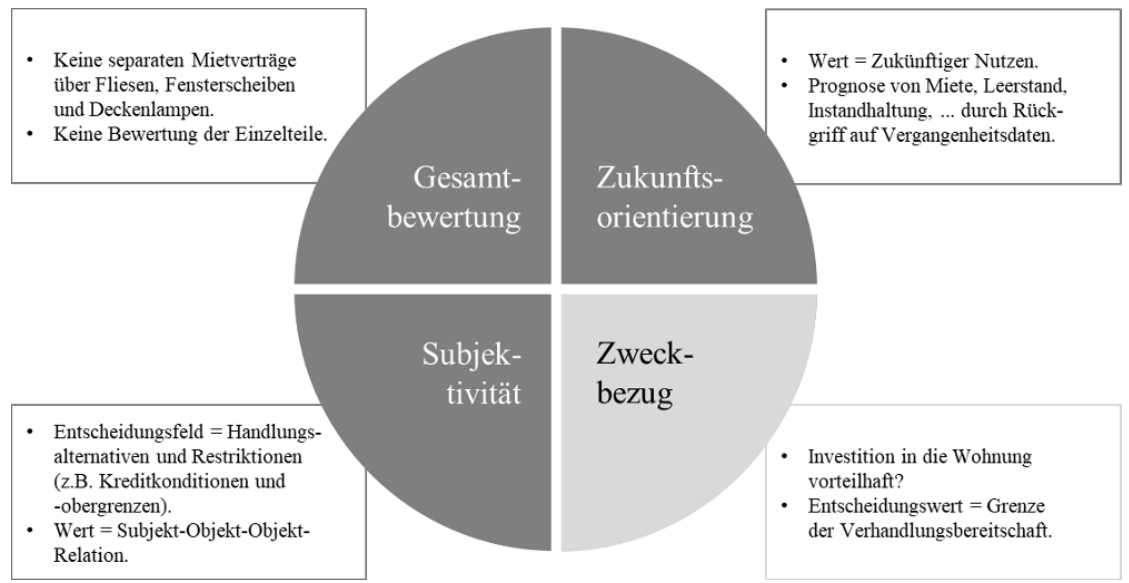

Abbildung 1: Prinzipien der funktionalen Bewertungstheorie

83 Zum Prinzip des Zweckbezugs vgl. bereits WOLF (1886), S. 431, vgl. auch SCHMALENBACH (1962), S. 90, MATSCHKE (1976), S. 517, SIEBEN/DÖRNER/KÖNIG/MATSCHKE/MOXTER/SIELAFF/ZAPF (1977), S. 264 und S. 292, BRÖSEL/HAUTTMANN (2007), S. 227, BRÖSEL/TOLL (2016), S. 38.

${ }^{84}$ Vgl. SCHMALENBACH (1963), S. 141. Zuvor pointierte er treffend: „Daß man eine Länge nicht mit einem Gummiband messen kann, weiß Jedermann.“ SCHMALENBACH (1947), S. 24.

85 Vgl. HERING (2004), S. 108 f., MATSCHKE/BRÖSEL (2013), S. 23 f., MATSCHKE (2017b), S. 33 f.

86 Zur Zweckabhängigkeit führt SCHMALENBACH aus: „Wenn man im Gespräch die Frage aufwirft, was ein bestimmter Gegenstand wert sei, so kann der Grund dieser Frage verschieden sein. Der Eine will wissen, was er anlegen muß, um einen gleichen Gegenstand zu kaufen, der Andere möchte den Gegenstand verkaufen und einem Dritten geht es lediglich darum, einem ihm innewohnenden Schätzungsbedürfnis zu genügen." SCHMALENBACH (1947), S. 28.

87 Man denke an den Beleihungswert der Wohnung als Kreditsicherheit aus Sicht der Bank oder die behördlich-normierte Berechnung des Einheitswertes als Bemessungsgrundlage der Grundsteuer. 


\subsubsection{Fundament der investitionstheoretischen Unternehmensbewertung}

\subsubsection{Quantitative Interpretation der Eigentumswohnung als Zahlungsstrom}

Weil aus finanzwirtschaftlichem Blickwinkel jedes Bewertungsobjekt auf seinen mathematisch greifbaren Zahlungsstrom reduziert wird, sind Modelle der investitionstheoretischen Unternehmensbewertung ebensogut auf Immobilien, Eigentumswohnungen und sonstige Bewertungsobjekte anwendbar. ${ }^{88}$ Unternehmen sind häufig angeführte, idealtypische Prototypen von Bewertungsobjekten, denen zukünftige Erfolge zugerechnet werden können. ${ }^{89}$ Aus investitionstheoretischer Sicht wird jedes Bewertungsobjekt, sei es nun ein Unternehmen oder eine Eigentumswohnung, auf seine mathematisch greifbaren Bestandteile in Form von Geldbewegungen reduziert: ${ }^{90}$ Da man diese Zahlungsströme auf einem Kontoauszug - zumindest ex post - zweifelsfrei nachvollziehen kann, besteht bei dieser Rechengröße im Vergleich zu bilanziellen Größen kein Spielraum für Manipulationen. ${ }^{91}$

Damit sind Eigentumswohnungen, Unternehmen und alle weiteren Bewertungsobjekte aus investitionstheoretischer Sicht nur Surrogate für die aus ihnen entspringenden Überschüsse, die man bei Kauf des Investitionsobjekts im Vergleich zur Nichtdurchführung zusätzlich entnehmen und konsumieren kann. ${ }^{92}$ Als Mittel zum Zweck dienen sie dem

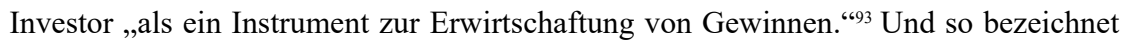
VON BRESSENSDORF den Mietvertrag plakativ als „Cashflow-Generator [, der] zwar nicht die Umwandlung von Blei in Gold, aber immerhin die Umwandlung von Stein, Stahl und Beton in klingende Münze bewerkstelligt. “994

${ }^{88}$ Forschungsbeiträge zur Immobilienbewertung lieferten u.a. KEUPER/PAPE (2008), PAPE (2009), HARES (2011), S. 102 ff., TOLL/WALOCHNIK (2013), dort wurden jedoch keine Eigentumswohnungen fokussiert, und es erfolgte auch keine Berücksichtigung personenbezogener Einkommensteuern. Beiträge zu aus anderen Bereichen stammenden Bewertungsobjekten findet man bspw. zu audiovisuellen Medienrechten, vgl. BRÖSEL (2002), Telekommunikationsunternehmungen, vgl. BRÖSEL/ DECHANT (2003), Umweltschutzinvestitionen, vgl. KLINGELHÖFER (2006), Mehrstimmrechtsaktien, vgl. HERING/OLBRICH (2003) oder Schäden durch fehlerhafte Entscheidungswertermittlung, vgl. WASMUTH (2018).

89 Vgl. SIEBEN (1967), S. 126 f., BYSIKIEWICZ (2008), S. 51, MATSCHKE/BRÖSEL (2013), S. 12.

90 Vgl. ALVANO (1988), S. 106, HERING (2014), S. 32, ähnlich bereits GUTENBERG (1987), S. 2. TOLL formuliert treffend: „Im Falle eines Unternehmensverkaufs [...] gibt der Verkäufer sein Eigentum an der Unternehmung zugunsten des Käufers auf und erhält im Gegenzug eine - in der Regel monetäre - Gegenleistung.“ TOLL (2011), S. 3. Zur Vereinfachung bezieht man Geldbewegungen saldiert auf das jeweilige Periodenende, vgl. HAX (1985), S. 11 f., MANDL/RABEL (1997), S. 79, TER HORST (2009), S. 38, BALLWIESER/HACHMEISTER (2016), S. 15.

91 Vgl. BRÖSEL (2002), S. 75, BRÖSEL/DECHANT (2003), S. 144, JAPES (2011), S. 18.

92 Vgl. AXER (1932), S. 5 f., MOXTER (1977), S. 254, LEUTHIER (1988), S. 25 f., SCHNEIDER (1992), S. 1, OBERMAIER (2004), S. 7 f., ROLLBERG (2008), S. 24.

93 SIEBEN (1977), S. 58.

94 VON BRESSENSDORF (2016), S. 246, ähnlich ROPETER (1998), S. 69, ROTTKE/KRAUTZ (2017), S. $777 \mathrm{f}$. 


\subsubsection{Bewertung als Vergleich mit Alternativgeschäften}

Weil die Geldbewegungen der Eigentumswohnung in unterschiedlicher Höhe zu unterschiedlichen Zeitpunkten anfallen, werden sie durch Auf- bzw. Abzinsung zu einem zeitlich aggregierten Gegenwartswert umgeformt. ${ }^{95}$ Dies geschieht durch gedankliche Gegengeschäfte in Form von Kreditaufnahmen oder Geldanlagen.

Die Funktionsweise sei an einem didaktisch stark vereinfachten Beispiel vorgeführt: ${ }^{96}$ Es sei angenommen, der Planungshorizont bestehe aus lediglich drei Perioden und es gebe nur einen Sollzinssatz $i_{S}$ für endfällige Kreditaufnahmen jeglicher Laufzeit. Auch sei davon ausgegangen, daß dem Investor im Zeitpunkt der Entscheidung $t=0$ keinerlei liquide Mittel zur Verfügung stehen, er jedoch aus dem Bewertungsobjekt mit Sicherheit einen Zahlungsstrom von $(0 ;+3.536,10 ;+3.536,10 ;+3.536,10)$ erwarten kann, sofern er es erwirbt. Durch Kreditaufnahme ist es ihm möglich, die Zahlungsüberschüsse aller drei Perioden durch Gegengeschäfte vollständig auszugleichen: Er kann in $t=0$ Kredite für verschiedene Laufzeiten in Höhe von jeweils $g_{t} \cdot\left(1+i_{S}\right)^{-t}$ aufnehmen, für die er neben der endfälligen Tilgung in den Zeitpunkten $t$ auch Schuldzinsen für t Jahre zahlen muß. Beispielsweise könnte er in $t=0$ einen Kredit von 3.432,10 EUR aufnehmen, um in $\mathrm{t}=3$ einen Betrag von 3.536,10 EUR zurückzuzahlen. Tabelle 1 zeigt vorzunehmende Gegengeschäfte für alle drei Zeitpunkte.

\begin{tabular}{|c|c|c|c|c|}
\hline Zins & $\mathbf{t}=\mathbf{0}$ & $t=1$ & $t=2$ & $t=3$ \\
\hline & $\mathbf{0 , 0 0}$ & $3.536,10$ & $3.536,10$ & $3.536,10$ \\
\hline $1 \%$ & $3.536,10 \cdot 1,01^{-3}=3.432,10$ & & & $-3.536,10$ \\
\hline $1 \%$ & $3.536,10 \cdot 1,01^{-2}=3.466,42$ & & $-3.536,10$ & $\mathbf{0 , 0 0}$ \\
\hline $1 \%$ & $3.536,10 \cdot 1,01^{-1}=3.501,09$ & $-3.536,10$ & $\mathbf{0 , 0 0}$ & \\
\hline $\mathbf{E}$ & $10.399,62$ & $\mathbf{0 , 0 0}$ & & \\
\hline
\end{tabular}

Tabelle 1: Umformung von Zahlungsströmen zum Ertragswert ${ }^{97}$

Die Rückzahlungen der Kredite addieren sich jeweils mit den Zahlungsüberschüssen des Bewertungsobjekts zu null, daher spricht HERING passenderweise von ihrem Eindampfen. ${ }^{98}$ Aufgrund der drei Kreditgeschäfte steht dem Investor in $\mathrm{t}=0$ ein Betrag von 10.399,62 EUR zur Verfügung. Würde er das vollständig kreditfinanzierte Bewertungsobjekt zu diesem Preis erwerben, trägt es sich gerade noch selbst. Dieser Ertragswert ist sein maximal zahlbarer Grenzpreis.

\footnotetext{
95 Zu Folgendem vgl. BUSSE VON COLBE (1957), S. 57, LAUX/FRANKE (1969), S. 206, MOXTER (1983), S. 123 ff., $\operatorname{HAX}$ (1985), S. 12, HERING (2017), S. 35 ff. „Die Diskontierung soll [...] also nur die störende Zeitkomponente ausschalten." ALBACH (1962), S. 56.

96 Folgendes Beispiel ist angelehnt an HERING (2017), S. 35 ff. und S. 66 ff.

97 Ähnlich HERING (2017), S. 41.

98 HERING (2017), S. 36.
} 


\subsubsection{Unvollkommener Kapitalmarkt als realitätsnahe Modellumgebung}

Der Maßstab, an dem sich das Bewertungsobjekt messen lassen muß, ist das günstigste Gegengeschäft einer jeweiligen Periode. ${ }^{99}$ Aus diesen sogenannten Grenzobjekten leiten sich die zur Abzinsung benötigten Kalkulationszinsfüße her, die man Grenzzinsen oder Lenkpreise nennt. ${ }^{100}$

Im vorgenannten Beispiel existierte ausschließlich der einprozentige Kreditzins. Da aber die reale Welt, in der sich der Kauf einer Eigentumswohnung abspielt, komplexer und unvollkommener als der lehrbuchartig vereinfachte Fall ist, existieren eine große Menge potentieller Gegengeschäfte - und zudem zahlreiche Interdependenzen: ${ }^{101}$ Erst aus der Gesamtbetrachtung des optimalen Investitions- und Finanzierungsprogramms ergeben sich die periodenspezifischen Grenzobjekte - und werden dann bereits nicht mehr zur Entscheidungsfindung benötigt. ${ }^{102}$ Dies ist das ,große Dilemma der Lenkpreistheo$r i e^{\text {“103 }}$. Weil bspw. die Finanzierung einer Eigentumswohnung meist durch Kreditaufnahme erfolgt, ${ }^{104}$ können sich die Grenzobjekte aufgrund des Kaufs ändern. ${ }^{105}$ Vielleicht ist es nun ratsam, eine Sondertilgung auf den teuren Kredit zu leisten, während die beste Alternative ohne Kauf der Wohnung nur eine zinslose Kassenhaltung gewesen wäre.

Obwohl jedes Modell dem Prinzip der isolierenden Abstraktion folgt, ist die Wahl einer realitätsnahen Modellumgebung obligatorisch, wenn man reale Entscheidungen durch mathematische Berechnungen unterstützen möchte. Es bietet sich der unvollkommene

99 Auch zum Folgenden vgl. ALBACH (1962), S. 81, JAENSCH (1966), S. 661, LAUX/FRANKE (1969), S. 218, MATSCHKE (1972), S. 152, MOXTER (1975), S. 143 ff., ADAM (2000), S. 294, HERING (2002), S. 18. „Der Zins auf dem unvollkommenen Kapitalmarkt drückt daher eine entgangene Gelegenheit aus.“ MATSCHKE/MATSCHKE (1993), S. 249.

${ }^{100} \mathrm{Zu}$ den erheblichen Auswirkungen des Kalkulationszinsfußes auf das Rechenergebnis vgl. SCHMALENBACH (1966), S. 50, ENGELEITER (1970), S. 36, MÜNSTERMANN (1970), S. 63.

101 Vgl. ROPETER (1998), S. 10, ROTTKE (2017b), S. 121, BRAUER (2019a), S. 10.

102 Vgl. LEUTHIER (1988), TOLL (2011), S. 37 f., HERING (2017), S. 142 ff., BRÖSEL (2017), S. 212. Ähnlich bereits MIRRE: „Es ist nötig, sämtliche Möglichkeiten, aus der Sache einen Nutzen zu ziehen, zu betrachten, aus ihnen die beste zu wählen [...]. Der höchste Geldbetrag ist dann der subjektive Wert.“MIRRE (1913), S. 158.

${ }^{103}$ MATSCHKE/HERING/KLINGELHÖFER (2002), S. 212.

${ }^{104}$ Vgl. JOKL (2009), S. 63 f., SCHÄFERS/HOLZMANN/SCHULTE/LANG/SCHOLZ (2016), S. 483 ff., MARKMANN/MARKMANN/ROTTKE (2017), S. 649.

${ }^{105}$ Der Zukauf des Bewertungsobjekts führt meist zur Umstrukturierung des Investitions- und Finanzierungsprogramms und damit zu einer Veränderung der Grenzobjekte. Der Grenzpreis umfaßt dann zusätzlich zum Ertragswert des Bewertungsobjekts auch den Mehrwert aus der Umstrukturierung, vgl. ROLLBERG (2001), S. 62, KLINGELHÖFER (2006), S. 88, HERING (2014), S. 201, MATSCHKE (2017c), S. 65, HERING/TOLL (2017b), S. 200 f. 
Kapitalmarkt an, dessen Annahmen für reale Entscheidungen brauchbar sind. ${ }^{106}$ Dessen Merkmale zeigt Tabelle 2. ${ }^{107}$

\begin{tabular}{|c|c|c|}
\hline $\begin{array}{l}\text { Beobachtung aus } \\
\text { der Realität }\end{array}$ & $\begin{array}{l}\text { Resultierende } \\
\text { Modellannahme }\end{array}$ & Auswirkung auf die Bewertungssituation \\
\hline $\begin{array}{l}\text { Existenz von Trans- } \\
\text { aktionskosten, Ban- } \\
\text { ken und subjektiven } \\
\text { Präferenzen }\end{array}$ & $\begin{array}{l}\text { Sollzins } \geq \text { Haben- } \\
\text { zins, Verschiedene } \\
\text { Konsumentnahme- } \\
\text { muster }\end{array}$ & $\begin{array}{l}\text { Wegfall der FISHER-Separation: Unterschied- } \\
\text { liche Konsumentnahmemuster können nicht } \\
\text { ohne weiteres durch Finanztransaktionen in- } \\
\text { einander überführt werden. Sie sind mit der } \\
\text { Wahl des optimalen Investitions- und Finan- } \\
\text { zierungsprogramms interdependent. }\end{array}$ \\
\hline $\begin{array}{l}\text { Zwischen Objekten } \\
\text { bestehende Syner- } \\
\text { gien, Abhängigkei- } \\
\text { ten und Ausschlüsse }\end{array}$ & Interdependenzen & $\begin{array}{l}\text { Dilemma der Lenkpreistheorie: Der perio- } \\
\text { denspezifische Steuerungszins ergibt sich } \\
\text { modellendogen aus den gerade noch teils rea- } \\
\text { lisierten Objekten des optimalen Programms. } \\
\text { Er fällt erst gleichzeitig mit der Lösung des } \\
\text { Totalmodells an, ist zur Lösung dann aber } \\
\text { überflüssig. }\end{array}$ \\
\hline $\begin{array}{l}\text { Unterschiede in Ver- } \\
\text { schuldungsgraden, } \\
\text { Bonität, Markt- } \\
\text { macht, Erwartungen } \\
\text { und Informations- } \\
\text { stand }\end{array}$ & $\begin{array}{l}\text { Risikozuschläge, } \\
\text { unterschiedliche } \\
\text { Zinskonditionen }\end{array}$ & $\begin{array}{l}\text { Subjektivität: Die Vorteilhaftigkeit einer In- } \\
\text { vestition ist aus subjektiver Sicht zu beurtei- } \\
\text { len. }\end{array}$ \\
\hline $\begin{array}{l}\text { Insolvenzrisiko, } \\
\text { knappe Kreditsicher- } \\
\text { heiten }\end{array}$ & Kreditobergrenzen & $\begin{array}{l}\text { Nicht jedes vorteilhafte Objekt kann voll } \\
\text { bzw. überhaupt realisiert werden. }\end{array}$ \\
\hline Unteilbare Objekte & Ganzzahligkeit & $\begin{array}{l}\text { Bei unteilbaren Grenzobjekten existieren } \\
\text { keine Steuerungszinsen. Die Lenkpreistheo- } \\
\text { rie versagt. }\end{array}$ \\
\hline Steuerpflicht & $\begin{array}{l}\text { Minderung poten- } \\
\text { tieller Entnahmen }\end{array}$ & Modifikation der Zahlungsströme. \\
\hline
\end{tabular}

Tabelle 2: Merkmale des unvollkommenen Kapitalmarkts

${ }^{106}$ Vgl. HAX (1964), S. 430, BALLWIESER (1990), S. 30, TOLL/HERING (2017), S. 461 f. Hingegen geht der vollkommene Kapitalmarkt von Prämissen aus, die in der Realität nicht zu finden sind, z.B. homogene Erwartungen und die Abwesenheit von Transaktionskosten und Steuern. Es handelt sich um eine idealisierte Modellumgebung für Erklärungszwecke, die für Entscheidungen hinsichtlich realer Problemstellungen unbrauchbar ist, vgl. ALTROGGE (1996), S. 38, HERING/OLBRICH/ROLLBERG (2010), S. 40, BRÖSEL/TOLL (2011), S. 262. Bereits SCHMALENBACH ging es ,nicht darum, den Preis zu erklären, sondern [...] vielmehr darum, die optimale Geltungszahl zu bestimmen." SCHMALENBACH (1947), S. 23.

${ }^{107} \mathrm{Zu}$ den Merkmalen des unvollkommenen Kapitalmarkts vgl. ALBACH (1962), S. 31 f., HAX (1985), S. 62, BURCHERT/HERING/HOFFJAN (1998), S. 246 f., ADAM (2000), S. 120 f., HERING (2017),

S. 139 ff. Insbesondere zu Ganzzahligkeitsproblemen vgl. HAX (1964), S. 442 f. 


\subsubsection{Totalmodell zwischen theoretischer Exaktheit und praktischen Komplexitätsproblemen}

Auf dem unvollkommenen Kapitalmarkt ist somit erforderlich, neben dem Bewertungsobjekt auch Zielsetzung und Entscheidungsfeld einzubeziehen. Ein derartiges Modell, das neben individuellen Konsumentnahmepräferenzen auch andere zur Verfügung stehende Objekte betrachtet, nennt man Totalmodell. ${ }^{108}$ Aus dem simultan ermittelten, optimalen Investitions- und Finanzierungsprogramm geht nicht nur der Grenzpreis hervor, sondern auch die im optimalen Programm vorkommenden Objekte und die aus ihnen hervorgehenden Kalkulationszinsfüße. ${ }^{109}$

Zwar sind Totalmodelle nicht über alle Kritik erhaben. Sie führen im Rahmen ihrer Prämissen zur theoretisch richtigen Lösung, bringen jedoch Komplexitätsprobleme mit sich. ${ }^{110}$ Echte Totalmodelle sind daher ein theoretisches Ideal. ${ }^{111}$ Je realitätsnäher ein Modell formuliert wird, desto unübersichtlicher wird es. In der Realität besteht das Entscheidungsfeld aus einer schier unendlichen Zahl möglicher Investitions- und Finanzierungsobjekte, die auf vielfältige Weise kombiniert werden können. Vor allem bei großen Organisationen stoßen Totalmodelle an die Grenzen der Realisierbarkeit: Die Erfassung des gesamten Entscheidungsfelds mit allen Interdependenzen und Restriktionen führt überall dort zu unvertretbarem Informationsbeschaffungs- und -verarbeitungsaufwand, wo Abstimmung und Koordination eine wichtige Rolle spielen. ${ }^{12}$

Jedoch findet die Bewertung einer Eigentumswohnung durch einen Privatinvestor unter ganz anderen Voraussetzungen statt: ${ }^{113}$ Entscheidungen werden zentral gefällt und das Entscheidungsfeld ist kleiner und überschaubarer - oder kann durch Vereinfachungen derart reduziert werden, daß es problemlos modellierbar ist. ${ }^{114}$ Eine realitätsnahe Modellformulierung in einem zentralen Totalmodell sollte bei Immobilieninvestitionen

${ }^{108}$ Auch zu Folgendem vgl. SCHWEIM (1969), S. 67, HAX (1985), S. 10 f., BRÖSEL/MATSCHKE (2004), S. 65, HERING/SCHNEIDER/TOLL (2010), S. 807.

${ }^{109}$ Totalmodelle sind abzugrenzen von Partialmodellen. Letztere betrachten allein den Zahlungsstrom des Bewertungsobjekts, der mit einem bestimmten, exogen in die Rechnung eingehenden Zinssatz bewertet wird. Das restliche Entscheidungsfeld bleibt außer acht, vgl. FRANKE/LAUX (1968), S. 740 f., OLBRICH (1999), S. 16, BYSIKIEWICZ (2008), S. 140 f., RAPP (2014), S. 170. Auf dem unvollkommenen Kapitalmarkt scheitert deren Anwendbarkeit an der fehlenden Kenntnis eines zur Abzinsung verwendbaren Kalkulationszinsfußes, vgl. COENENBERG (1992), S. 100, ADAM (2000), S. 81, ROLLBERG (2001), S. 62, HERING/OLBRICH (2009), S. 371.

${ }^{110}$ Vgl. zum Folgenden LEUTHIER (1988), S. 123, BURCHERT/HERING/HOFFJAN (1998), S. 247, TOLL (2017), S. 193 f., HERING (2017), S. 218.

${ }^{111}$ Vgl. SCHNEIDER (1992), S. 30, ähnlich auch PAPE (2009), S. 137.

112 Vgl. ADAM (1996), S. 355, BRÖSEL (2017), S. 211, HERING (2017), S. 149 f.

${ }^{113}$ Auch zum Folgenden vgl. TOLL/WALOCHNIK (2013), S. 35.

${ }^{114}$ Zur Komplexitätsreduktion im Rahmen der Unternehmensbewertung vgl. BALLWIESER (1990). 
durch Privatpersonen in den meisten Fällen möglich sein, ohne daß das Bewertungsergebnis zu stark verzerrt wird. ${ }^{115}$

Bevor das Zustands-Grenzpreismodell in Abschnitt 2.2.6 als zielführendes Totalmodell vorgestellt wird, dienen die nachfolgenden Ausführungen dazu, Zielsetzung, Entscheidungsfeld und Bewertungsobjekt näher zu beleuchten.

\subsection{Allgemeine Beschreibung des Bewertungsmodells}

\subsubsection{Zielsystem als Bewertungsausgangspunkt}

Wesentlichen Einfluß auf die Zusammensetzung des optimalen Investitions- und Finanzierungsprogramms übt die subjektive Konsumzielsetzung aus. Diese gibt an, zu welchen Zeitpunkten bzw. in welcher zeitlichen Struktur sich der Investor Entnahmen zu Konsumzwecken wünscht. Da auf dem unvollkommenen Kapitalmarkt unterschiedliche Entnahmemuster nicht ohne weiteres ineinander überführbar sind, wird das optimale Investitions- und Finanzierungsprogramm auch von der Konsumzielsetzung bestimmt. ${ }^{116}$

Aus der rein quantitativen Sichtweise ist eine Totalgewinnmaximierung erstrebenswert. ${ }^{117}$ Sie fordert, über die gesamte Betrachtungsperiode hinweg möglichst hohe Konsumentnahmen vornehmen zu können. Diese Zielsetzung ist jedoch nicht hinreichend operabel. Erst durch Berücksichtigung der subjektiven Konsumpräferenz gelingt es, sie modellmäßig erfaßbar zu machen: Man konkretisiert den Wunsch nach Totalgewinnmaximierung typischerweise als Einkommens- oder Vermögensmaximierung. ${ }^{118}$ Einkommensmaximierung umschreibt das Ziel, bei gegebenen Vermögensentnahmen die Breite eines strukturierbaren Entnahmestroms zu maximieren. Vermögensmaximierung hingegen verkörpert die Zielsetzung, bei gegebenem Einkommen zu einem oder mehreren Zeitpunkten ein größtmögliches Vermögen zu entnehmen.

Für die Investition in eine Eigentumswohnung erscheint die Zielsetzung Einkommensmaximierung besonders passend: Wegen der meist kreditfinanzierten, hohen Kapitalbindung, einhergehend mit ebenfalls sehr hohen Transaktionskosten, kann man in der Regel von einer langfristigen Investitionsdauer ausgehen. Demgegenüber kann der In-

\footnotetext{
${ }^{115}$ Letztlich ist jedes Modell ein Partialmodell, denn ein ,alle betrieblichen Zusammenhänge erschöpfend abbildendes Totalmodell gibt es auf Grund der beschränkten menschlichen Informationsgewinnungs- und -verarbeitungskapazität nicht und wird es auch niemals geben." ROLLBERG (2001), S. 4.

116 Vgl. HERING (2014), S. 26 f., TOLL/HERING (2017), S. 474.

${ }^{117}$ Auch zu Folgendem vgl. KLINGELHÖFER (2006), S. 24, HERING/SCHNEIDER/TOLL (2010), S. 806 f., ähnlich $\operatorname{SIEBEN}(1967)$, S. 127.

${ }^{118}$ Vgl. HERING (2000c), S. 434, TOLL (2011), S. 31 f., JAPES (2011), S. 11 f., HERING (2017), S. 19 ff.
} 
vestor über viele Jahre Mieteinnahmen in gleicher Höhe vereinnahmen. Der gelegentlich für Mehrfamilienhäuser verwendete Begriff ,Zinshaus“"119 verdeutlicht diesen Aspekt. Deswegen wird im Folgenden von der Zielsetzung Einkommensmaximierung ausgegangen.

\subsubsection{Entscheidungsfeld als Bewertungsrahmen}

Unter dem Entscheidungsfeld versteht man die Rahmenbedingungen der Bewertungssituation. Es beinhaltet sämtliche Handlungsoptionen und Restriktionen, die für die Bewertungssituation von Bedeutung sein können. ${ }^{120}$ Dies können z.B. Obergrenzen hinsichtlich der Verfügbarkeit mancher Objekte sein, z.B. Kreditobergrenzen, ebenso wie Nichtnegativitätsbedingungen, Ganzzahligkeitsforderungen aufgrund von Unteilbarkeit oder die jederzeitige Wahrung der Liquidität. Um zu einem brauchbaren Entscheidungswert zu kommen, müssen bspw. die finanziellen Auswirkungen feststehender Einnahmen und Ausgaben sowie bereits im Besitz befindlicher Investitionsobjekte erfaßt werden. Auch ein potentiell vorhandenes Anfangsvermögen übt Einfluß auf die finanzielle Handlungsfähigkeit der Folgeperioden aus. Grundsätzlich ist davon auszugehen, daß die unbegrenzte Möglichkeit zur zinslosen Kassenhaltung besteht. Meist gibt es weitere Objekte zur einperiodigen, verzinslichen ${ }^{121}$ Geldanlage oder Kreditaufnahme. Häufig stehen auch Kredite mit längerer Laufzeit zur Verfügung, ebenso wie alternative Investitionsobjekte. Zu beachten sind auch zwischen den Objekten bestehende Interdependenzen. ${ }^{122}$ Denkbar ist, daß Investitionen sich gegenseitig ausschließen, voneinander abhängen oder bei gemeinsamer Realisierung (positive oder negative) Synergieeffekte erzeugen.

\subsubsection{Eigentumswohnung als Bewertungsobjekt}

\subsubsection{Merkmale von Immobilien}

Nun gilt es, das Bewertungsobjekt zu charakterisieren. Bevor auf die Eigenheiten von Eigentumswohnungen eingegangen wird, ist zunächst erforderlich, die Merkmale der ihnen übergeordneten Klasse der Immobilien herauszustellen: „Mit dem Begriff Immobilie werden umgangssprachlich Grund und Boden, Gebäude sowie deren Kombination

119 WESTERHEIDE (2017), S. 494, ROTTKE/MARKMANN (2017), S. 45, LINDMAYER/DIETZ (2018), S. 41.

120 „Das Entscheidungsfeld beinhaltet sowohl die Menge der möglichen Handlungsalternativen als auch alle für die jeweilige Bewertungssituation relevanten Umweltzustände." ALVANO(1988), S. 109. Zum Inhalt des Entscheidungsfelds vgl. HAX (1964), S. 435 HENSELMANN(1999), S. 28, ROLLBERG (2001), S. 2, MATSCHKE/BRÖSEL (2013), S. 169.

${ }^{121}$ Zumindest vor dem Einmarsch der Negativzinspolitik in die Eurozone war zumeist von der Verfügbarkeit verzinslicher Geldanlageobjekte auszugehen, vgl. dazu auch Abschnitt 2.3.3, insbesondere Fußnote 396.

${ }^{122}$ Vgl. dazu ALBACH (1962), S. 107, HAX (1985), S. 95 ff., GUTENBERG (1987), S. 369 f., BALLWIESER/ HACHMEISTER (2016), S. 14, BITZ/EWERT/TERSTEGE (2018), S. 11 ff. Zur mathematischen Quantifizierung von Interdependenzen vgl. HEIDER (1969), S. $81 \mathrm{ff}$. 
unabhängig von den konkreten Besitz- oder Eigentumsverhältnissen bzw. Verfügungsrechten bezeichnet." ${ }^{123}$ Zur Abgrenzung von anderen Wirtschaftsgütern und Anlageformen werden in der Literatur folgende immobilientypische Merkmale genannt:

- Standortgebundenheit, ${ }^{124}$

- Unikatscharakter bzw. ausgeprägte Heterogenität, ${ }^{125}$

- begrenzte Substituierbarkeit insbesondere der Wohnfunktion, ${ }^{126}$

- hohe Kapitalbindung, ${ }^{127}$ einhergehend mit vergleichsweise schlechter kurzfristiger Liquidierbarkeit ${ }^{128}$ und hohen Transaktionskosten, ${ }^{129}$

- lange Lebens- bzw. Nutzungsdauer ${ }^{130}$ und entsprechend langer Planungshorizont. ${ }^{131}$

Zur Systematisierung von Immobilien hat sich eine Unterteilung nach der Funktion bzw. Nutzungsart etabliert. ${ }^{132}$ Man unterscheidet u.a. zwischen Wohn-, gemischt genutzten und Nichtwohngebäuden. ${ }^{133}$ Die in der vorliegenden Arbeit fokussierten Wohngebäude können auf folgende Weise systematisiert werden:

- Nach der Anzahl an Wohneinheiten mit Zugang zum Treppenhaus unterscheidet man Ein-, Zwei- und Mehrfamilienhäuser. ${ }^{134}$

- Mehrfamilienhäuser können nach der rechtlichen Struktur des Eigentums kategorisiert werden. ${ }^{135}$ So kann das ganze Gebäude einer Person, Personengruppe oder Institution allein gehören. Eine Besonderheit sind darüber hinaus Wohnungseigentümergemeinschaften, bei denen jede Wohneinheit einem anderen Eigentümer gehören kann.

${ }^{123}$ ROPETER (1998), S. 64.

${ }^{124}$ Vgl. GROMER (2012), S. 18 f., SPARS (2017), S. 449, BINCKEBANCK/GÖTZEN (2017), S. 927.

${ }^{125}$ Vgl. OLBRICH (2003), S. 348, TOLL/WALOCHNIK (2013), S. 34, HOMANN (2017), S. 303.

126 Vgl. HAAS (2010), S. 13, ROTTKE/KRAUTZ (2017), S. 783 ff.

127 Vgl. FELDMANN/GERSTNER/HOFMANN/ISENḦ̈FER/SEGERER/V̈̈TH (2016), S. 392, SCḦ̈FERS/HOLZMANN/SCHULTE/LANG/SCHOLZ (2016), S. 483, BRAUER (2019a), S. 8 f.

${ }^{128}$ Vgl. SCHÄFERS/WURSTBAUER (2016), S. 1043, MARKMANN/MARKMANN/ROTTKE (2017), S. 636.

${ }^{129}$ Vgl. REHKUGLER (2011b), S. 249, SCHULZE/STEIN/TIETGEN/MÖLLER (2017), S. 28 ff.

${ }^{130} \mathrm{Vgl}$. KURZROCK (2017a), HERR (2017), S. 434, BRAUER (2019a), S. 9.

${ }^{131}$ Vgl. PFNÜR (2011), S. 98, SCHULTE/SOTELO/ALLENDORF/ROPETER-AHLERS/LANG (2016), S. 589, LEOPOLDSBERGER/THOMAS/NAUBEREIT (2016), S. $448 \mathrm{f}$.

${ }^{132} \mathrm{Vgl}$. REHKUGLER (2011a), S. 5, ARENS (2016), S. 84.

${ }^{133}$ Vgl. GANS (2017), S. 116 f., ROTTKE/EIBEL/KRAUTZ (2017), S. 5. Weitere Unterscheidungsmerkmale sind z.B. Lage, vgl. SCHULTE/HOLZMANN (2005), S. 40, SCHULZE/STEIN/TIETGEN/MÖLLER (2017), S. 133 ff., ERTLE-STRAUB (2019), S. 372, oder Größe und Ausstattung, vgl. FELDMANN/GERSTNER/ HOFMANN/ISENHÄFER/SEGERER/VÄTH (2016), S. 411, SCHARMANSKI/WIENCKE (2017), S. 736 f. Zur Systematisierung von Nichtwohngebäuden vgl. ROPETER (1998), S. 73, BRUNNER (2009), S. 21.

${ }^{134}$ Vgl. KELLER (2013a), S. 36 f., ARENS (2016), S. 85 f., ROTTKE/EIBEL/KRAUTZ (2017), S. 5 ff.

${ }^{135}$ Vgl. RENNERT (2012), S. 76, MUSIELACK (2017), S. 50, DRASDO (2017a), Rn. 104 ff. 
- Aus Anwohnersicht können die in Mehrfamilienhäusern gelegenen Wohnungen entsprechend den Besitzverhältnissen in Miet- und Eigentumswohnungen gegliedert werden. ${ }^{136}$

\subsubsection{Besonderheiten von Eigentumswohnungen}

\subsection{Wohnungseigentum als besondere Form des Eigentums}

Unter einer Wohnungseigentümergemeinschaft (WEG) versteht man die Gemeinschaft der Eigentümer eines Grundstücks. ${ }^{137}$ Diese Rechtsform enthält Komponenten der Bruchteilsgemeinschaft aus $\S \S 741 \mathrm{ff}$. BGB. Die Rechtsfähigkeit der WEG ist auf die Verwaltung des gemeinschaftlichen Eigentums beschränkt. ${ }^{138}$ Unter dieser sogenannten Teilrechtsfähigkeit versteht man die „Fähigkeit, als Rechtssubjekt am Rechtsverkehr aktiv teilnehmen zu können, Träger von Rechten und Pflichten zu sein, im gerichtlichen Verfahren klagen und verklagt werden zu können." ${ }^{\text {"139 }}$

Die Eigentümergemeinschaft besitzt drei Organe: ${ }^{140}$ Der Verwalter ist der gesetzliche Vertreter der WEG im Außenverhältnis. ${ }^{141}$ Er selbst ist mit relativ wenigen Befugnissen ausgestattet; Entscheidungen ab einer gewissen Tragweite müssen auf der Eigentümerversammlung entschieden werden. ${ }^{142}$ Sie ist das konstitutive Organ der WEG. Drittens gibt es einen aus drei Eigentümern bestehenden Verwaltungsbeirat. ${ }^{143}$ Dieser soll den Verwalter unterstützen und die Jahresabrechnung überprüfen. ${ }^{144}$ Mit dieser vagen Formulierung ist alles und auch nichts gesagt, die Interpretation erfolgte vielmehr durch

\footnotetext{
${ }^{136} \mathrm{Vgl}$. SCHULZE/STEIN/TIETGEN/MÖLLER (2017), S. 120 f., ROTTKE (2017c), S. 148 f.

${ }^{137}$ Auch zum Folgenden vgl. MUSIELACK (2017), S. 50 ff., FESSELMANN/KOBABE/SCHWENZFEIER/ SIEGEMUND (2018), S. 52. Die Rechtsgrundlagen sind im Wohnungseigentumsgesetz (WoEigG) geregelt. Diese Rechtskonstruktion wurde in Deutschland 1951 geschaffen, um möglichst vielen Menschen in der Nachkriegszeit den Zugang zu bezahlbarem Wohnraum zu ermöglichen. Zu den Anfängen des Wohnungseigentums vgl. VON HAUFF (2017a), S. 23, MUSIELACK (2017), S. 49, ROTTKE/MARKMANN (2017), S. 60, DRASDO (2017a), Rn. 9.
}

${ }^{138}$ Vgl. SCHNEIDER (2017), Rn. 4.

139 STÜRZER/KOCH/HOPFENSPERGER/STERNS/STERNS-KOLBECK/ZIEGELMAYER (2016), S. 241. „Vor der Entscheidung des BGH (2.6.2005, V ZB 32/05) zur Teilrechtsfähigkeit der Wohnungseigentümergemeinschaft und ihrer gesetzlichen Verankerung im Rahmen der WEG-Reform 2007 waren die Wohnungseigentümer persönlich Träger sämtlicher Rechte und Pflichten, die sich aus der Verwaltung des gemeinschaftlichen Vermögens einer Wohnungseigentümergemeinschaft ergaben." ebd. Der BGH ,postulierte den Grundsatz, dass die Gemeinschaft der Wohnungseigentümer rechtsfähig sei, soweit sie bei der Verwaltung des gemeinschaftlichen Eigentums am Rechtsverkehr teilnehme.“ MUSIELACK (2017), S. 51. Zur Teilrechtsfähigkeit vgl. auch DRASDO (2017a), Rn. 45 f., SUILMANN (2018a), Rn. 208 f.

\footnotetext{
${ }^{140}$ Vgl. VON HAUFF (2017d), S. 201 f., ELZER (2019), Rn. 144.

${ }^{141}$ Vgl. SAUREN (2014e), Rn. 79, MUSIELACK (2017), S. 82, BAER (2019), Rn. 163 ff.

${ }^{142} \mathrm{Vgl}$. dazu Gliederungspunkt 2.2.3.3.2.2.

${ }^{143}$ Vgl. zu diesem DRASDO (2012), SAUREN (2014f), MUNZIG (2019).

${ }^{144}$ Vgl. § 29 WoEigG.
} 
die Rechtsprechung: Der Beirat hat keine Entscheidungsbefugnis, sondern dient als Bindeglied zwischen Verwalter und Eigentümern; im Mittelpunkt steht oft die Kommunikation mit den Wohnungseigentümern. ${ }^{145}$

Eine WEG entsteht durch die notarielle Teilungserklärung. Der bisherige Alleineigentümer erklärt die Teilung der Immobilie in Sondereigentumseinheiten: ${ }^{146}$ Obwohl es sich um ein Grundstück mit einem ${ }^{147}$ Gebäude handelt, gibt es ebenso viele Grundbücher wie es Sondereigentumseinheiten gibt. ${ }^{148}$ SAUREN nennt Wohnungseigentum gar eine neue Eigentumsart. ${ }^{149}$ Diese ermöglicht, daß unterschiedliche Teile desselben Gebäudes verschiedenen Eigentümern gehören können. ${ }^{150}$ Hingegen ist es nach den gewöhnlichen Regelungen des BGB zum Gesamteigentum zwar möglich, daß mehrere Eigentümer gemeinsam ein ganzes Haus besitzen. Da es sich aber um eine ideelle Teilung handelt, ${ }^{151}$ kann niemand sagen, wem welche Wohnung gehört. ${ }^{152}$

Voraussetzung der Teilungserklärung ist die Abgeschlossenheitsbescheinigung, ${ }^{153}$ mit welcher die zuständige Behörde bestätigt, daß die Sondereigentumseinheiten jeweils in sich abgeschlossen sind. Vereinfacht gesagt trennt eine Tür die Wohnung vom Treppenhaus. Und so differenziert das Wohnungseigentumsrecht zwischen Sonder- und Gemeinschaftseigentum. ${ }^{154}$ Sondereigentum entsteht durch sachliche Zuordnung von ex-

${ }^{145}$ In der Praxis wird die Weisungsbefugnis - vor allem von den Beiratsmitgliedern selbst - teils massiv überschätzt. Dazu äußert sich VON HAUFF lobenswert deutlich: „Es kann nicht sein, dass der eine (Verwalter) haftet und der andere (Beirat) entscheidet. Wer als Verwalter dieser Gefahr erliegt, wird zwar kurzfristig mit Lob in der nächsten Eigentümerversammlung rechnen können, aber langfristig sein Amt in Gefahr bringen. Nur die Eigentümerversammlung kann den Verwalter rechtswirksam beauftragen, niemand anderes." VON HAUFF (2017d), S. 232.

${ }^{146}$ Vgl. SCHNEIDER (2017), Rn. 42, ARMBRÜSTER (2018c), Rn. 3. Zu Form und Inhalt der Teilungserklärung vgl. MÜLLER (2015a), Rn. 41. Wenn das Mehrfamilienhaus bislang mehreren Eigentümern gehörte, tritt der Teilungsvertrag an die Stelle der Teilungserklärung. Zu diesem vgl. SCHWERING (2019), S. 289.

${ }^{147}$ Die Begründung einer WEG an einem Grundstück mit mehreren Gebäuden ist ebenfalls zulässig, nicht jedoch, wenn mehrere Grundstücke involviert sind, vgl. § 1 Abs. 4 WoEigG. Zu Wohnungseigentum bei Mehrhausanlagen vgl. SCHMIDT (1989), SUILMANN (2018a), Rn. 26 ff.

${ }^{148}$ Vgl. SCHNEIDER (2017), Rn. 120 f., MÜTHLEIN/HOFFMANN (2017), S. 10 ff.

$149 \operatorname{SAUREN}(2014 \mathrm{a}), \mathrm{Rn} .2$.

${ }^{150}$ Vgl. STEINER (2009), S. 230, STÜRZER/KOCH/HOPFENSPERGER/STERNS/STERNS-KOLBECK/ZIEGELMAYER (2016), S. 17 ff. Mit dem Wohnungseigentumsgesetz ermöglicht der Gesetzgeber ,in Abweichung von der in den $\S \S 93,94$ BGB enthaltenen Regel, nach der Eigentum an Gebäudeteilen und Räumen allein nicht zulässig ist, die Bildung von Sondereigentum an bestimmten Räumen eines Gebäudes." MUSIELACK (2017), S. 49.

${ }^{151}$ Vgl. § 93 ff. BGB, siehe auch MÜLLER (2015a), Rn. 1, DRASDO (2017a), Rn. 18.

$152 \mathrm{Vgl}$. MUSIELACK (2017), S. 50.

${ }^{153}$ Vgl. § 3 Abs. 2 WoEigG. Vgl. dazu auch HÜGEL/ELZER (2018a), Rn. 26.

${ }^{154}$ Drittens gibt es Verwaltungsvermögen, z.B. die Instandhaltungsrücklage. In Abgrenzung zum Gemeinschaftseigentum, welches allen Eigentümern anteilig gehört, steht das Verwaltungsvermögen 
plizit genannten Räumen bzw. Gebäudeteilen durch ausdrückliche Nennung in der Teilungserklärung. Alles andere verbleibt im Gemeinschaftseigentum, welches - wie bei der BGB-Gemeinschaft - allen Eigentümern gemeinschaftlich gehört. ${ }^{155}$ Das Grundstück ${ }^{156}$ sowie Teile des Gebäudes, die für dessen Bestand oder Sicherheit ${ }^{157}$ oder äußeres Erscheinungsbild ${ }^{158}$ bedeutsam sind oder dem gemeinschaftlichen Gebrauch ${ }^{159}$ dienen, stehen zwingend im Gemeinschaftseigentum. ${ }^{160}$ Häufig genannte Beispiele sind tragende Mauern, Fassaden (-abschnitte) und Fenster.

Einem Wohnungseigentümer ohne juristische Vorbildung ist nur schwer zu erklären, daß er keine eigenständige Wohnung, sondern nur das Sondereigentum an bestimmten Räumlichkeiten gekauft hat. ${ }^{161}$ Sogar die Wohnungsabschlußtür gehört zum Gemeinschaftseigentum, weil sie das einheitliche Erscheinungsbild des Treppenhauses prägt und dazu dient, Sonder- und Gemeinschaftseigentum voneinander abzugrenzen. ${ }^{162}$ Der ideelle Miteigentumsanteil (MEA) am Gemeinschaftseigentum, welcher untrennbar mit dem Sondereigentum verbunden ist und bestimmte Nutzungsrechte mit sich bringt, verkörpert die Brücke zwischen Sonder- und Gemeinschaftseigentum: ${ }^{163}$ Jeder Wohnungseigentümer ist gleichzeitig Anteilseigner am gemeinschaftlichen Eigentum.

Sondereigentum tritt wiederum in verschiedenen Erscheinungsformen auf: Handelt es sich um Wohnräume, spricht man von Wohnungseigentum, andernfalls von Teileigentum (z.B. bei Praxis-, Büro-, Kellerräumen oder Garagen). ${ }^{164}$ Einen Sonderfall stellen

im Eigentum der WEG, vgl. SCHNEIDER (2017), Rn. 29 f., HÜGEL (2019a), Rn. 17. Bei der Abgrenzung ergeben sich in der Praxis teils erhebliche Probleme. Die Rechtsprechung hat zahllose, teils skurrile Beispiele hinsichtlich der Zuordnung zu Sonder- (SE) oder Gemeinschaftseigentum (GE) bzw. Verwaltungsvermögen (VV) hervorgebracht, z.B. Absperrventile (GE), Balkontrennmauern (GE), Briefkästen (GE), Früchte eines Obstbaums (VV), Gartenwasserhähne (GE), Isolierschichten, (GE), Jalousien (GE, Gurte hingegen SE), Schneeschippe (nicht GE, sondern VV), Steckdosen (im Bereich von Außenbalkonen: SE), Wohnungstüren (GE), Verwaltungsunterlagen (VV), Wasserzähler (GE), vgl. SAUREN (2014a), Rn. 10, RAMPP (2017), Rn. 66, ARMBRÜSTER (2018b), Rn. 49 ff.

${ }^{155}$ Vgl. RAMPP (2017), Rn. 54, ARMBRÜSTER (2018a), Rn. 52 ff., SCHWERING (2019), S. 292 f.

${ }^{156}$ Vgl. § 1 Abs. 5 WoEigG.

${ }^{157}$ Man denke z.B. an tragende Wände innerhalb einer Wohnung. Die Gebäudesicherheit kann gefährdet sein durch „Einsturz, Brand, Regenwasser“. RAMPP (2017), Rn. 47.

${ }^{158}$ Bspw. Fassade, Fenster oder Terrassen, vgl. SAUREN (2014a), Rn. 10, HÜGEL (2019a), Rn. 12.

${ }^{159}$ Zum Beispiel Aufzug, Hausflur oder Treppenhaus, vgl. MÜLLER (2015b), Rn. 13.

${ }^{160}$ Vgl. § 5 Abs. 2 WoEigG.

${ }^{161}$ Auch zum Folgenden vgl. STÜRZER/KOCH/HOPFENSPERGER/STERNS/STERNS-KOLBECK/ZIEGELMAYER (2016), S. 19, MUSIELACK (2017), S. 50, SCHWERING (2019), S. 293.

${ }^{162} \mathrm{Vgl}$. MÜLLER (2015b), Rn. 24, ARMBRÜSTER (2018b), Rn. 124.

${ }^{163}$ Vgl. § 1 Abs. 2 f., § 6 Abs. 1 WoEigG. Auch zum Folgenden vgl. FROESE (2015), S. 24 f., SCHNEIDER (2017), Rn. 1 ff.

${ }^{164}$ Vgl. § 1 WoEigG, siehe dazu B̈̈R (2017), S. 15, MÜTHLEIN/HOFFMANN (2017), S. 10 ff. 
Sondernutzungsrechte dar: Weil Grundstücksteile wie bspw. Gartenflächen nicht räumlich abgeschlossen sein können, ist die Begründung von Teileigentum unmöglich, ${ }^{165}$ wohl aber die Einräumung eines ausschließlichen Nutzungsrechts. ${ }^{166}$ Tabelle 3 faßt zusammen, wonach sich die Zuordnung zu Sonder- und Gemeinschaftseigentum richtet.

\begin{tabular}{|c|c|}
\hline Definition & Beispiel \\
\hline $\begin{array}{l}\text { Sondereigentum: } \\
\text { - Ausdrücklich bestimmte, nach außen } \\
\text { hin abgeschlossene Wohnräume } \\
\text { (Wohnungseigentum) oder nicht zu } \\
\text { Wohnzwecken dienende Räume } \\
\text { (Teileigentum) und } \\
\text { - zu diesen Räumen gehörende Gebäu- } \\
\text { deteile, die verändert, beseitigt oder } \\
\text { eingefügt werden können, ohne daß } \\
\text { dadurch anderes Sondereigentum be- } \\
\text { einträchtigt oder gemeinschaftliches } \\
\text { Eigentum oder die äußere Gestaltung } \\
\text { des Gebäudes verändert werden. }\end{array}$ & $\begin{array}{l}\text { Sondereigentum: } \\
\text { - }, \text {,Die im Grundriß (Anlage } 1 \text { zur Tei- } \\
\text { lungserklärung) mit Ziffer } 7 \text { bezeich- } \\
\text { neten Wohnräume im } 2 . O G \text { li. (72,56 } \\
\text { qm) ...“, einschließlich } \\
\text { - } \text { nichttragende Zwischenwände, } \\
\text { - Innentüren, Heizkörper, } \\
\text { - Decken-, Wand- und Fußbodenbelag, } \\
\text { - Versorgungsleitungen ab dem Zwi- } \\
\text { schenzähler bzw. ab dem Abzweig } \\
\text { vom Hauptstrang. }\end{array}$ \\
\hline $\begin{array}{l}\text { Gemeinschaftseigentum: } \\
\text { - Grundstück und } \\
\text { - Gebäudeteile, Anlagen und Einrich- } \\
\text { tungen, } \\
\text { ○ die dem gemeinschaftlichen Ge- } \\
\text { brauch der Wohnungseigentümer } \\
\text { dienen, auch wenn sie sich im } \\
\text { Sondereigentum befinden, } \\
\text { die für Bestand oder Sicherheit des } \\
\text { Gebäudes erforderlich sind oder } \\
\text { die nicht zu Sondereigentum er- } \\
\text { klärt wurden. }\end{array}$ & $\begin{array}{l}\text { Gemeinschaftseigentum: } \\
\text { - } \text { Dach, } \\
\text { - } \text { Treppenhaus, } \\
\text { - } \text { Gartralheizung, } \\
\text { - Fassade, } \\
\text { - (Außen-) Fenster, } \\
\text { - (tragende) Wände, } \\
\text { - Wohnungstüren. }\end{array}$ \\
\hline
\end{tabular}

Tabelle 3: Abgrenzung zwischen Sonder- und Gemeinschaftseigentum

${ }^{165}$ Allerdings sieht $\S 3$ Abs. 2 WoEigG eine Ausnahme vor: „Garagenstellplätze gelten als abgeschlossene Räume, wenn ihre Flächen durch dauerhafte Markierungen ersichtlich sind.“

${ }^{166} \mathrm{Vgl}$. STÜRZER/KOCH/HOPFENSPERGER/STERNS/STERNS-KOLBECK/ZIEGELMAYER (2016), S. 43 f., SCHULZE/STEIN/TIETGEN/MÖLLER (2017), S. 119, SCHWERING (2019), S. 294 f. Auch (Tiefgaragen-) Stellplätze gelten nach $\S 3$ Abs. 2 WoEigG als ,,abgeschlossene Räume, wenn ihre Flächen durch dauerhafte Markierungen ersichtlich sind.“ 


\subsection{Position des vermietenden Wohnungseigentümers zwischen Miet- und Wohnungseigentumsrecht}

Ein vermietender Wohnungseigentümer befindet sich zwangsläufig in zwei divergierenden Rechtssphären: ${ }^{167}$ Im Verhältnis zur WEG ist er einerseits stimmberechtigter Mitgesellschafter. Sein Einfluß kann entweder mit einem faktisch stimmrechtslosen Kleinaktionär vergleichbar sein, andererseits ist es möglich, durch Kauf mehrerer Wohnungen mehr als die Hälfte der Stimmrechte zu erwerben. ${ }^{168}$ Aus dem Gesellschafterverhältnis erwachsen u.a. folgende Rechte und Pflichten:

- Mitbenutzungsrecht am Gemeinschaftseigentum (Heizung, Treppenhaus,...), ${ }^{169}$

- Pflicht zur anteiligen Kostentragung, ${ }^{170}$

- Stimm- und Mitspracherecht in der Eigentümerversammlung. ${ }^{171}$

Im Verhältnis zum Mieter ist er andererseits dessen Vertragspartner: Jeder Eigentümer kann mit seinem Sondereigentum ,nach Belieben verfahren“, sofern „,nicht das Gesetz oder Rechte Dritter entgegenstehen“, sagt $\S 13$ Abs. 1 des WoEigG. Mietersuche und Gestaltung des Mietvertrags fallen grundsätzlich unter die Entscheidungshoheit des Vermieters, schließlich besteht Vertragsfreiheit. Aus dem Mietvertrag erwachsen völlig andere Rechte und Pflichten, darunter:

${ }^{167}$ Einen Überblick über diesen Problemkreis geben SUILMANN (2017a), LEHMANN-RICHTER (2019), SUILMANN (2019). Zu Folgendem vgl. STÜRZER/KOCH/HOPFENSPERGER/STERNS/STERNS-KOLBECK/ZIEGELMAYER (2016), S. 295 ff., VON HAUFF (2017a), S. 23 f.

${ }^{168}$ Dies ist jedoch nur dann möglich, wenn die Teilungserklärung eine Stimmrechtsregelung enthält, z.B. ein Stimmrecht nach Wohneinheiten oder nach Miteigentumsanteilen. Fehlt eine Vereinbarung, gilt das gesetzliche Kopfprinzip: Hier hat jeder Eigentümer eine Stimme, egal wie viele Wohnungen er besitzt, vgl. RÜSCHER (2017b), Rn. 18, MERLE (2018c), Rn. 29 f., BARTHOLOME (2019a), Rn. 9 ff.

${ }^{169}$ Vgl. SUILMANN (2018c), Rn. 37 ff., FESSELMANN/KOBABE/SCHWENZFEIER/SIEGEMUND (2018), S. 53, $H U ̈ G E L$ (2019a), Rn. 13 ff.

${ }^{170}$ Die von den Eigentümern zu tragenden Kosten gehen weit über die mietrechtlichen Betriebskosten hinaus und umfassen beispielsweise Kosten der Instandhaltung und Instandsetzung des Gemeinschaftseigentums, der Verwaltung oder von Rechtsstreitigkeiten. Vgl. zur Kostentragung der Wohnungseigentümer WANDERER (2017b), Rn. 30 ff., RAMPP (2017), Rn. 45, BECKER (2018a), Rn. 21 ff.

${ }^{171}$ Vgl. zur Eigentümerversammlung RÜSCHER (2017a), MERLE (2018b), insbesondere zum Stimmrecht vgl. SAUREN (2014d), Rn. 4 ff., VON HAUFF (2017d), S. 218, ELZER (2018a), Rn. 308 ff. Ein potentieller Nachteil von Eigentumswohnungen kann das allgegenwärtige Mitbestimmungsrecht der Nachbarn sein. Es wird ,immer erst dann zum Problem, wenn die Menschen nicht in der Lage sind, ihr Gemeinschaftsverhältnis den demokratischen Gepflogenheiten entsprechend auszugestalten bzw. sich nach den demokratischen und menschlichen Spielregeln zu verhalten. Solche Verhaltensstörungen ergeben sich aber nicht nur im Wohnungseigentum. Sie sind auch [...] bei Reihenhäusern, aber auch bei freistehenden Einfamilienhäusern nicht auszuschließen." DRASDO (2017a), Rn. 115. Zu problematischen Eigentümern äußert sich auch SCHWERING (2019), S. 292: „Die Folgen mangelnder Sorgfalt [bei Erstellung in der Teilungserklärung] zeigen sich oft erst nach Jahren, wenn steigende Kosten, anfallende Reparaturen, Eigentümerwechsel oder querulatorische Beteiligte die Teilungserklärung bezweifeln und gerichtlich überprüfen lassen." Doch war bereits der Vorläufer von Eigentumswohnungen, ,das sogenannte Stockwerkseigentum, in Südwestdeutschland als ,Streithäusle“ verschrien.“ VON HAUFF (2017a), S. 23. 
- Anspruch auf Zahlung von Miete und Betriebskosten, ${ }^{172}$

- Möglichkeit der Mieterhöhung, ${ }^{173}$

- Pflicht, eine jährliche Abrechnung über die Betriebskosten zu erstellen, ${ }^{174}$

- Sorgfaltspflichten, z.B. Aufrechterhaltung des vertragsgemäßen Zustands. ${ }^{175}$

Aus diesem „doppelten Pflichtenkreis““176 können sich Kollisionen ergeben: Der Mieter wohnt zwar im Gebäude, ist aber in keiner Weise an Beschlüsse bzw. Vereinbarungen der WEG gebunden - z.B. entfaltet eine Hausordnung ${ }^{177}$ oder ein Hundehaltungsverbot $^{178}$ nur innerhalb der Eigentümergemeinschaft Bindungswirkung. Die WEG kann deren Einhaltung nur vom vermietenden Sondereigentümer, nicht aber von seinem Mieter fordern. Häufig kommt es zu Konflikten, wenn der Vermieter noch keine Betriebskostenabrechnung erstellen kann, weil er die WEG-Jahresabrechnung noch nicht erhalten hat. ${ }^{179}$ Auch Kostenverteilschlüssel und andere Modalitäten der Abrechnung können im Mietvertrag völlig anders geregelt sein als in der Teilungserklärung. ${ }^{180}$

Probleme sind ferner bei Mängeln im Gemeinschaftseigentum vorstellbar, weil dessen Mitbenutzungsrecht in den meisten Fällen zum vertragsgemäßen Gebrauch der Mietsache gehört. ${ }^{181}$ SUILMANN nennt in diesem Kontext beispielsweise den Ausfall eines Personenaufzugs oder eine durch Vandalismus zerstörte Haustür. ${ }^{182}$ Während der Vermieter Schäden innerhalb des Sondereigentums meistens kurzfristig reparieren kann, ist die WEG im Bereich des Gemeinschaftseigentums zuständig. ${ }^{183}$ Ungeachtet dessen stehen dem Mieter möglicherweise bereits Minderungsrechte zu, während der Vermieter sich zwar unverzüglich um eine Lösung bemüht, aber allein keine Abhilfe schaffen kann. ${ }^{184}$

172 Vgl. VON BRESSENSDORF (2016), S. 246 f., ZEHELEIN (2019), Rn. 508 ff., HUBER (2019), S. 213.

${ }^{173}$ Vgl. BÖRSTINGHAUS (2017c), BUSCH (2017a), S. 691, SCHÜLLER (2019), Rn. 3 ff.

${ }^{174}$ Vgl. zu Betriebskosten und deren Abrechnung STREYL (2017), Rn. 110 ff., WEBER (2019), Rn. 8, WIEDERHOLD (2019), Rn. 86 ff. Grundsätzlich besteht die Möglichkeit, eine Pauschale zu erheben. Dann kann der Vermieter, unabhängig von den tatsächlich angefallenen Kosten, keinerlei Nachzahlung mehr fordern, vgl. LANGENBERG (2017a), Rn. 247 ff., PFEIFER (2019a), Rn. $1182 \mathrm{ff}$.

175 Vgl. LEOPOLDSBERGER/THOMAS/NAUBEREIT (2016), S. 445, BLANK (2017a), Rn. 345 ff.

176 SUILMANN (2018b), Rn. 41.

${ }^{177}$ Hierzu vgl. SCHMID (2013), MÜLLER (2015c), Rn. 250 ff., FRITSCH (2018), Rn. 557 f.

${ }^{178}$ Vgl. dazu BLANK (2007), SUILMANN (2017a), Rn. 24.

${ }^{179}$ Vgl. zum Folgenden MÜLLER (2015e), Rn. 255, FRITZ(2018), S. 92.

${ }^{180}$ Hierzu vgl. LANGENBERG (2017a), Rn. 313 ff., BECKER (2018c), Rn. 144 f.

${ }^{181}$ Vgl. HÄUBLEIN (2016a), Rn. 70, STÜRZER/KOCH/HOPFENSPERGER/STERNS/STERNS-KOLBECK/ZIEGELMAYER (2016), S. 28 f., SCHMIDT (2019), S. 305 ff., WIEGNER (2019), Rn. 4 f.

${ }^{182}$ Auch zum Folgenden vgl. SUILMANN (2017b), Rn. 1 ff.

${ }^{183}$ Vgl. ENGELHARDT (2017a), Rn. 30, MERLE (2018a), Rn. 131 f., BECKER (2018b), Rn. 62.

${ }^{184} \mathrm{Vgl}$. dazu BUB (2009), S. 245 ff., STÜRZER/KOCH/HOPFENSPERGER/STERNS/STERNS-KOLBECK/ ZIEGELMAYER (2016), S. 305 f., SUILMANN (2017b), Rn. 10 f., SUILMANN (2019). Beispielhaft zur Haftung der Wohnungseigentümer untereinander bei zögerlicher Beschlußumsetzung vgl. JACOBY (2017). 
Selbst wenn auch der WEG-Verwalter an einer Lösung interessiert ist, kann viel Zeit verstreichen. Er hat kaum Entscheidungsspielraum, die Willensbildung einer WEG geschieht allein durch Beschlußfassung auf der Eigentümerversammlung. ${ }^{185} \mathrm{Nicht}$ zu unterschätzen sind Blockadehaltungen von Miteigentümern, die notwendige Beschlüsse verhindern, sei es aus Unwissen, Übereifer, Gleichgültigkeit oder Geldnot. ${ }^{186}$ Selbst bei kooperativen Miteigentümern können Formalitäten wie die Einladungsfrist eine schnelle Lösung vereiteln. ${ }^{187}$ Solche Merkmale sind zwingend zu berücksichtigen, weil sie den finanziellen Zukunftserfolg des Bewertungsobjekts beeinflussen können.

\subsection{Merkmale von Eigentumswohnungen}

Tabelle 4 faßt Merkmale zur Systematisierung von Eigentumswohnungen zusammen, die neben immobilientypischen Kriterien auch Verhältnisse innerhalb der WEG berücksichtigen, bspw. die stimmrechtsrelevante Eigentümerstruktur. ${ }^{188}$

${ }^{185}$ Vgl. BAER (2019), Rn. 28, HERMANN (2019a), Rn. 8.

${ }^{186}$ Wenn die Eigentümer weder Notwendigkeit noch Kosten einer Reparaturmaßnahme richtig einordnen können, kommt es in der Praxis oft dazu, daß der Verwalter aufgefordert wird, zunächst drei Vergleichsangebote einzuholen. Dies, ,ist zwar ein billiger, aber kein preisgünstiger Weg. Nicht nur kann es sein, dass Angebote aus diesem Bereich nicht vergleichbar sind, weil die unterschiedlichen Betriebe unterschiedliche Leistungen anbieten. Die wesentlichste Gefahr und das höchste Restrisiko liegen in nicht durchgeführten Untersuchungen. Dieses Manko tritt in der Regel bei der Ausführung der Leistungen zutage, weil unabsehbare Zusatz- und Nachtragsangebote den vorgesehenen Leistungsrahmen mit der Begründung sprengen, dies sei vorher nicht erkennbar gewesen." MATHAR (2017), S. 260. Außerdem verkennen viele Eigentümer, daß sich gute Handwerker vor allem bei Nachfrageüberhang nicht darauf einlassen. Schließlich wissen sie, daß der Anbieter mit dem niedrigsten Preis gewinnen wird: „Wo noch Handwerker und Hausmeister finden? Die alten gehen in den Ruhestand, von unten kommt (fast) nichts nach. [...] Für die Immobilienbranche könnte sich der Handwerkermangel zur Achillesferse entwickeln“, verdeutlicht UNTERREINER (2018), S. 8. Die richtige Vorgehensweise wäre die Hinzuziehung eines Architekten, denn die „Verfahrensweise, Leistungen ohne vorhergehende Untersuchungen durchführen zu lassen, widerspricht dem Sinne nach bereits den Regeln, die auch im normalen täglichen Leben eingehalten werden. Kein vernünftiger Mensch stimmt einer Operation bei einem Arzt zu, wenn nicht vorher eine Untersuchung erfolgt ist", warnt MATHAR (2017), S. 260. Ähnlich auch WINTZER (2015), S. 19 f.

${ }^{187} \mathrm{Zu}$ Formalitäten der Einladung zur Eigentümerversammlung vgl. MÜLLER (2015d), S. 45 ff., MUSIELACK (2017), S. 85 f.

188 Zur Lage vgl. FELDMANN/GERSTNER/HOFMANN/ISENHÄFER/SEGERER/VÄTH (2016), S. 391, ERTLESTRAUB (2019), S. 372. Zum städteplanerischen Beispiel „Chorweiler als größte Plattenbausiedlung Nordrhein-Westfalens“ vgl. ELLERBROCK (2014), S. 65 f. Zum Gebäudealter vgl. HENGER (2014), S. 244, zu diesem in Bezug auf Bauschäden vgl. MÜLLER (2009), S. 289. Zum Sanierungsbedarf des Sondereigentums vgl. HERR (2017), S. 434, PFLÜGL (2017e), Rn. 10, SCHULZE/STEIN/TIETGEN/ MÖLLER (2017), S. 125. Zu Leerstand als Allokationsergebnis vgl. SPARS (2017), S. 449. Zum Sanierungsbedarf des Gemeinschaftseigentums vgl. VON HAUFF (2017b), S. 46 f., SCHARMANSKI/ WIENCKE (2017), S. 736, KURZROCK (2017a), S. 437. Zur Wartungsintensität vgl. SCHÄFERS/WURSTBAUER (2016), S. 1041 f. Zur immer stärker zunehmenden Gebäudetechnik bei Neubauten vgl. FELDMANN/GERSTNER/HOFMANN/ISENHÄFER/SEGERER/VÄTH (2016), S. 411. Zur Vermietung von Gemeinschaftsflächen an Sondereigentümer vgl. GROßE (2017), S. 504. 


\begin{tabular}{|c|c|}
\hline Merkmal & Mögliche Merkmalsausprägungen \\
\hline Lage & $\begin{array}{l}\text { - Großstadt, Ballungsraum } \\
\text { - Vorort, Großraum einer Stadt } \\
\text { - Ländlich, abgeschieden }\end{array}$ \\
\hline Gebäudedaten & $\begin{array}{l}\text { - Alter: Neubau oder Bestandsgebäude, Baujahr } \\
\text { - Anzahl Wohn- bzw. Teileigentumseinheiten } \\
\text { - Gesamtwohnfläche und Grundstücksfläche }\end{array}$ \\
\hline Wohnungsdaten & $\begin{array}{l}\text { - Wohnfläche, Lage im Gebäude, Stockwerk } \\
\text { - Miteigentumsanteil am Grundstück }\end{array}$ \\
\hline $\begin{array}{l}\text { Nutzungsart der } \\
\text { Wohnung }\end{array}$ & $\begin{array}{l}\text { - Wohnungseigentum: Wohneinheit } \\
\text { - Teileigentum: Praxis, Gewerbeeinheit }\end{array}$ \\
\hline $\begin{array}{l}\text { Sanierungsbedarf } \\
\text { der Wohnung }\end{array}$ & $\begin{array}{l}\text { - } \text { Bezugsfertig } \\
\text { - Renovierungsbedürftig } \\
\text { - Sanierungsbedürftig } \\
\end{array}$ \\
\hline $\begin{array}{l}\text { Derzeitige Nutzung } \\
\text { der Wohnung }\end{array}$ & $\begin{array}{l}\text { - Selbstgenutzt } \\
\text { - Vermietet } \\
\text { - Leerstehend }\end{array}$ \\
\hline \begin{tabular}{|l|} 
Eigentümerstruktur, \\
Stimmrecht
\end{tabular} & $\begin{array}{l}\text { - Viele unterschiedliche Eigentümer } \\
\text { - Ein Mehrheitseigentümer }\end{array}$ \\
\hline $\begin{array}{l}\text { Nutzungsart des } \\
\text { Gebäudes }\end{array}$ & $\begin{array}{l}\text { - Reines Wohngebäude } \\
\text { - Gemischte Nutzung (z.B. Ladenzeile im EG) } \\
\text { - Gewerbeimmobilie } \\
\text { - Ferienhausanlage, Hotel, Altenwohnheim } \\
\end{array}$ \\
\hline \begin{tabular}{|l|} 
Sanierungsbedarf, \\
Wartungsintensität \\
des Gebäudes
\end{tabular} & $\begin{array}{ll}\text { - } & \text { Kein/mittelmäßiger/hoher Instandhaltungsbedarf } \\
\text { - } & \text { Keine/mittelmäßig/viele wartungsbedürftige Einrichtungen } \\
& \text { (z.B. Sprinkleranlage, Tiefgaragenentlüftung, Aufzug) }\end{array}$ \\
\hline $\begin{array}{l}\text { Besonderheiten der } \\
\text { WEG }\end{array}$ & $\begin{array}{l}\text { - Mehrhausanlage mit Untergemeinschaften } \\
\text { - } \quad \text { Vermietetes Gemeinschaftseigentum (z.B. Anbau, Terrasse) }\end{array}$ \\
\hline
\end{tabular}

Tabelle 4: Merkmale von Eigentumswohnungen

\subsubsection{Prognose des Zahlungsstroms von Eigentumswohnungen}

\subsection{Zahlungen im Investitionszeitpunkt}

Um die Eigentumswohnung als mathematisch greifbares Bewertungsobjekt abzubilden, muß ihr Zahlungsstrom prognostiziert werden. ${ }^{189}$ Dieser ist vielschichtig und besteht aus heterogenen Eingangsdaten. Um diese sinnvoll zu systematisieren, empfiehlt sich deren Einordnung nach der Zeit, wobei eine Differenzierung zwischen Investitionszeitpunkt

${ }^{189} \mathrm{Zu}$ Analyseschritten bei der Bestimmung von Zahlungsströmen vgl. VON AHSEN/DE WITT (2009), S. 139 ff., BALLWIESER/HACHMEISTER (2016), S. 48 ff. BRÖSEL und DECHANT zeigen beispielhaft zehn Schritte zur Schätzung der Zahlungsströme von Telekommunikationsunternehmungen, vgl. BRÖSEL/DECHANT (2003), S. 146 ff. Zur Nutzung von Prognoseverfahren vgl. BRETZKE (1977). „Nur deshalb, weil wir nicht in die Zukunft sehen können und weil wir das für Zukunftsschätzungen nötige Material aus der Vergangenheit gewinnen müssen, hat das Vergangene für unsere Schätzungen Interesse.“ SCHMALENBACH (1966), S. 36. 
$(\mathrm{t}=0)$, Ende des Planungshorizonts $(\mathrm{t}=\mathrm{n})$ und dazwischen liegender Vermietungsdauer $(\mathrm{t}=1$ bis $\mathrm{t}=\mathrm{n}-1)$ vorgenommen werden soll. ${ }^{190}$ Dies folgt dem Gedanken, daß sich die Zahlungen dieser drei Phasen besonders stark voneinander unterscheiden, während sie sich innerhalb der jeweiligen Phase sehr ähnlich sind. Im Zeitverlauf findet man hauptsächlich regelmäßige Zahlungen von wiederkehrender Natur, während zu Beginn und Ende der Immobilieninvestition häufig Einmalzahlungen anfallen. ${ }^{191}$

Im Investitionszeitpunkt existieren aus Sicht des Käufers in erster Linie Auszahlungen. Den größten finanziellen Umfang hat der Kaufpreis. Ihm wird an dieser Stelle keine weitere Beachtung geschenkt, weil der Grenzpreis das Ergebnis der Bewertung ist. Das Modell sucht den gerade noch verschmerzbaren Grenzpreis für die Übertragung des Eigentums. ${ }^{192}$

Weil beim Kauf einer Eigentumswohnung auch Erwerbsnebenkosten anfallen, muß sorgfältig zwischen einerseits dem Grenzpreis einschließlich Transaktionskosten und andererseits der Schmerzgrenze für die Preisverhandlung unterschieden werden: Der Grenzpreis ist der Gesamtbetrag, den man maximal bezahlen kann, ohne daß das Geschäft unvorteilhaft wird. ${ }^{193}$ Die Transaktionskosten erhält der Verkäufer nicht. Mit ihm verhandelt man ausschließlich über den reinen Kaufpreis. Um die Konzessionsgrenze für die Verhandlung zu kennen, muß der präsumtive Käufer den Grenzpreis bereinigen. Wegen der prozentualen Abhängigkeit der Erwerbsnebenkosten vom Kaufpreis ist dieser Schritt meist unproblematisch: ${ }^{194}$ Notar- und Grundbuchkosten sind verbindlich im GNotKG geregelt und betragen zusammen etwa 1,5 Prozent. ${ }^{195}$ Die Grunderwerbsteuer beträgt nach GrEStG 3,5 Prozent, wobei die Bundesländer abweichende Regelungen

${ }^{190}$ So auch ROPETER (1998), S. 245 ff., TOLL/WALOCHNIK (2013), S. 22, ROTTKE/KRAUTZ(2017), S. 802.

${ }^{191} \mathrm{Vgl}$. SCHULTE/SOTELO/ALLENDORF/ROPETER-AHLERS/LANG (2016), S. 584 ff.

${ }^{192}$ Dabei ist streng zwischen Wert und Preis zu unterscheiden: Der vom Modell berechnete Grenzpreis verkörpert die äußerste Grenze der Zahlungsbereitschaft und ist allenfalls ein potentieller Preis, vgl. MOXTER (1983), S. 5. Er reflektiert den vom Bewertungsobjekt ausgehenden Nutzen, vgl. MANDL/ RABEL (1997), S. 68, MATSCHKE (2017a), S. 4. Das Modell dient jedoch nur zur Entscheidungsunterstützung, vgl. HAX (1964), S. 430. Welcher Preis im Fall einer Einigung tatsächlich gezahlt wird und ob überhaupt eine Einigung erzielt werden kann, wird mit dem Entscheidungswert nicht beantwortet, sondern hängt u.a. vom Verhandlungsgeschick beider Parteien ab, vgl. KUßMAUL (1996), S. 266.

${ }^{193}$ Der Algorithmus maximiert diese Variable und erhöht ihren Wert so lange, bis die Vorteilhaftigkeit der Transaktion gerade noch nicht kippt. An diesem Punkt besteht Indifferenz zwischen Durchführung und Unterlassung. Weil das Geschäft jenseits dieser Grenze nicht mehr lohnenswert wäre, können zusätzliche Transaktionskosten nicht gezahlt werden. Konsequenterweise muß $p^{*}$ bereits alle Transaktionskosten enthalten.

${ }^{194}$ Auch zum Folgenden vgl. REHKUGLER (2011b), S. 249, SCHULZE/STEIN/TIETGEN/MÖLLER (2017), S. $28 \mathrm{ff}$.

${ }^{195}$ Vgl. HELLERFORTH (2008), S. 1, ROTTKE (2017a), S. 94 f., LINDMAYER/DIETZ (2018), S. 288. 
treffen können: ${ }^{196}$ In Nordrhein-Westfalen beträgt sie derzeit beispielsweise 6,5 Prozent. ${ }^{197}$ War ein Immobilienmakler in die Vermittlung involviert, fällt Maklercourtage an. Ihre Höhe ist nicht gesetzlich geregelt, oft beträgt sie 3,57 Prozent. ${ }^{198}$ Somit kann der gesamte Grenzpreis zur Bereinigung um Transaktionskosten mit einem Faktor zwischen 1,08 und 1,115 umgerechnet werden.

\subsection{Zahlungen während der Investitionsdauer}

\subsection{Verhältnis zum Mieter}

Weil sich jeder vermietende Eigentümer im Spannungsfeld zwischen vertraglichem Mietverhältnis und gesellschaftlichem Verhältnis zur Wohnungseigentümergemeinschaft befindet, sollen die folgenden Ausführungen ebenfalls dahingehend untergliedert werden. Zu den wichtigsten Eingangsdaten im Zeitablauf gehört die Höhe der Mieteinnahmen. ${ }^{199}$ Der Mietzins ist die monetäre Gegenleistung für die Überlassung der Mietsache. ${ }^{200}$ Sie umfaßt Wohnraum und i.d.R. auch weitere Flächen, wie z.B. Kellerräume, Garagen oder PKW-Stellplätze. ${ }^{201}$ In der Regel sind die Mieteinnahmen die einzige Einnahmequelle des Vermieters aus seiner Investition. ${ }^{202}$

Wenn eine Wohnung in bereits vermietetem Zustand erworben wird, vereinfacht es die Bewertungssituation insoweit, als zumindest die Höhe der aktuellen Mieteinnahmen bekannt ist. ${ }^{203}$ Darüber hinaus besteht Klarheit hinsichtlich des bisherigen Zahlungsverhaltens des Mieters. Falls keine Selbstnutzung geplant ist, muß der Investor den bestehenden Mietvertrag übernehmen: „Kauf bricht nicht Miete“, sagt § 566 BGB. ${ }^{204}$

Im Falle einer Neuvermietung wird die Höhe der Mieteinnahmen vor allem von Objektund Standortfaktoren in Zusammenspiel mit der ortsüblichen Miete determiniert. Das

${ }^{196}$ Vgl. VAN SUNTUM/SCHULTEWOLTER (2014), S. 207 f., LINDAUER (2016), S. 101 f., IBEL (2017), S. 131.

${ }^{197}$ Vgl. LINDMAYER/DIETZ (2018), S. 428 f., VOIGTLÄNDER (2019), S. 120. Zur Grunderwerbsteuer vgl. SCHNEELOCH/MEYERING/PATEK (2017b), S. 57 ff., FISCHL (2017c).

${ }^{198}$ Vgl. KELLER (2013a), S. 223. Zum Berufsstand des Maklers, insbesondere im historischen Kontext, vgl. GERBAULET (2017). Im Zeitpunkt des Erwerbs können auch Such-, Recherche- und Besichtigungskosten entstehen, vgl. FELDMANN/GERSTNER/HOFMANN/ISENHÄFER/SEGERER/VÄTH (2016), S. 386 f. Zu diesen gehören Pauschalen für gefahrene Kilometer. Man denke auch an Kosten der Entscheidungswertermittlung, sei es für hinzugezogene Berater oder die Opportunität der eigenen, investierten Zeit. Möglicherweise fallen auch Handwerkerkosten an, um die Immobilie in vermietbaren Zustand zu versetzen, vgl. SCHULZE/STEIN/TIETGEN/MÖLLER (2017), S. 125.

199 Auch zum Folgenden vgl. SCHULTE/SOTELO/ALLENDORF/ROPETER-AHLERS/LANG (2016), S. 586, VON BRESSENSDORF (2016), S. 267, MARKMANN/MARKMANN/ROTTKE (2017), S. 642.

${ }^{200} \mathrm{Vgl}$. HELLERFORTH (2012), S. 8, BLANK (2017a), Rn. 604, ZEHELEIN (2019), Rn. 511.

${ }^{201}$ Vgl. WIEGNER (2019), Rn. 5, SCHMIDT (2019), S. 302 ff.

${ }^{202} \mathrm{Vgl}$. MARKMANN/MARKMANN/ROTTKE (2017), S. 642, ähnlich VON BRESSENSDORF (2016), S. 246.

${ }^{203}$ Vgl. PFLÜGL (2017e), Rn. 12 ff., FESSELMANN/KOBABE/SCHWENZFEIER/SIEGEMUND (2018), S. 97.

${ }^{204}$ Vgl. dazu HÄUBLEIN (2016c), STREYL (2017). 
öffentliche Meinungsbild bzgl. eines Standortes beeinflußt dessen Attraktivität und die dort herrschende Angebots- und Nachfragesituation. ${ }^{205}$ Zusätzlich wird die Nachfrage wesentlich von individuellen Gebäudefaktoren determiniert. Dazu gehören Alter, Bauqualität, Ausstattung, Nutzungsmöglichkeiten sowie aktueller Zustand. ${ }^{206}$

Für die Prognose der zu erwartenden Miete im Falle der Neuvermietung stehen u.a. Mietspiegel zur Verfügung. Diese werden von Gutachterausschüssen erstellt und geben Auskunft über ortsübliche Durchschnittsmietpreise einer Stadt oder Gemeinde. ${ }^{207}$ Daneben gibt es zahlreiche Veröffentlichungen von großen Maklergesellschaften oder Fachzeitschriften. ${ }^{208}$ Nicht zuletzt findet man im Netz multimediale Landkarten zum Abruf durchschnittlicher Mietpreise eines bestimmten Standorts bzw. Stadtteils. Bei Neuvermietung erfolgt die Mieteranalyse und Mieterauswahl oft bonitätsabhängig, ausschlaggebend ist insbesondere die Einkommenssituation. ${ }^{209}$ Allerdings muß vor einer rein quantitativen Sichtweise gewarnt werden, weil sie zur Ausblendung anderer Faktoren verleitet: Oft unterschätzt man den Stellenwert von Miettreue, geringer Fluktuation und einem guten Verhältnis zum Mieter. ${ }^{210}$

Die Möglichkeit von Mieterhöhungen im Laufe der Zeit können ebenfalls berücksichtigt werden. Jedoch sind sie bei bestehenden Mietverträgen über Wohnraum nur in einem engen, gesetzlich reglementierten Rahmen möglich. ${ }^{211}$ Zudem machen private Kleinvermieter nur höchst selten von dieser Möglichkeit Gebrauch. ${ }^{212}$ Man unterscheidet folgende Arten der Mieterhöhung:

- Anpassung an die ortsübliche Vergleichsmiete (normale Mieterhöhung), ${ }^{213}$

- Indexmiete (Wertsicherungsklausel, z.B. Bindung an einen Verbraucherpreisindex), ${ }^{214}$

- Staffelmiete ${ }^{215}$ (eine bereits im Mietvertrag fest vereinbarte Mieterhöhung zu bestimmten Zeitpunkten) und

${ }^{205}$ Vgl. SCHULTE/HOLZMANN (2005), S. 40, KURZROCK (2017b), S. 743 ff., FESSELMANN/KOBABE/ SCHWENZFEIER/SIEGEMUND (2018), S. 6, ERTLE-STRAUB (2019), S. 373.

${ }^{206}$ Vgl. HAAS (2010), S. 46, KELLER (2013b), S. 25 f., SCHARMANSKI/WIENCKE (2017), S. 736 f.

${ }^{207}$ Vgl. BUSCH(2017a), S. 692, VOIGTLÄNDER (2019), S. 148 f.

${ }^{208}$ Einen Überblick geben FELDMANN/GERSTNER/HOFMANN/ISENHÄFER/SEGERER/VÄTH (2016), S. 387, SCHARMANSKI/ WIENCKE (2017), S. $718 \mathrm{ff}$.

${ }^{209}$ Vgl. TRÜBESTEIN/PRUEGEL (2012), S. 38, MARKMANN/MARKMANN/ROTTKE (2017), S. 643 ff.

${ }^{210}$ Vgl. dazu SCHULZE/STEIN/TIETGEN/MÖLLER (2017), S. 126.

${ }^{211}$ Vgl. KREUTER-KIRCHHOF (2014), S. 109 ff., ROTTKE/EIBEL (2017), S. 842, zu den Formalitäten vgl. HÜLSMANN (2018), S. 15, zur Mietpreisbremse vgl. KREUTZER (2015), BÖRSTINGHAUS (2018).

${ }^{212} \mathrm{Vgl}$. SCHULZE/STEIN/TIETGEN/MÖLLER (2017), S. 124.

${ }^{213}$ Vgl. zu dieser Form der Mieterhöhung ARTZ (2016a), BÖRSTINGHAUS (2017a), FLEINDL (2019), SIEGMUND (2019a).

${ }^{214}$ Dazu vgl. VON BRESSENSDORF (2016), S. 270 f., BÖRSTINGHAUS (2017b), Rn. 44 ff.

${ }^{215} \mathrm{Zu}$ dieser gewissermaßen automatischen Form der Mieterhöhung vgl. BUSCH (2017a), S. 692, SIEGMUND (2019b). 
- Mieterhöhung im Rahmen von Modernisierungsmaßnahmen. ${ }^{216}$

Als weitere Determinanten sollten Risikofaktoren berücksichtigt werden, die zum Ausfall der Mieteinnahmen im Zeitverlauf führen können. ${ }^{217}$ Steht die Wohnung leer, fallen nicht nur Mieteinnahmen, sondern auch Vorauszahlungen für Betriebskosten vollständig aus. Kreditraten müssen jedoch weiterhin bedient und das Hausgeld fortlaufend an die WEG bezahlt werden. Leerstand kann seine Ursachen in gewöhnlicher Fluktuation haben. Denkbar sind aber auch anhaltende Probleme, geeignete Mieter zu finden. ${ }^{218}$

Ein (vollständiger oder teilweiser) Mietausfall einer bewohnten Eigentumswohnung kann entweder auf eine berechtigte Mietminderung aufgrund von Mängeln ${ }^{219}$ oder auf die Zahlungsunfähigkeit oder -unwilligkeit des Mieters ${ }^{220}$ zurückzuführen sein. In beiden Fällen ist schnelles Handeln obligatorisch: Zwar ist in den letztgenannten Fällen eine einvernehmliche Lösung wünschenswert, damit der Mieter seinen Pflichten nunmehr nachkommt. Ist jedoch keine Einigung abzusehen, muß er im Rahmen rechtlicher Möglichkeiten schnellstens aus der Wohnung entfernt und durch einen solventen Mieter ersetzt werden, damit größerer finanzieller Schaden vermieden wird. Nicht nur die Ausgaben für Hausgeld und Kreditrate laufen weiter. Schlimmer noch: Eine Neuvermietung ist unmöglich, weil die Wohnung noch bewohnt ist. Zusätzlich kommen Rechtsanwaltsund nach Räumung der Wohnung ggf. auch Reparaturkosten auf den Vermieter zu. Gesetzliche Rahmenbedingungen, um die Rechte des Vermieters gegenüber dem Mieter geltend zu machen, beeinflussen die finanziellen Verlustrisiken wesentlich. Dazu gehören Vermieterpfandrechte ${ }^{221}$, Kündigungs- und Räumungsfristen. ${ }^{22}$ Auch die Höhe verwertbarer Sicherheiten, z.B. einer Mietkaution ${ }^{223}$, ist hier von Bedeutung.

\footnotetext{
${ }^{216}$ Vgl. hierzu Unterkapitel 4.7.

${ }^{217}$ Auch zum Folgenden vgl. LEIFELS/RAFFELHÜSCHEN (2014), S. 144, SCHULTE/SOTELO/ALLENDORF/ ROPETER-AHLERS/LANG (2016), S. 587, LEOPOLDSBERGER/THOMAS/NAUBEREIT (2016), S. 445 f.

${ }^{218}$ Wegen der Nichtsubstituierbarkeit des Gutes Wohnraum ist dieses Problem häufig auf eine schlechte Entwicklung von Standort oder Objekt (z.B. Sanierungsstau) zurückzuführen, vgl. HAAS (2010), S. 46, DRASDO (2017a), Rn. 120, MÜLLER (2019), Rn. 54.
}

${ }^{219}$ Vgl. zur Mietminderung aufgrund von Mängeln HäUBLEIN (2016b), DRASDO (2018b).

${ }^{220}$ Vgl. TRÜBESTEIN/PRUEGEL (2012), S. 38.

${ }^{221}$ Vgl. zum Vermieterpfandrecht ARTZ (2016b), Rn. 5 ff., TEICHMANN (2018), BRUNS (2019), HUBER (2019), S. $232 \mathrm{ff}$.

${ }^{222}$ Zur fristlosen Kündigung vgl. BUSCH(2017a), S. 699, BLANK (2017b).

${ }^{223}$ Zur Mietkaution vgl. THEESFELD (2011), ZEHELEIN (2019), Rn. 181. Zu Rechten bzgl. der Mietkaution nach Beendigung des Mietvertrags vgl. WETEKAMP (2007), Rn. 73 ff., zur Mietkaution bei Eigentümerwechsel vgl. KREUZAU (2011). 
Zwar können vor allem bestehende Mietverhältnisse hinsichtlich der Mieterbonität analysiert werden. ${ }^{224}$ Dies ist jedoch nur eine Seite der Medaille: Objektzustand, Eigentümergemeinschaft und Verwaltung kommt ebenfalls ein hoher Stellenwert zu. ${ }^{225}$ Bei Mietminderung aufgrund von Mängeln hängt die Höhe des finanziellen Schadens von der Möglichkeit ab, den vertragsgemäßen Zustand der Mietsache durch Reparaturen wiederherzustellen, weil vielfältige, teils enge Verflechtungen zwischen Sonder- und Gemeinschaftseigentum bestehen. ${ }^{226}$

Auch bei unproblematischen Mietverhältnissen verursacht jede Eigentumswohnung eine Reihe laufender Kosten. Jedoch kann der Vermieter nur solche Positionen an seinen Mieter weitergeben, die in der Betriebskostenverordnung (BetrKV) genannt sind. ${ }^{227}$ Diese durch laufenden Gebrauch entstehenden Kosten bilden die Obergrenze der auf den Mieter umlagefähigen Nebenkosten. ${ }^{228}$ Zudem muß die Übernahme ausdrücklich im Mietvertrag vereinbart sein. ${ }^{229}$ Für die Mitglieder der Wohnungseigentümergemeinschaft hingegen gibt es eine solche Unterscheidung nicht. Sie müssen unabhängig von der BetrKV oder sonstiger Vorschriften anteilig für sämtliche Kosten aufkommen, die aufgrund der Immobilie anfallen. ${ }^{230}$ Schließlich gibt es keine übergeordnete Instanz wie einen Vermieter, die an ihrer Stelle die Kosten tragen würde.

${ }^{224}$ Vgl. zur Mieteranalyse FELDMANN/GERSTNER/HOFMANN/ISENHÄFER/SEGERER/VÄTH (2016), S. 407, KURZROCK (2017b), S. 748 f., ERTLE-STRAUB (2019), S. 375.

${ }^{225}$ Zur Analyse der WEG vgl. PFLÜGL (2017e), Rn. 20 ff., insbesondere zu den rechtlichen Verhältnissen laut Teilungserklärung und zu ggf. davon abweichender Bauausführung vgl. SCHWERING (2019), S. 291, insbesondere zur Beschlußsammlung vgl. ELZER (2017).

${ }^{226} \mathrm{Vgl}$. Gliederungspunkt 2.2.3.2.2.

${ }^{227}$ Zur Abgrenzung zwischen auf den Mieter umlagefähigen und nicht umlagefähigen Kosten vgl. BLANK (2017e), Rn. 34, DRAGER (2019a), Rn. 17 ff., LEHMANN-RICHTER (2019), S. 105 ff. Einen Überblick zu den Unterschieden zwischen mietrechtlicher Betriebskostenabrechnung und wohnungseigentumsrechtlicher Jahresabrechnung gibt BLANK (2004). Zum Katalog möglicher Betriebskosten vgl. LANGENBERG (2017a), Rn. 102 ff., PFEIFER (2019a), S. 327 ff., DRAGER (2019b), Rn. 5 ff. In Abhängigkeit vom Mietvertrag können meist folgende Betriebskosten auf den Mieter umgelegt werden: Allgemeinstrom, Frisch- und Abwasser, Gebäude- und Haftpflichtversicherungsprämien, Grundsteuer, Kosten von Hausmeister, Gartenpflege und Treppenhausreinigung, Heiz- und Warmwasserkosten (einschließlich Nebenkosten wie Kostenerfassung, Legionellenproben, Schornsteinfeger und Wartung der Heizungsanlage), Müllabfuhr, Niederschlagswasser, gemietete Rauchmelder und gemietete Kaltwasserzähler, Schädlingsbekämpfung, Straßenreinigung, verschiedene Wartungs- und Kontrollkosten (z.B. Dachkontrolle und Rinnenreinigung) und Winterdienst.

${ }^{228}$ Zur BetrKV als Obergrenze der vom Mieter zu tragenden Kosten vgl. PFEIFER (2019a), Rn. 99 f., HUBER (2019), S. $214 \mathrm{f}$.

${ }^{229}$ Andernfalls ist von einer Pauschalmiete einschließlich Betriebskosten auszugehen, vgl. SUILMANN (2017c), Rn. 3, WIEDERHOLD (2019), Rn. 62 f., PFEIFER (2019a), Rn. 63 f.

${ }^{230}$ Diese Ausgaben können vielfältig sein; Beispiele liefern MUSIELACK (2017), S. 74, SUILMANN (2017c), Rn. 8, BECKER (2018c), Rn. 26. Man denke u.a. an Kosten, die im Rahmen von Rechtsstreitigkeiten entstehen, vgl. dazu BÄRMANN/PICK (2010), Rn. 20 ff., SAUREN (2014b), Rn. 12P, oder an Versicherungsschäden, bei denen die Versicherungsgesellschaft zwar die Leistung verweigert, aber dennoch repariert werden muß, vgl. dazu WITTEMANN (2017), S. 352 ff., DÖTSCH (2018a), S. 372, Bußgelder aufgrund von Ordnungswidrigkeiten, z.B. im Rahmen des Meß- und Eichgesetzes, 
Umlagefähige Betriebskosten sind für den vermietenden Eigentümer grundsätzlich ein durchlaufender Posten von untergeordnetem Stellenwert. In der Regel überweist der Mieter monatlich die vertraglich vereinbarte Kaltmiete zzgl. einer monatlichen Vorauszahlung, über die jährlich abgerechnet wird. Auch im Fall der Bewertung eigengenutzter Wohnimmobilien sind Betriebskosten nur dann Gegenstand der Betrachtung, wenn sie sich von Opportunitäten in Form von Nebenkosten vergleichbarer Mietobjekte wesentlich unterscheiden. Bei Leerstand oder Mietausfall sind sie für den Vermieter hingegen von großer Bedeutung, weil er umlagefähige Kosten in diesem Fall zwangsläufig selbst tragen muß. Die Höhe umlagefähiger Nebenkosten ist nur innerhalb gewisser Grenzen beeinflußbar. Sie wird einerseits von der technischen Gebäudeausstattung beeinflu $3 \mathrm{t}^{231}$ und hängt andererseits vom Vorhandensein bestimmter Verträge ab (z.B. Kabelfernsehen oder Hausmeisterdienst). Dennoch können viele Arten von Betriebskosten über das Nutzerverhalten beeinflußt werden. Ein Anreiz zum Energie- und Kostensparen entsteht aber oft nur bei verbrauchsproportionaler Kostenumlage. Die Verbrauchserfassung gelingt jedoch meist nur bei Wasser- und Heizkosten, indem man Heizkostenverteiler, Wasser- oder Wärmemengenzähler einsetzt. ${ }^{232}$ Man denke z.B. an Kosten der Müllabfuhr, die sich in vielen Kommunen aus Grund- und Leerungsgebühr zusammensetzen. Wer Müll vermeidet, spart Geld. Kosten, deren Verbrauchserfassung nicht möglich ist, werden mietrechtlich aber im Verhältnis der Wohnfläche auf die Anwohner umgelegt. So werden Ersparnisse nur unterproportional an die Nutzer weitergegeben. Je kleiner der Anteil der Wohneinheit am Gebäude ist, desto mehr verliert der Anreiz seine Wirkung. Tabelle 5 ordnet Kosten, die nach $\S 2$ BetrKV auf die Mieter umgelegt werden können, nach ihrer Beeinflußbarkeit. ${ }^{233}$

vgl. dazu PFLÜGL (2017d), Rn. 11, WANDERER (2017b), Rn. 97, oder wegen unterlassener Untersuchung des Trinkwassers auf Legionellen, dazu PFLÜGL (2017a), Rn. 43. Ein ungewöhnlicher Fall ereignete sich in Bad Harzburg im Landkreis Goslar, vgl. dazu SÜDDEUTSCHE ZEITUNG (2015): Das Gesundheitsamt ging nicht wie üblich gegen die (sehr große) Wohnungseigentümergemeinschaft vor, sondern schickte rund 300 Anhörungsbögen an die einzelnen Wohnungseigentümer, denen ein Bußgeld von jeweils bis zu 5.000 EUR droht. Hintergrund war die Weigerung der Eigentümerversammlung, von Legionellen befallene Wasserleitungen von einer Fachfirma spülen zu lassen.

${ }^{231}$ Zum Beispiel (teils gesetzlich vorgeschriebene) Wartungen oder TÜV-Prüfungen von Aufzügen, Brandschutztüren, Rauchabzugsanlagen, Tiefgaragentoren, vgl. dazu FRITSCH (2018), Rn. 814 ff.

${ }^{232}$ Auch zum Folgenden vgl. § 556a Abs. 1 BGB, vgl. auch SUILMANN (2017c), Rn. 21, LANGENBERG (2017b), Rn. 20 ff. Weitere gängige Umlageschlüssel sind Personenzahlen oder die Anzahl der Wohneinheiten, vgl. VON HAUFF (2017c), S. 150 f. Im Wohnungseigentumsrecht gilt hingegen grundsätzlich der Miteigentumsanteil als Umlageschlüssel, vgl. BECKER (2018a), Rn. 39 f.

${ }^{233}$ Viele Kosten der zweiten und dritten Kategorie können durch Wechsel des Vertragspartners gesenkt werden, z.B. Gartenpflege, Hausreinigung, Heizkostenerfassung und -abrechnung, Versicherung oder Wartung. Bei aller Kostenersparnis sollte man dennoch stets den goldenen Mittelweg suchen, weil der billigste Anbieter selten der Beste sein dürfte. Überdies kann unterlassene Wartung langfristig erhebliche Folgekosten nach sich ziehen, vgl. MÜLLER (2019), Rn. 54. Dies $m u \beta$ aber keineswegs in allen Fällen so sein, vgl. HURLEBAUS (2013), S. 293. 


\begin{tabular}{|c|c|c|}
\hline $\begin{array}{l}\text { Durch Nutzerverhalten } \\
\text { beeinflußbar }\end{array}$ & $\begin{array}{l}\text { Durch Nutzerverhalten } \\
\text { nicht beeinflußbar }\end{array}$ & $\begin{array}{l}\text { Durch Unterlassung bzw. } \\
\text { Eigenleistung beeinfluß- } \\
\text { bar }\end{array}$ \\
\hline $\begin{array}{ll}\text { - } & \text { Müllabfuhr (Ver- } \\
\text { brauchskosten) } \\
\text { - } \\
\text { Heizung (Verbrauchs- } \\
\text { kosten) } \\
\text { - Warmwasser (Ver- } \\
\text { brauchskosten) } \\
\text { - Frischwasser } \\
\text { - Abwasser } \\
\text { - } \text { Beleuchtung (Strom) } \\
\text { - } \text { Aufzug (Strom) }\end{array}$ & $\begin{array}{ll}\text { - } & \text { Müllabfuhr (Grund- } \\
\text { kosten) } \\
\text { - Heizung (Grundkosten, } \\
\text { Schornsteinfeger, Ver- } \\
\text { brauchserfassung, War- } \\
\text { tung) } \\
\text { - Grundsteuer } \\
\text { - Niederschlagswasser } \\
\text { - Öffentliche Straßenrei- } \\
\text { nigung } \\
\text { - Öffentlicher Winter- } \\
\text { dienst } \\
\text { - Versicherungsprämien } \\
\text { - Aufzug (Wartung und } \\
\text { TÜV) }\end{array}$ & $\begin{array}{l}\text { - Gebäudereinigung } \\
\text { - Hausmeister } \\
\text { - Gartenpflege } \\
\text { - Wartungskosten } \\
\text { - Kabelfernsehen }\end{array}$ \\
\hline
\end{tabular}

Tabelle 5: Umlagefähige Betriebskosten

\subsection{Verhältnis zur Wohnungseigentümergemeinschaft}

Geht man vom klassischen Bild des Vermieters aus, der alleiniger Eigentümer eines Mehrfamilienhauses ist, empfängt er allein sämtliche gebäudebezogenen Rechnungen und bezahlt Nebenkosten wie Wasser, Gas oder Versicherungsprämien zu Lasten seines eigenen Bankkontos. Im Gegenzug erhält er von seinen Mietern Vorauszahlungen für Betriebskosten, über die er einmal im Jahr abrechnet, wobei Ausgaben, die keine Betriebskosten sind, z.B. Reparaturkosten, nicht von den Mietern getragen werden müssen. Auch die Willensbildung ist unkompliziert - egal ob Wärmedämmung der Fassade oder Modernisierung der Heizungsanlage - der klassische Vermieter muß mit niemandem diskutieren oder Auseinandersetzungen über die Kostenverteilung führen. Als Alleineigentümer entscheidet und zahlt er alles selbst.

Dieser Idealtyp ist veraltet, und die Realität sieht inzwischen völlig anders aus: „Mietshäuser werden praktisch keine mehr gebaut. Ihr Bestand ist sogar rückläufig, da sie häufig in Eigentumswohnungen umgewandelt und diese dann an eine Vielzahl von Eigentümern verkauft werden." ${ }^{234}$ Wie schon in Gliederungspunkt 2.2.3.2.2 angesprochen, sind die Dinge bei Wohnungseigentümergemeinschaften komplizierter. Zwar gibt es auch hier sowohl umlagefähige als auch nicht umlagefähige Nebenkosten. Es gibt aber mehr als nur einen Eigentümer. Das Gebäude hat dennoch nur einen Hauptanschluß für Wasser, die Gebäudeversicherung berechnet nur eine grundstücksbezogene Prämie ${ }^{235}$

\footnotetext{
${ }^{234}$ STÜRZER/KOCH/HOPFENSPERGER/STERNS/STERNS-KOLBECK/ZIEGELMAYER (2016), S. 295 f.

${ }^{235}$ Vgl. DÖTSCH (2018a), S. 366.
} 
und die Stadtverwaltung erhebt eine Gebühr für Niederschlagswasser ${ }^{236}$. Zahlungspflichtiger ist stets die Wohnungseigentümergemeinschaft. ${ }^{237}$ Der Verwalter als ihr gesetzlicher Vertreter empfängt sämtliche Rechnungen und begleicht sie zu Lasten eines auf den Namen der WEG geführten Bankkontos. Da er als Treuhänder von fremdem Vermögen fungiert, muß er den Eigentümern nach Jahresende Rechenschaft über dessen Verwendung ablegen. ${ }^{238}$ Dazu dient das Rechenwerk jeder Wohnungseigentümergemeinschaft, bestehend aus Wirtschaftsplan und Jahresabrechnung, ggf. ergänzt um Sonderumlagen. ${ }^{239}$

Um laufende Kosten bezahlen zu können, werden Finanzierungsvorschüsse erhoben, die von den Eigentümern an die WEG zu zahlen sind. ${ }^{240}$ Zur Berechnung von deren Höhe erstellt der Verwalter eine Prognoserechnung, genannt Wirtschaftsplan. ${ }^{241}$ Darunter versteht man eine nach Kostenarten gegliederte Übersicht der erwarteten Auszahlungen für ein Kalenderjahr. Sie dient der Berechnung der monatlichen Vorauszahlungen, die man Wohn- oder Hausgeld nennt. Das Hausgeld geht über die von den Mietern zu tragenden Betriebskostenvorauszahlungen hinaus. ${ }^{242}$ Die Eigentümer müssen für sämtliche Kosten aufkommen, die von Grundstück und Gebäude verursacht werden, seien es Bankgebühren, Kosten der Verwaltung oder von Rechtsstreitigkeiten. ${ }^{243}$

Insbesondere Reparaturkosten können nicht auf den Mieter abgewälzt werden. ${ }^{244} \mathrm{Im} \mathrm{Ge}-$ gensatz zu vielen umlagefähigen Kosten verlaufen diese weit weniger linear und sind

${ }^{236}$ Für diese haften die Wohnungseigentümer sogar gesamtschuldnerisch, vgl. BECKER (2014), S. 15.

${ }^{237}$ Auch zu Folgendem vgl. STÜRZER/KOCH/HOPFENSPERGER/STERNS/STERNS-KOLBECK/ZIEGELMAYER (2016), S. 241 f., DRASDO (2017a), Rn. 73, BECKER (2018a), Rn. 21 ff., GREINER (2019), Rn. 35 f.

${ }^{238}$ Vgl. SAUREN (2014e), Rn. 2.

${ }^{239}$ Vgl. VON HAUFF (2017c), S. 125, HERMANN (2019b), Rn. 35 ff.

${ }^{240}$ Die Zahlungspflicht entsteht, wenn der Verwalter von den Eigentümern auf Grundlage des Wirtschaftsplans Zahlungen anfordert, vgl. WANDERER (2017a), Rn. $51 \mathrm{ff}$. GREINER hält die gesetzliche „,Regelung zur Anforderung von Geld“ nach § 27 Abs. 1 WoEigG für „,missglückt“”, ,unvollständig“ und ,überflüssig“, weil sie in Formalismen versinkt und den Blick für die realen Verhältnisse verliert, vgl. GREINER (2019), Rn. 38. Er empfiehlt, gleichzeitig mit dem Wirtschaftsplan auch die monatliche Fälligkeit der Hausgeldbeiträge zu beschließen, um die Problematik zu umgehen und eine gesonderte Anforderung durch den Verwalter zu erübrigen, vgl. ebd. Eigentümer, die mit ihren Zahlungen im Rückstand sind, gerieten auf diese Weise automatisch in Verzug, vgl. dazu auch WANDERER (2017a), Rn. $51 \mathrm{ff}$.

${ }^{241}$ Vgl. zum Wirtschaftsplan ENGELHARDT (2017d), WANDERER (2017a), BARTHOLOME (2019b).

${ }^{242}$ Das Hausgeld umfaßt neben den Betriebskosten u.a. Verwaltungskosten und Kosten der Instandhaltung und Instandsetzung, vgl. DRAGER (2019a), Rn. 17 ff., sowie die Beitragspflicht zur Instandhaltungsrücklage, vgl. MÜLLER (2015e), Rn. 256. Dazu merkt SUILMANN an: „Die Unterscheide zwischen der wohnungseigentumsrechtlichen Jahresabrechnung und der mietrechtlichen Betriebskostenabrechnung sind Wohnungseigentümern und Mietern vielfach unbekannt, weshalb es immer noch vorkommt, dass Wohnungseigentümer die für sie erstellten Jahreseinzelabrechnungen unverändert als Betriebskostenabrechnungen an ihre Mieter weiterleiten.“ SUILMANN (2017c), Rn. 4.

${ }^{243}$ Vgl. BLANK (2004), S. 371.

${ }^{244}$ Für Reparaturen findet man in der Literatur sehr uneinheitliche Begriffe, die zum Teil nicht abgegrenzt oder definiert, sondern als bekannt vorausgesetzt werden, kritisiert HOMANN (2017), S. 297. 
kaum vorhersagbar. ${ }^{245}$ Sie werden zum Teil von zufällig, kurzfristig und aperiodisch auftauchenden Faktoren determiniert und hängen von Bauqualität, Zustand und Ausstattung des Gebäudes ab - sowie von Maßnahmen, die in der Vergangenheit durchgeführt wurden. Oft wird der Totalausfall der Heizungsanlage als Beispiel für eine plötzlich anstehende Instandsetzungsmaßnahme angeführt. ${ }^{246}$ Folgt man dem Beispiel, würde es den wenigsten Eigentümern gelingen, praktisch über Nacht ihren Anteil des Kaufpreises einer neuen Heizungsanlage aufzubringen, der sich durchaus auf mehrere Tausend Euro summieren kann. Der Verwaltung wäre es nicht ohne weiteres möglich, kurzfristig die erforderlichen Mittel beizutreiben, um den Einbau der neuen Heizung zu beauftragen von Formalitäten und zu fassenden Beschlüssen ganz abgesehen. ${ }^{247}$ Gleichwohl wären

In der wohnungseigentumsrechtlichen Literatur verwendet man häufig das Begriffspaar Instandhaltung und Instandsetzung. Hier besteht überwiegend Konsens, daß Instandhaltung vorbeugende Maßnahmen bezeichnet, während Instandsetzung für die Wiederherstellung des ordnungsgemäßen Zustands steht, vgl. ELZER (2008), S. 153, STÜRZER/KOCH/HOPFENSPERGER/STERNS/STERNS-KOLBECK/ ZIEGELMAYER (2016), S. 66, DRAGER (2019a), Rn. 19 ff. „Oder kürzer: Instandhaltung ist die Vermeidung der Instandsetzung. “ SCHMIDT (2017), Rn. 30. Stellenweise werden aber auch Wartungskosten mit dem Begriff der Instandhaltung vermengt, bspw. bei BECKER (2018a), Rn. 31 ff., HUBER (2019), S. 223. Dies ist sogar naheliegend, schließlich steht Wartung für regelmäßige Inspektionen und Prüfungen der Betriebsbereitschaft, vgl. EHRENHEIM (2017), S. 531, BLANK (2017e), Rn. 34. Eben solche Vorgänge, welche der Pflege und Aufrechterhaltung des ordnungsmäßigen Zustands dienen, bevor Schäden entstehen können. Im mietrechtlichen Sinne ist dieser Unterschied jedoch ausgesprochen wichtig, weil Wartungskosten - anders als Reparaturen - an den Mieter weitergegeben werden können, vgl. PFEIFER (2019a), Rn. 659 ff., SCHMID/ZEHELEIN (2016), Rn. 79. Der mietrechtliche Unterschied zwischen Wartungskosten einerseits und Instandhaltungs- bzw. Instandsetzungskosten andererseits liegt nun darin, daß nur regelmäßig durchzuführende Maßnahmen als Wartungskosten auf den Mieter umgelegt werden können, bspw. die Dachrinnenreinigung oder die Jahresinspektion der Heizung einschließlich Zerlegen und Reinigen des Heizkessels und Prüfung der Abgaswerte, vgl. BLANK (2017e), Rn. 34, SCHMID/ZEHELEIN (2016), Rn. 79. Hingegen sind unter Instandhaltung und Instandsetzung einmalig erforderliche Maßnahmen zu verstehen, wenn ,aufgrund eines Mangels eine Beeinträchtigung der Substanz der Mietsache droht oder eingetreten ist.“ ebd. Maßnahmen zur Erhaltung des ordnungsgemäßen Zustands der Mietsache muß der Vermieter tragen, vgl. BÖRSTINGHAUS (2017d), Rn. 47 f., FLINTROP (2019), Rn. 208, während er bei bestimmten, gesetzlich definierten Modernisierungsmaßnahmen die Möglichkeit zur dauerhaften Mieterhöhung aufgrund von Zustandsverbesserung besitzt, vgl. dazu Abschnitt 4.7.1. In der steuerrechtlichen Literatur wiederum differenziert man zwischen sofort abzugsfähigem Erhaltungsaufwand und nachträglichen Anschaffungs- oder Herstellungskosten, die abgeschrieben werden müssen, vgl. WREDE (2017), S. 79 ff., SIEGERT (2019), S. 328. Beliebtes Thema sind größere Erhaltungsaufwendungen, bei denen ein Wahlrecht zur Abschreibung besteht, vgl. dazu Abschnitt 4.6.1. Aus Gründen der Verständlichkeit verbietet es sich, ein ganzes Bündel von Begriffen ins Feld zu führen, um das gleiche zu beschreiben, daher soll hier nicht das sperrige Begriffspaar Instandhaltung und Instandsetzung verwendet werden. Maßnahmen, die vom Vermieter bzw. der WEG zu tragen sind, sollen grundsätzlich als Instandhaltung bezeichnet werden - und nur eindeutige Wiederherstellungsmaßnahmen bei bereits eingetretenen Schäden als Instandsetzung, während umlagefähige Kosten als Wartung bezeichnet werden sollen.

${ }^{245}$ Vgl. auch zum Folgenden HOMANN (2017), S. 305 f., KURZROCK (2017a), S. 428, SCHARMANSKI/ WIENCKE (2017), S. 736, FESSELMANN/KOBABE/SCHWENZFEIER/SIEGEMUND (2018), S. 7.

${ }^{246}$ Vgl. RENNERT (2012), S. 85, JENNIßEN (2014), S. 199, STÜRZER/KOCH/HOPFENSPERGER/STERNS/ STERNS-KOLBECK/ZIEGELMAYER (2016), S. 79 f., MUSIELACK (2017), S. 70 f., SUILMANN (2017b), Rn. $6 \mathrm{f}$.

${ }^{247} \mathrm{Zu}$ den Formalitäten, die der Verwalter bei Instandhaltung des Gemeinschaftseigentums einhalten muß, gehört insbesondere Information der Eigentümer, Einholung von Kostenvoranschlägen sowie 
vermietende Sondereigentümer ggf. mit Mietminderungen oder gar Hotelkosten größeren Ausmaßes konfrontiert. ${ }^{248}$ Deswegen besteht in den meisten Wohnungseigentümergemeinschaften eine Beitragspflicht zur Instandhaltungsrücklage, deren Beitrag im Hausgeld enthalten ist. ${ }^{249}$ Sie dient dem präventiven Ansparen von Geldmitteln für Instandhaltungsmaßnahmen am Gemeinschaftseigentum. Auch größere Maßnahmen, deren finanzieller Umfang einzelne Eigentümer überfordern könnte, sollen im Notfall schnell finanziert werden können, ohne die WEG in Liquiditätsprobleme zu bringen. ${ }^{250}$

Einberufung einer Eigentümerversammlung, damit die Eigentümer einen Mehrheitsbeschluß über die Reparatur fassen können, vgl. BUB (2009), S. 245, GREINER (2019), Rn. 26 ff.

${ }^{248} \mathrm{Vgl}$. STÜRZER/KOCH/HOPFENSPERGER/STERNS/STERNS-KOLBECK/ZIEGELMAYER (2016), S. 77 und S. 305 f., MERLE (2018a), Rn. 131 f., SUILMANN (2019), S. 115.

${ }^{249}$ Vgl. HOMANN (2017), S. 325 f., DÖTSCH (2018b), S. 67, FRITSCH (2018), Rn. 182 ff. Welche Höhe der Beitragspflicht zur Instandhaltungsrücklage angemessen ist, richtet sich nach dem Einzelfall, z.B. nach Alter und Zustand des Gebäudes oder nach Reparaturanfälligkeit technischer Anlagen, vgl. ENGELHARDT (2017a), Rn. 52, HÜGEL (2019b), Rn. 12 f. Die Literatur verwendet auch hier - häufig abwechselnd - die synonymen Begriffe Instandhaltungsrücklage und Instandhaltungsrückstellung. Zwar ist eine Unterscheidung der Begriffe bei Wohnungseigentümergemeinschaften ,ohne Belang“, sagt MERLE (2018a), Rn. 145. Schließlich stammen die Bezeichnungen aus dem externen Rechnungswesen und sind für reine Kassenrechnungen wie die WEG-Jahresabrechnung, die auf Einnahmen und Ausgaben basieren, gar nicht vorgesehen, vgl. DÖTSCH (2018b), S. 61. Zudem sind Wohnungseigentümergemeinschaften weder Kapitalgesellschaften noch bilanzierungspflichtig, vgl. SUILMANN (2017c), Rn. 27 ff., BECKER (2018c), Rn. 115. Dennoch findet man in der Literatur umfangreiche Diskussionen, welcher Begriff richtig sei. In der betriebswirtschaftlichen Bilanztheorie versteht man unter Rückstellungen zukünftige Verbindlichkeiten, deren Bestehen, Fälligkeit oder Höhe ungewiß sind, vgl. JANSSEN (2009), S. 29, BECKER/PEPPMEIER (2018), S. 247, BAETGE/KIRSCH/THIELE (2019), S. 413. Eine Rücklage hingegen bezieht sich auf eine Position des Eigenkapitals. Aufgrund gesetzlicher Vorschriften müssen Kapitalgesellschaften einen Teil des Jahresüberschusses der gesetzlichen Rücklage zuzuführen, vgl. ERMSCHEL/MÖBIUS/WENGERT (2016), S. 130 f., BECKER/PEPPMEIER (2018), S. 143, BAETGE/KIRSCH/THIELE (2019), S. 100. Insofern ist der Begriff Rücklage zumindest in gewisser Weise für die Ersparnisse einer Wohnungseigentümergemeinschaft passend, vgl. $\operatorname{JENNI\beta EN}(2014)$, S. 199, zumal die Bildung von Rücklagen im bilanziellen Sinne erfolgsneutral ist, vgl. KUßMAUL (2016), S. 390. Zwar verweist HOMANN darauf, daß Wohnungseigentümergemeinschaften keine Gewinnerzielungsabsicht verfolgen und auch der Gesetzgeber in $\S 21$ Abs. 5 WoEigG den Begriff Instandhaltungsrückstellung verwendet, vgl. HOMANN (2017), S. 299 f. Dennoch wirkt es befremdlich, für Verbandsvermögen einen Begriff des Fremdkapitals zu verwenden. Deswegen soll hier der Begriff einer (Instandhaltungs-) Rücklage verwendet werden.

250 „Die zum [Gemeinschaftseigentum] gehörenden Gebäudeteile sollen nicht deswegen verwahrlosen, weil es den [Wohnungseigentümern] an Mitteln fehlt, um umfangreichere Instandhaltungs- oder Instandsetzungsmaßnahmen durchführen zu können, wenn diese nötig werden.“ MERLE (2018a), Rn. 144. Außerdem ist die Rücklage zweckgebunden und für (größere) Instandhaltungsmaßnahmen vorgesehen - und nicht zum Überbrücken von Liquiditätsengpässen, vgl. ENGELHARDT (2017a), Rn. 50, FÖRST (2018), S. 307. Andererseits besteht keine Pflicht, Maßnahmen primär zu Lasten der Rücklage zu finanzieren, auch nicht bei Vorhandensein größerer Ersparnisse. So kann es passieren, daß eine Sonderumlage erhoben wird, wenn von der Mehrheit der Eigentümer nicht gewünscht ist, die vorhandene Instandhaltungsrücklage anzurühren, vgl. STÜRZER/KOCH/HOPFENSPERGER/STERNS/ STERNS-KOLBECK/ZIEGELMAYER (2016), S. 80, FÖRST (2018), S. 304, MERLE (2018a), Rn. 156, $\operatorname{HERMANN}$ (2019b), Rn. 65. 
Wenn die geplanten Vorauszahlungen nicht zur Deckung der Ausgaben ausreichen, können kurzfristige Sonderzahlungen der Eigentümer erforderlich werden. ${ }^{251}$ Man nennt sie Sonderumlage oder Nachtragshaushalt. Sie werden oft bei Liquiditätsschwierigkeiten der WEG erhoben, bspw. bei Zahlungsausfall eines einzelnen Eigentümers oder bei akuten und unvorhersehbaren Instandhaltungsmaßnahmen und leerer Instandhaltungsrücklage. ${ }^{252}$ Aus Geldnot, Unwissenheit oder mangels Daten unterbleibt in Wohnungseigentümergemeinschaften häufig eine langfristige Instandhaltungsplanung. ${ }^{253}$ „Dies wiederum hat zur Konsequenz, dass in der immobilienwirtschaftlichen Praxis Instandhaltungsmaßnahmen häufig als Ad-hoc-Aktionen vorgenommen werden. "254 Zudem gibt es keine gesetzliche Vorschrift hinsichtlich der Höhe der Zuführung zur Instandhaltungsrücklage, weil Instandhaltungsbedarf zu stark von gebäudeindividuellen Faktoren abhängt. ${ }^{255}$ Das Zusammenspiel aus fehlender Planung, einer knappen Rücklage und unerwartet auftretendem Instandhaltungsbedarf kann zu finanziellen Engpässen führen und Sonderumlagen erforderlich machen.

Neben den inhaltlichen gibt es - verglichen mit der mietrechtlichen Betriebskostenabrechnung - auch eine Reihe formeller Besonderheiten der wohnungseigentumsrechtlichen Jahresabrechnung. Nach einem Urteil des Bundesgerichtshofs ${ }^{256}$ ist eine deutliche Abgrenzung zwischen Wirtschaftsplan und Jahresabrechnung erforderlich, die manche Wohnungseigentümer verständlicherweise irritiert. ${ }^{257}$ Meistens möchte ein Wohnungseigentümer den Betrag erfahren, der mit ihm verrechnet wird. Dieser sogenannte Saldo

${ }^{251}$ Auch zu Folgendem vgl. STÜRZER/KOCH/HOPFENSPERGER/STERNS/STERNS-KOLBECK/ZIEGELMAYER (2016), S. 77, WANDERER (2017a), Rn. 78, BARTHOLOME (2019b), Rn. 27.

${ }^{252}$ Vgl. MUSIELACK (2017), S. 82, ähnlich BECKER (2018c), Rn. 44.

${ }^{253}$ Vgl. BRÜBACH (2005), S. 75, MARKMANN/MARKMANN/ROTTKE (2017), S. 648.

${ }^{254}$ HOMANN (2017), S. 303. „In der Praxis wird aus Liquiditäts- und Renditegesichtspunkten häufig auf Instandhaltungs- und Modernisierungspläne [...] und die Bildung von Rücklagen verzichtet. Statt dessen werden im Rahmen der Instandhaltung nur augenscheinliche oder akut notwendige Reparaturen ausgeführt. Eine solche kurzfristig umsetzbare, rein reaktive Instandhaltungsstrategie führt tendenziell langfristig zu Substanzverschlechterungen und daraus resultierender Verschlechterung der Wettbewerbsposition zur Mieterakquise.“ MARKMANN/MARKMANN/ROTTKE (2017), S. 648.

${ }^{255}$ Vgl. ENGELHARDT (2017a), Rn. 52, MERLE (2018a), Rn. 144.

${ }^{256}$ Vgl. BGH, Urteil vom 01.06.2012, V ZR 171/11.

${ }^{257}$ Vgl. zu Folgendem CASSER/SCHULTHEIS (2017a), S. 9, SUILMANN (2017c), Rn. 6, HERMANN (2019b), Rn. 35 ff. Zur Jahresabrechnung vgl. WANDERER (2017b), BECKER (2018c), Rn. 99 ff. Es handelt sich um eine reine Kassenrechnung über die Geldbewegungen auf dem WEG-Konto, vgl. VON HAUFF (2017c), S. 125 ff., SUILMANN (2017c), Rn. 22. Unbedeutend ist die Zugehörigkeit der Kosten zur Abrechnungsperiode. Die einzige - wenn auch sehr bedeutsame - Ausnahme bilden Heizkosten. Sie müssen laut $\S 6$ HeizKV nach dem Leistungsprinzip abgerechnet werden. Für Kosten von Heizung und Warmwasser dürfen ausschließlich die Kosten der im Abrechnungszeitraum verbrauchten Brennstoffe angesetzt werden, vgl. NOACK/WESTNER (2015), S. 96 f., STÜRZER/KOCH/HOPFENSPERGER/STERNS/STERNS-KOLBECK/ZIEGELMAYER (2016), S. 87, VON HAUFF (2017c), S. 129 f. Die Regelungen der HeizKV sind sowohl im Mietrecht als auch im Wohnungseigentumsrecht zwingend anzuwenden, vgl. NOACK/WESTNER (2015), S. 23, PFLÜGL (2017b), Rn. 4 f. Sie genießen Vorrang vor allen anderen vertraglichen Vereinbarungen, also auch vor der Teilungserklärung, vgl. HUBER (2019), S. $220 \mathrm{f}$. 
wird ihm immerhin nachrichtlich mitgeteilt. ${ }^{258}$ Es handelt sich um den Unterschied zwischen tatsächlich angefallenen Kosten und tatsächlich geleisteten Vorauszahlungen. Beschlußgegenstand der Jahresabrechnung ist jedoch die Abrechnungsspitze, welche die Differenz zwischen den (geschuldeten) Soll-Vorauszahlungen und den (tatsächlich angefallenen) Ist-Kosten beschreibt. ${ }^{259}$ Ein potentieller Rückstand gegenüber dem Wirtschaftsplan, d.h. der Unterschied zwischen (geforderten) Soll- und (geleisteten) Ist-Vorauszahlungen ist kein Bestandteil der Jahresabrechnung. Der Wirtschaftsplan wurde bereits auf der vorangegangenen Eigentümerversammlung beschlossen und begründet eine eigenständige, juristisch durchsetzbare Anspruchsgrundlage für die Vorauszahlungen. Er hat eine eigene Verjährungsfrist, auf deren Fundament der Verwalter Zahlungsrückstände - bereits während des Jahres - beitreiben muß. ${ }^{260}$ Eine bereits bestehende Forderung, namentlich ein Zahlungsrückstand, kann im Rahmen der Jahresabrechnung nicht erneut beschlossen und damit auch nicht verrechnet werden. ${ }^{261}$ Ein anderslautender Beschluß wäre ,,insoweit (teil)nichtig, da ansonsten unter Umgehung der Verjährungsregelungen des BGB eine Duplizierung von Forderungen mit Blick auf das Fortbestehen der (unbeglichenen) bereits beschlossenen Beitragsverpflichtungen erfolgt ${ }^{\star{ }^{*} 262}$.

Ein Beispiel, das von folgenden Annahmen ausgeht, mag dies verdeutlichen: Der bestehende Wirtschaftsplan verpflichtete den Wohnungseigentümer bereits während des abgelaufenen Kalenderjahres zur Zahlung von insgesamt 3.000 EUR Hausgeld (Soll-Vorauszahlung). Da er vergessen hatte, den Dauerauftrag zu ändern, und nur 2.700 EUR bezahlt hat (Ist-Vorauszahlung), wäre es Aufgabe des Verwalters gewesen, den Rückstand von 300 EUR rechtzeitig einzufordern (Rückstand zum Wirtschaftsplan):

${ }^{258} \mathrm{Vgl}$. FRITSCH (2018), Rn. $204 \mathrm{ff}$.

${ }^{259}$ Vgl. auch zum Folgenden CASSER/SCHULTHEIS (2017b), S. 11, BECKER (2018c), Rn. 57 ff. und Rn. 101.

${ }^{260}$ Hieraus erwächst auch das Problemfeld der Jahresabrechnung bei Eigentümerwechsel, vgl. dazu VON HAUFF (2017c), S. 152 ff., WANDERER (2017b), Rn. 146 ff., FRITSCH(2018), Rn. 167 ff. Ein Beschluß der WEG entfaltet nur gegenüber denjenigen Eigentümern Bindungswirkung, die zum Zeitpunkt der Beschlußfassung im Grundbuch eingetragen sind. Dies widerspricht der gewöhnlichen Vorstellung einer Stichtagsabrechnung, zur anteiligen Aufteilung der Kosten auf Käufer und Verkäufer. „Eine solche Aufteilung von Kosten, wie sie bei der Abrechnung von Betriebskosten im Mietverhältnis zwingend ist, ist dagegen wohnungseigentumsrechtlich unzulässig“, erklärt SUILMANN (2017c), Rn. 50, und führt weiter aus: „Schon gar nicht darf [der Verwalter] die im Wirtschaftsjahr angefallen Kosten anteilig auf den Veräußerer und den Erwerber verteilen." Dies betrifft jedoch das Verhältnis zwischen neuem Eigentümer und Wohnungseigentümergemeinschaft. Meist ist im notariellen Kaufvertrag geregelt, wie der Betrag im Verhältnis zwischen Käufer und Verkäufer aufzuteilen und auszugleichen ist. VON HAUFF unterbreitet den sinnvollen Vorschlag, drei Abrechnungen zu erstellen, nämlich die echte, WEG-rechtliche Einzelabrechnung sowie zwei an den Eigentumsübergang angepaßte Zwischenabrechnungen. Letztgenannte besitzen zwar keine rechtliche Relevanz und müssen auch entsprechend gekennzeichnet werden. Dennoch unterscheiden solche Zusatzleistungen professionelle Verwalter von allen übrigen, vgl. VON HAUFF (2017c), S. 153.

${ }^{261}$ Vgl. CASSER/SCHULTHEIS (2017a), S. 9, FRITSCH (2018), Rn. 166, HERMANN (2019b), Rn. 154.

${ }^{262}$ FRITSCH (2018), Rn. 204. 
Ist-Vorauszahlung

2.700 EUR
./. Soll-Vorauszahlung = Rückstand

$-3.000 \mathrm{EUR}=-300 \mathrm{EUR}$

Nach Ablauf des Kalenderjahres ergeben sich tatsächliche, auf die Wohneinheit entfallende Kosten von 2.600 EUR (Ist-Kosten). Die Jahresabrechnung stellt diese den geschuldeten 3.000 EUR gegenüber (Soll-Vorauszahlung). Die Differenz beträgt 400 EUR zugunsten des Eigentümers (Abrechnungsspitze).

$\begin{array}{lll}\text { Soll-Vorauszahlung } & \text {./. Ist-Kosten } & =\text { Abrechnungsspitze } \\ 3.000 \text { EUR } & -2.600 \text { EUR } & =+400 \text { EUR }\end{array}$

Das vorgenannte BGH-Urteil räumt dem Eigentümer einen Anspruch gegenüber der WEG auf Erstattung der vollen 400 EUR ein, die mit dem Beschluß fällig geworden sind. Hiervon unabhängig bleibt der gegen ihn lautende Anspruch aufgrund rückständiger Zahlungen in Höhe von 300 EUR bestehen. Es obliegt jetzt dem Verwalter, rechtzeitig die gegenseitige Aufrechnung beider Forderungen zu erklären, weil sie verschiedenen Verjährungen unterliegen. ${ }^{263}$ Für den Eigentümer ergibt sich ein Guthaben in Höhe von 100 EUR (Saldo).

$$
\begin{array}{lll}
\text { Ist-Vorauszahlung } & \text {./. Ist-Kosten } & =\text { Saldo } \\
2.700 \mathrm{EUR} & -2.600 \mathrm{EUR} & =+100 \mathrm{EUR}
\end{array}
$$

Schon bei der Vorselektion potentieller Investitionsobjekte muß der Investor ein tieferes Verständnis des dreigliedrigen WEG-Rechenwerks aufbringen, das aus Jahresabrechnung, Wirtschaftsplan und ggfs. Sonderumlagen besteht. ${ }^{264}$ Bei sorgfältiger Analyse kann er bereits im Entscheidungszeitpunkt Objekte aussortieren, deren finanzielle Lage instabil erscheint. Hinweise darauf findet er in den Abrechnungsunterlagen u.a. in Form von größeren Zahlungsrückständen oder leeren Instandhaltungsrücklagen. Zudem ist erkennbar, ob der Verwalter sein Handwerk beherrscht und rechtliche Vorgaben bei Erstellung der Jahresabrechnung umsetzt. Davon ist nicht zwingend auszugehen. ${ }^{265}$ Es

${ }^{263}$ Weil der Wirtschaftsplan üblicherweise ein Jahr vor der Jahresabrechnung beschlossen wurde, endet sein Fristlauf entsprechend früher, obwohl beide einer dreijährigen Verjährungsfrist unterliegen, vgl. BECKER (2018c), Rn. 97 f.

${ }^{264} \mathrm{Vgl}$. HERMANN (2019b), Rn. $35 \mathrm{ff}$.

${ }^{265}$ Der DACHVERBAND DEUTSCHER IMMOBILIENVERWALTER E.V. führte 2017 eine Umfrage unter 400 Immobilienverwaltern zu fehlerhafter Vorverwaltung durch. Aus den Ergebnissen schlußfolgerte man einen jährlichen Schaden durch unprofessionelle und fehlerhafte Immobilienverwaltung auf 25 Mio. EUR und weitere 55 Mio. EUR für die Aufarbeitung mangelhafter Unterlagen durch die Nachfolgeverwaltung. Damit nicht genug: „Knapp 34 Prozent der [Verwaltungs-] Unternehmen lehnen WEGs aber auch wegen unqualifizierter Vorverwaltungen ab. Wenn es dennoch zur Übernahme einer solchen WEG kam, stellten die Unternehmen fest, dass die häufigsten Fehlerquellen eine unkorrekte Buchführung (76 Prozent), ein Instandhaltungs- und Sanierungsstau (75 Prozent), nicht umgesetzte Beschlüsse der Eigentümer (68 Prozent) und die Nichteinhaltung von gesetzlichen Auflagen (57 Prozent) waren. Auch wurde[n] oft eine fehlerhafte Beschlussfassung (52 Prozent), fehlende Abrechnungen (48 Prozent) und Mängel bei der Beschlusssammlung (35 Prozent) identifiziert.“ DACHVERBAND DEUTSCHER IMMOBILIENVERWALTER E.V. (2017), S. 37. 
kann ggf. ratsam sein, vergangene Wirtschaftspläne mit den korrespondierenden Jahresabrechnungen zu vergleichen, um Rückschlüsse auf Prognosequalität oder unerwartete Einflüsse zu ziehen.

Die Prognose der für ein Modell benötigten zukünftigen Zahlungsströme sollte der Investor jedoch nicht auf Basis dieses mehrschichtigen Rechenwerks vornehmen, weil er Vorauszahlungen, Rückstände und Verrechnungsbeiträge in Beziehung setzen müßte. In der langfristigen Betrachtung sind deren Unterschiede ohnehin nachrangig, weil zwischen Eigentümer und WEG ein jährlicher Ausgleich von Kosten und Vorauszahlungen erfolgt, ebenso zwischen Vermieter und Mieter. In das Bewertungsmodell sollen nur solche Beträge eingehen, die beim Investor tatsächlich zu- oder abfließen. Für eine gezieltere Datensuche empfiehlt sich eine Unterteilung zwischen umlagefähigen und nicht umlagefähigen Kosten, ergänzt um die in letzteren enthaltenen Instandhaltungskosten.

Besonderes unproblematisch erscheinen umlagefähige Kosten, die er zwar mit dem Hausgeld an die WEG bezahlt, seinerseits aber an den Mieter weiterreichen kann. ${ }^{266} \mathrm{Sie}$ sind lediglich im Fall eines Leerstands von Bedeutung. Meist schwanken diese im Zeitablauf nur geringfügig. Anhaltspunkte für die Prognose bieten Mittelwerte aus vergangenen Jahresabrechnungen, sofern der Verkäufer sie zur Verfügung stellt. Andernfalls kann der Rückgriff auf quadratmeterbezogene Erfahrungs- oder Durchschnittswerte vertretbar sein, wenn der Aufwand einer anderweitigen Recherche in keinem Verhältnis zu ihrem Nutzen steht. ${ }^{267}$ Hingegen wirken sich die nicht auf den Mieter umlagefähigen Kosten auf den erzielbaren Zukunftserfolg aus. Abgesehen von Instandhaltungskosten ist die genannte Vorgehensweise auch bei diesen Kosten sinnvoll. Zu ihnen gehören z.B. Verwaltungs- und Bankgebühren, die selten größeren Schwankungen unterliegen.

Jedoch dürfte die Prognose künftiger Instandhaltungsmaßnahmen heillos ausufern. ${ }^{268} \mathrm{Zu}$ stark ist der Einfluß von kleinteiligen, gebäudeindividuellen Faktoren. Aufgrund des finanziellen Umfangs solcher Maßnahmen ist es theoretisch zwar sehr wichtig, deren Kosten und Zeitpunkt abzuschätzen. Immobilien beherbergen aber eine Vielzahl unterschiedlichster Gewerke. Um abzuschätzen, wie haltbar z.B. Heizung, Dach oder Abwasserleitungen sind, wann sie möglicherweise saniert werden müssen oder welche Kosten dies hervorriefe, wären zeit- und kostenintensive Besichtigungen jedes Gewerks durch verschiedene fachspezifische Sachverständige erforderlich. Nur so könnte man Verarbeitungsqualität, Haltbarkeit und Restnutzungsdauer auch nur ansatzweise einschätzen. Deren Hinzuziehung ist kostenintensiv, aber keineswegs ausreichend: Wichtig ist die Einschätzung, ob die anderen Eigentümer bei Instandhaltungsbedarf schnell handeln oder ob sie selbst notwendige Reparaturen immer wieder aufschieben. Das Bewertungssubjekt kann zwar Jahresabrechnungen und Protokolle vergangener Eigentümerversammlungen sichten. Auch Gespräche mit Eigentümern und Verwaltung können wichtige Hinweise geben. Wurden in der Vergangenheit notwendige Instandhaltungen aus

\footnotetext{
${ }^{266}$ Vgl. dazu Gliederungspunkt 2.2.3.3.2.1.

${ }^{267}$ Vgl. dazu LEOPOLDSBERGER/THOMAS/NAUBEREIT (2016), S. 444, LINDMAYER/DIETZ (2018), S. 287.

${ }^{268} \mathrm{Zu}$ derartigen Komplexitätsproblemen vgl. HERING (2017), S. 149.
} 
Geldnot oder Unverständnis abgelehnt oder vertagt, sind große Instandhaltungsmaßnahmen und Sonderumlagen absehbar. Bei Vorhandensein derartiger Warnzeichen stellt sich jedoch die Frage, ob man solche Investitionsobjekte nicht von vorneherein konsequent aus seinen Überlegungen ausschließen sollte. Zumindest darf bezweifelt werden, daß eine treffende Einschätzung der anderen Eigentümer selbst bei eingehender Analyse möglich ist. Anstatt einer solchen ist eine Orientierung an der Peters'schen Formel ${ }^{269}$ oder an Mittelwerten aus der Zweiten Berechnungsverordnung ${ }^{270}$ besser geeignet. ${ }^{271}$ Solche Mittelwerte erscheinen auch daher angebracht, weil zumindest im theoretischen Idealfall die Beitragspflicht zur Rücklage langfristig den Instandhaltungskosten entspricht. Mindestens führt das Ansparen der Instandhaltungsrücklage zu einer periodischen Glättung, welche größere Sprünge im Zeitverlauf theoretisch ausgleicht. ${ }^{272}$

Obwohl jedes Modellergebnis im Einzelfall ganz wesentlich von der Qualität seiner Eingangsdaten abhängt, muß der Investor der Komplexitätsexplosion Einhalt gebieten. Da in der langfristigen Betrachtung nur die Summe tatsächlicher Kosten relevant ist, können (und sollten) vergröbernde Vereinfachungen durchaus vorgenommen werden, um von der detaillierten, formellen Ebene zu abstrahieren. Fundierte Kenntnisse des Rechenwerks empfehlen sich dennoch, weil man aus den Daten durchaus Rückschlüsse auf Qualifikation der Verwaltung und charakterliche Eigenschaften der übrigen Eigentümer ziehen und diese bei der Vorselektion potentieller Investitionsobjekte nutzen kann.

\subsection{Berücksichtigung des ausgeprägten Unikatscharakters von} Eigentumswohnungen

\subsection{Nutzwertanalyse zur Anpassung von Durchschnittswerten an den Einzelfall}

Die vorherigen Ausführungen unterstreichen ein wesentliches Spannungsfeld: Weder aus möglichst ähnlichen Objekten noch aus dem Bewertungsobjekt selbst kann man genaue Eingangsdaten gewinnen. Weil jede Eigentumswohnung ein Unikat ist, beeinflussen einerseits Standort und Eigentümergemeinschaft viele wichtige Faktoren des Gebäudes. Andererseits mißlingt die Schätzung detaillierter Prognosewerte hinsichtlich künftiger Instandhaltungskosten auf Basis von gebäudebezogenen Vergangenheitsdaten, weil frühere Investitionen weder mit heutiger Technik noch mit heutigen Preisen vergleichbar sind. Der Instandhaltungsbedarf steigt mit zunehmendem Gebäudealter wenn auch nicht linear. ${ }^{273}$ Beispielsweise kann der genaue Zeitpunkt der Erneuerung einer Heizungsanlage kaum vorhergesehen werden, zudem fällt eine große Auszahlung

${ }^{269}$ Vgl. PETERS (1984).

${ }^{270}$ Vgl. § 28 II. BV.

${ }^{271}$ Mangels Alternativen werden diese Verfahren in der Literatur oft zur Schätzung der Beitragspflicht zur Instandhaltungsrücklage empfohlen, vgl. TOLL/WALOCHNIK (2013), S. 24, ENGELHARDT (2017a), Rn. 52, MUSIELACK (2017), S. 81, LINDMAYER/DIETZ (2018), S. 286, HÜGEL (2019b), Rn. 12.

${ }^{272}$ Auch zum Folgenden vgl. LEOPOLDSBERGER/THOMAS/NAUBEREIT (2016), S. $444 \mathrm{f}$.

${ }^{273}$ Vgl. dazu KURZROCK (2017a), S. 423, HERR (2017), S. 434. 
auf einmal an. Gleiches gilt für das Mietverhältnis: Auch vergangene Mietzahlungen derselben Eigentumswohnung geben nur bedingt Aufschluß darüber, welche Einnahmen man zukünftig erwarten kann. ${ }^{274}$ Es ist denkbar, daß der bisherige Mietpreis aus Vermietersicht unvorteilhaft ausgehandelt wurde und deutlich unter der potentiellen Zahlungsbereitschaft der Mieter lag.

Der Versuch, Daten aus möglichst ähnlichen Objekten zu gewinnen, scheitert an der fehlenden Vergleichbarkeit. ${ }^{275}$ Durchschnittswerte aus Mietrichtwert-Tabellen beziehen sich oft auf eine ganze (Klein-) Stadt oder Region. ${ }^{276}$ Mietspiegel aggregieren Wohnraum zu Klassen wie z.B. mittlere Lage, 50-70 qm, Baujahr 1970-1980. Diese sind sehr grob und beinhalten Daten vieler heterogener Vergleichswohnungen, deren Vergleichbarkeit man in Frage stellen darf. ${ }^{277}$ Es sollte bezweifelt werden, ob die Lebensqualität in einer heruntergekommenen Nebenstraße im Innenstadtbereich wirklich besser ist und einen höheren Mietpreis rechtfertigt als auf einer ruhigen Nebenstraße in dörflicher Umgebung mit dennoch guter Verkehrsanbindung. Außerdem entspringen Mietspiegel zeitintensiven, großflächigen Erhebungen von Mieterbund und Grundbesitzerverein. Aufgrund des Erhebungsaufwands sind die zusammengetragenen Daten meist zwei bis vier Jahre alt und selbst im Zeitpunkt der Veröffentlichung nicht mehr aktuell. ${ }^{278}$ Überdies sind die Konditionen einer Neuvermietung auch von den Vorlieben genau derjenigen Mietinteressenten abhängig, die zu diesem Zeitpunkt eine Wohnung suchen. ${ }^{279}$

Das Zusammentragen von Vergangenheits- und Erfahrungsdaten sowie deren Hochrechnung zu durchschnittlichen Zahlungsströmen ist dennoch ein sinnvoller erster Schritt. Oftmals bleibt dem Bewertungssubjekt mangels Alternative nichts anderes übrig, als sich auf vermeintlich durchschnittliche Daten zu stützen. Der ausgeprägte Unikatscharakter einer Eigentumswohnung erschwert deren Anwendbarkeit. Die Erfordernisse des Einzelfalls werden nicht ausreichend gewürdigt, wenn man Erfahrungswerte mit Vergangenheitsbezug oder Durchschnittswerte heranzieht, die überdies für einen ganzen Ort (-steil) gelten sollen. Ganz allgemein ist die Anwendbarkeit von Durchschnittswerten „,von zahlreichen Wenn und Aber abhängig.“"280

\footnotetext{
${ }^{274}$ Zum Folgenden vgl. ROPETER (1998), S. 253 ff., SCHULTE/SOTELO/ALLENDORF/ROPETER-AHLERS/ $\operatorname{LANG}$ (2016), S. 587.

275 Ähnlich OLBRICH (2003), S. 348, HOMANN (2017), S. 303, BRAUER (2019b), S. 409.

${ }^{276} \mathrm{Vgl}$. HAUS UND GRUND RHEINLAND (2014), S. 13. Auch die statistischen Verfahren zur Erstellung von Mietspiegeln variieren von Kommune zu Kommune, vgl. VOIGTLÄNDER (2019), S. 150 f.

${ }^{277}$ Vgl. SCHLITTGEN (2017), S. 154 f. „Es stellt sich die Frage, ob gemäß der Wohnlagenkarte zum Beispiel die mittlere Wohnlage im Bezirk Mitte wirklich vergleichbar ist mit der mittleren Wohnlage in Außenbezirken von Berlin, wie zum Beispiel Marzahn-Hellersdorf im Osten der Stadt, so wie es der Mietspiegel unterstellt.“ KAUERMANN/WINDMANN (2016), S. 213.

${ }^{278}$ Vgl. FUCHS (2017), S. 77.

279 Vgl. TILMES/JAKOB/PITSCHKE (2016), S. 900.

${ }^{280}$ SCHMALENBACH (1966), S. 48.
} 
Die quantitative Investitionstheorie interessiert sich durchaus für qualitative Faktoren wie Fassade, Gebäudetechnik und Dachabdichtung, weil sie die zu erzielenden Zukunftserfolge und damit den Bewertungsgegenstand beeinflussen. Sei es die schöne Aussicht vom Balkon, die sich positiv auf die erwartete Miethöhe auswirkt, oder die verrostete alte Heizungsanlage, die sich negativ in Form erwarteter Instandhaltungskosten niederschlägt. Der Einmaligkeit der Entscheidungssituation und der Länge des Planungshorizonts muß durch Anpassung durchschnittlicher Zahlungsströme an die vorliegenden Gegebenheiten Rechnung getragen werden, wobei derartige qualitative Faktoren berücksichtigt werden müssen. Dabei kann eine Nutzwertanalyse behilflich sein. ${ }^{281}$ Man verwendet sie üblicherweise für den Vergleich bzw. die Auswahl zwischen verschiedenen Handlungsalternativen, deren Merkmale man nur unzureichend quantitativ messen kann. ${ }^{282}$ Zunächst gewichtet man entsprechend der eigenen, subjektiven Einschätzung die Relevanz der verschiedenen Merkmale potentieller Bewertungsobjekte. ${ }^{283}$ Danach vergibt man Punkte in Abhängigkeit davon, wie die Merkmalsausprägungen den eigenen, subjektiven Präferenzen entsprechen. Das Objekt, dem die meisten Punkte zugeordnet werden, erscheint am gefälligsten.

Eine Nutzwertanalyse ist auch dann anwendbar, wenn nur eine einzige Eigentumswohnung hinsichtlich ihrer Vorteilhaftigkeit beurteilt werden soll. ${ }^{284}$ Gedanklicher Ausgangspunkt ist eine durchschnittliche Eigentumswohnung als fiktives Vergleichsobjekt, welches Gegenstand von Mietrichtwerttabellen und weiteren Durchschnittswerten ist. ${ }^{285}$ Sein Nutzwert sei das arithmetische Mittel aller erreichbaren Nutzwerte. In der hier vorzustellenden Form führt eine Nutzwertanalyse zu Werten zwischen null und 200 Prozent, je nachdem, wie die Eigentumswohnung aus Sicht des Bewertungssubjekts im Vergleich zu einer fiktiven durchschnittlichen Wohnung wahrgenommen wird. In Abhängigkeit davon, welchen Nutzwert das Bewertungsobjekt erreicht, gelingt es, Durchschnittswerte wie die ortsübliche Miete oder Instandhaltungskostenpauschalen an die vorliegende Eigentumswohnung anzupassen, indem solche durchschnittlichen Zahlungsströme nach oben oder unten adjustiert werden. Gebäudeindividuelle Faktoren werden somit indirekt erfaßt und in die Bewertung einbezogen.

${ }^{281}$ Zur Nutzwertanalyse vgl. DREYER (1975), BECHMANN (1978), ZANGEMEISTER (2014).

${ }^{282}$ Vgl. MENSCH (2002), S. 212 ff., BLOHM/LÜDER/SCHAEFER (2012), S. 151.

${ }^{283}$ Zur Vorgehensweise der Nutzwertanalyse vgl. ADAM (2000), S. 93 ff., GÖTZE (2014), S. 196, BUSSE VON COLBE/WITTE (2018), S. $307 \mathrm{ff}$.

${ }^{284}$ Zur folgenden Vorgehensweise vgl. TOLL/WALOCHNIK (2013), S. 22 f. Ein ähnliches Punktemodell nutzen SCHARMANSKI/WIENCKE (2017), S. 729 ff., zur Analyse des Makrostandorts. Zur Nutzwertanalyse im Rahmen von Immobilienanalysen vgl. FELDMANN/GERSTNER/HOFMANN/ISENHÄFER/ SEGERER/V̈̈TH (2016), S. 403 f., KURZROCK (2017b), S. 729 f.

${ }^{285}$ Obwohl die Nutzwertanalyse üblicherweise für Auswahlentscheidungen verwendet wird, findet sich ein ähnlicher Vorschlag der Nutzwertanalyse für nur ein Objekt, das ,einer hypothetischen Alternative gegenübergestellt" wird, bei ZANGEMEISTER (2014), S. 46. 
Die ausgeprägte Subjektivität der Methode ist höchst nützlich. Man bedenke, daß auf dem unvollkommenen Immobilienmarkt viele Anbieter und Nachfrager mit unterschiedlichen Präferenzen aufeinandertreffen. ${ }^{286}$ Schließlich beruht jede Bewertung auf subjektiven Prognosen der Zukunft und individueller Würdigung des Einzelfalls. Es wäre sehr gewagt, vermeintlich objektive Eingangsdaten (z.B. auf Basis eines Mietspiegels) unreflektiert zu übernehmen und in die Zukunft hochzurechnen. Die Nutzwertanalyse stellt ein Werkzeug dar, dem unvollkommenen Immobilienmarkt zu begegnen und Entscheidungen bestmöglich vorzubereiten. ${ }^{287}$

\subsection{Angemessenheit kategoriebezogener Nutzwerte aufgrund juristischer Doppelstellung des Vermieters}

Es liegt auf der Hand, daß sich verschiedene Faktoren unterschiedlich stark auf den von der WEG erzeugten Aufwand und die Vermietbarkeit auswirken. Es bietet sich an, beiden Einflußbereichen Rechnung zu tragen. Sinnvoll erscheint die Klassifizierung von Immobilienmerkmalen hinsichtlich dessen, ob sie in den Augen des Investors nur die WEG, nur das Mietverhältnis oder beide Sphären beeinflussen. Der vermeintliche Umweg über Kategorien erlaubt die gezielte Ermittlung mehrerer Nutzwerte, und einzelfallbezogene Aspekte werden besser berücksichtigt. Die Berechnung von kategoriebezogenen Nutzwerten führt zu mehr Transparenz und Anschaulichkeit, wenn z.B. eine Kategorie Bauqualität einen überdurchschnittlichen Wert annimmt, während eine Kategorie Ausstattung dürftig ausfällt. Sie ermöglicht auch eine differenzierte Hochrechnung

${ }^{286}$ Vgl. ROTTKE/KRAUTZ (2017), S. 783 ff. Umsicht ist zudem bei jeder Form von Punktbewertungsmodellen geboten, denn man mißt ,nicht die Realität, sondern die empfundene Differenz zwischen einer subjektiv wahrgenommenen Realität und einer subjektiven, nicht offengelegten Idealvorstellung“, $\operatorname{ADAM}(1996)$, S. 83.

${ }^{287}$ Überdies stiftet die Nutzwertanalyse bereits bei der Suche und Vorselektion geeigneter Investitionsobjekte einen wertvollen Beitrag. Eine Eigentumswohnung kann dem Investor aufgrund eines überforderten Verwalters, Blockadehaltungen oder Streitigkeiten der Eigentümer untereinander viel Leid bescheren, selbst wenn sie aus quantitativer Sicht rentabel sein mag. Eine Definition von Ausschlußkriterien ist denkbar, bspw. eine nahezu leere Rücklage, das Vorhandensein eines Mehrheitseigentümers mit mehr als 50 Prozent der Stimmrechte oder Streitigkeiten innerhalb der WEG, erkennbar in alten Versammlungsprotokollen. Vereinzelt findet man Eigentümer, die das Wohnungseigentumsrecht nicht verstehen (wollen), weil es ihren Gerechtigkeitsvorstellungen (bzw. finanziellen Interessen) widerspricht, vgl. DRASDO (2017a), Rn. 111 und Rn. 115, SCHWERING (2019), S. 291 f. Allein im Jahr 2017 wurden vor deutschen Amtsgerichten 22.479 Verfahren über WEG-Binnenstreitigkeiten ausgetragen, vgl. STATISTISCHES BUNDESAMT (2018c), S. 38. Zudem wurden 3.066 Verfahren vor einem Landgericht als Berufungsinstanz ausgetragen, vgl. STATISTISCHES BUNDESAMT (2018c), S. 68. Neben vielen sehr angenehmen Gemeinschaften hat der Verfasser in seiner bisherigen Berufserfahrung auch solche erlebt, die aus (vermeidbarer) Geldnot Entscheidungen so lange hinausgezögert haben, bis die Instandsetzungsarbeiten aufgrund von Folgeschäden deutlich teurer wurden. Andererseits existieren viele sehr angenehme Eigentümergemeinschaften mit kooperativem und freundschaftlichem Klima, die ein harmonisches Miteinander pflegen und Probleme schnell und unkompliziert lösen. Zur qualitativen Immobilienvorselektion anhand von Standort-, Objekt- und Mietanalysen, vgl. FELDMANN/GERSTNER/HOFMANN/ISENHÄFER/SEGERER/VÄTH (2016), S. 412, ERTLE-STRAUB (2019), S. $372 \mathrm{ff}$. 
ortsüblicher Mieten einerseits und durchschnittlicher Instandhaltungskosten andererseits.

Innerhalb der WEG wirken sich Stimmrecht, Wesenszüge und Kooperationsbereitschaft der Miteigentümer auf nicht umlagefähige Nebenkosten aus. ${ }^{288}$ Der Investor muß sich zwangsläufig an Gemeinschaftskosten beteiligen, die von Rechtsstreitigkeiten und Instandhaltungsmaßnahmen hervorgerufen werden. Im Zusammenspiel mit einer unprofessionellen Verwaltung können Blockadehaltungen anderer Eigentümer vermeidbare Zusatzkosten hervorrufen, wenn der Investor die Kosten sinnloser Rechtsstreitigkeiten mittragen muß oder auf Schäden am Gemeinschaftseigentum nicht so schnell reagieren kann, wie er möchte. Sofern eine Datenbeschaffung überhaupt möglich ist, erfordert eine richtige Deutung von vergangenen Versammlungsprotokollen natürlich große Menschenkenntnis und Erfahrung. Dennoch gibt es eine Reihe von Indizien, die für eine problematische Eigentümergemeinschaft oder Verwaltung sprechen können - aber nicht müssen: Dazu gehören z.B. späte Jahresabrechnungen oder häufige bzw. zeitintensive Eigentümerversammlungen, die sich mit Themen wie Versicherungsschäden, Instandhaltungsmaßnahmen, Hausgeldrückständen oder juristischen Konflikten befassen. Findet die Eigentümerversammlung erst in der zweiten Jahreshälfte statt oder werden bestimmte Themen wiederholt ergebnislos behandelt, kann die Handlungsfähigkeit der WEG gelähmt sein.

Meistens wird die Vermarktungsfähigkeit gegenüber Mietern und somit die Höhe der erwarteten Mieteinnahmen von solchen Unwägbarkeiten innerhalb der WEG nicht berührt. ${ }^{289}$ Häufig üben völlig andere Faktoren Einfluß auf die Vermietbarkeit der Wohnung aus, bspw. Ausstattung, Lage, Zuschnitt, Aussicht oder Verkehrsanbindung. ${ }^{290}$

Selbstverständlich gibt es auch Kriterien, welche nicht trennscharf einer der beiden Sphären zugeordnet werden können. Man denke an Folgeschäden im Sondereigentum, der durch Sanierungsstau im Gemeinschaftseigentum ausgelöst wird. Dann steht zu befürchten, daß dem Mieter Minderungsrechte zustehen, obwohl der Vermieter nicht zur

${ }^{288}$ Zur WEG als Zufalls- und Schicksalsgemeinschaft vgl. DRASDO (2017a), Rn. $111 \mathrm{ff}$. Zur Relevanz einer guten Nachbarschaft und deren starken Auswirkungen auf Lebensqualität, Wohnzufriedenheit und Immobilienwert vgl. KEMPER/SCHÖFFEL (2014), S. 203.

${ }^{289}$ Dies gilt zumindest, sofern der Streitgegenstand innerhalb der WEG kein Schaden im Gemeinschaftseigentum ist, der das Sondereigentum beeinträchtigt.

${ }^{290} \mathrm{Vgl}$. FELDMANN/GERSTNER/HOFMANN/ISENHÄFER/SEGERER/VÄTH (2016), S. 390, SCHARMANSKI/ WIENCKE (2017), S. 736, FESSELMANN/KOBABE/SCHWENZFEIER/SIEGEMUND (2018), S. 6. „Immer wieder wird behauptet, dass es bei einer selbstgenutzten Immobilie nur darauf ankomme, dass Sie [sic!] Ihnen persönlich gefällt [...]. Dabei wird nämlich der entscheidende Aspekt übersehen, dass der Zweck eines Immobilienerwerbs sich ändern kann. [Es ist] denkbar, dass Sie eine zunächst für die Eigennutzung gekaufte Immobilie zu einem späteren Zeitpunkt vermieten oder verkaufen wollen. Spätestens dann holt Sie die Frage ein, ob die Immobilie sich auch für einen Durchschnittsnutzer eignet und entsprechend hohe Mieteinnahmen oder Verkaufspreise erzielen kann." RENNERT (2012), S. 4. 
Abhilfe berechtigt ist. ${ }^{291}$ Sofern sich die WEG nicht gerade um solche Schäden streitet, sondern Beschlußanfechtungsverfahren führt, ${ }^{292}$ sich über Verwalterbestellung, Jahresabrechnung oder Beiratswahl streitet oder gerichtlich die Frage nach der Anzahl der vom Verwalter einzuholenden Handwerkerangebote austrägt, ${ }^{293}$ nimmt der Mieter Probleme innerhalb der WEG oft gar nicht wahr. ${ }^{294}$ Die Anzahl der Berührungspunkte hängt vom Einzelfall $\mathrm{ab}$.

\subsection{Vorgehensweise der Nutzwertanalyse}

Im ersten Schritt der Nutzwertanalyse muß das Bewertungssubjekt sich Klarheit verschaffen, welche Merkmale einer Eigentumswohnung aus seiner Sicht relevant sind. ${ }^{295}$ Bedeutsame Faktoren können z.B. Parkmöglichkeiten, Verkehrsanbindung oder der Zuschnitt der Wohnung sein. ${ }^{296}$ Die relevant erscheinenden Merkmale werden zu unterschiedlichen Kategorien. $\mathrm{\iota}=(1,2, \ldots, \vartheta)$ zusammengefaßt, innerhalb derer die Kriterien als $\mathrm{K}^{\mathrm{\iota}}=\left(1^{\iota}, 2^{\iota}, \ldots, \mathrm{r}^{\mathrm{\iota}}\right)$ bezeichnet werden. Folgende Kategorisierung erscheint sinnvoll:

- Kategorien, die nur die Vermietbarkeit beeinflussen,

- solche, die nur auf nicht umlagefähige Nebenkosten wirken, sowie

${ }^{291}$ Ein trauriges Beispiel für langfristigen Mietausfall liefert die Besprechung des BGH-Urteils V ZR 101/16 von ELZER (2018c), S. 293: Innerhalb einer Sondereigentumseinheit traten feuchtigkeitsbedingte Schimmelschäden auf. Nicht nur ein Beschluß für die Instandsetzung, sondern bereits für die Ursachensuche wurde mehrfach zurückgestellt und von einer Eigentümerversammlung zur nächsten vertagt - diese fand selbstredend jeweils im Folgejahr statt. Nach vielen Jahren wurde die WEG zur Zahlung von Schadenersatz verurteilt.

${ }^{292}$ Diese machen einen sehr großen Prozentsatz der Rechtsstreitigkeiten innerhalb von Eigentümergemeinschaften aus, vgl. SAUREN (2014g), Rn. 17. Zur Beschlußanfechtung vgl. ROTH (2018), MERLE (2018b).

${ }^{293}$ Zum „Sinn und Unsinn der Forderung nach drei Vergleichsangeboten“ vgl. CASSER (2018), zum „Drei-Angebots-Modell im Wohnungseigentum“vgl. DRASDO (2018a).

${ }^{294}$ Er beschwert sich allenfalls über eine späte Abrechnung. Schließlich ist der Vermieter zur Erstellung der Betriebskostenabrechnung auf Daten der WEG-Jahresabrechnung angewiesen. Zu diesem Problemfeld vgl. BLANK (2004), MÜLLER (2015e), Rn. 255, SUILMANN (2017c). Der Vermieter muß die Betriebskosten immerhin erst innerhalb eines Jahres nach Ende der Abrechnungsperiode erstellen, vgl. § 556 Abs. 3 BGB, vgl. dazu BUSCH (2017a), S. 695, PFEIFER (2019a), Rn. 1233 ff. Wird eine Pauschalmiete einschließlich umlagefähiger Nebenkosten vereinbart, erübrigt sich diese Schnittstelle ganz, vgl. § 565 Abs. 1 BGB, vgl. dazu auch PFEIFER (2019a), Rn. 275 ff.

${ }^{295}$ Zur folgenden Vorgehensweise vgl. TOLL/WALOCHNIK (2013), S. 22 f., dort ohne Kategorisierung. Zur Vorgehensweise der Nutzwertanalyse und ihren Einzelschritten im Allgemeinen vgl. BECHMANN (1978), S. 26 ff., ADAM (2000), S. 93 ff., ZANGEMEISTER (2014), S. 69 ff., BUSSE VON COLBE/WITTE (2018), S. 313 ff. In der Literatur weichen Anzahl und Inhalt der Schritte zum Teil voneinander ab.

${ }^{296}$ Welche Kriterien relevant sind, ist subjektiv und situationsabhängig. Zur Generierung von Ideen findet man in der Literatur unterschiedliche Listen, deren Determinanten bei einer Immobilienanalyse wertvoll sein können, siehe z.B. FAUST (2009), S. 64 ff., HAAS (2010), S. 46, KELLER (2013b), S. 25 f., STÜRZER/KOCH/HOPFENSPERGER/STERNS/STERNS-KOLBECK/ZIEGELMAYER (2016), S. 339 ff., FELDMANN/GERSTNER/HOFMANN/ISENHÄFER/SEGERER/VÄTH (2016), S. 386 f., SCHARMANSKI/ WIENCKE (2017), S. 736 f., KURZROCK (2017b), S. 736 ff. Insbesondere zu Risikofaktoren vgl. SCHÄFERS/ WURSTBAUER (2016), S. $1040 \mathrm{ff}$. 
- Kategorien, die für die Berechnung beider Nutzwerte relevant sind.

Bildet man z.B. eine Kategorie Eigentümergemeinschaft, kann angenommen werden, daß alle enthaltenen Kriterien lediglich auf den Nutzwert hinsichtlich der nicht umlagefähigen Nebenkosten $\mathrm{NW}_{\mathrm{NUNK}}$ wirken, während z.B. die Kriterien einer Kategorie Makro-Standortfaktoren ausschließlich auf den Nutzwert der Miethöhe $\mathrm{NW}_{\mathrm{MH}}$ wirken dürften. Die Kategorie Erneuerungsbedarf hingegen dürfte beide Nutzwerte gleichermaßen beeinflussen. ${ }^{297}$

Verzerrungen durch Doppelerfassungen sollten vermieden werden. Daher sollten bereits bei der Bildung von Kategorien Überlegungen zum Einfluß der Merkmale angestellt und auf eine eindeutige Zuordnung jedes Kriteriums zu genau einer Kategorie geachtet werden. Im zweiten Schritt ordnet man den Kriterien $\mathrm{K}^{\mathrm{l}}$ Gewichtungsfaktoren GEW $_{\mathrm{K}^{\mathrm{L}}}$ entsprechend ihrer subjektiv empfundenen Relevanz zu. Diese sollen zeigen, wie wichtig ein Teilaspekt aus subjektiver Sicht wahrgenommen wird. Empfehlenswert ist eine Skala von null (für völlig unbedeutend, z.B. Farbe der Dachziegel) bis vier (für wichtige Schlüsselkriterien, z.B. Parkmöglichkeiten). Um die Beurteilung nicht durch Einflüsse vor Ort zu verfälschen, erscheint es ratsam, relevante Teilaspekte vor dem ersten Besichtigungstermin - oder noch vor Beginn der Suche - zu erfassen und zu gewichten. Im dritten Schritt wird den Merkmalsausprägungen ein Zielerfüllungsgrad ZEG $\mathrm{K}_{\mathrm{K}^{\iota}}$ Zugeordnet. Entsprechend deren Wahrnehmung beim Besichtigungstermin besagt dieser, wie stark sich die jeweilige Merkmalsausprägung mit den Präferenzen des Entscheiders deckt. Auch hier bietet sich eine Skala zwischen null (für eine absolute Zielverfehlung) und vier (für eine überdurchschnittliche Zielerfüllung) an. Im vierten Schritt wird für jedes Kriterium $\mathrm{K}^{\mathrm{l}}$ der jeweilige Teilnutzwert $\mathrm{TNW}_{\mathrm{K}^{\mathrm{\iota}}}$ errechnet, indem sein Zielerfüllungsgrad $Z_{E G_{K^{\iota}}}$ mit seiner Gewichtung $\mathrm{GEW}_{\mathrm{K}^{\iota}}$ multipliziert und anschließend durch die Summe aller Gewichte der Kategorie $G_{S U M}^{\iota}$ dividiert wird. Der Nutzwert der jeweiligen Kategorie NW ${ }^{\mathrm{l}}$ wird schließlich im fünften Schritt ermittelt, indem die Teilnutzwerte aller Kriterien einer Kategorie summiert werden. ${ }^{298}$ Tabelle 6 gibt die skizzierte Vorgehensweise zur Errechnung der Kategorie-Nutzwerte wieder.

${ }^{297}$ Beispielsweise wirken Instandhaltungsmaßnahmen sowohl auf die Finanzlage der Wohnungseigentümergemeinschaft als auch auf die Vermietbarkeit z.B. die wahrgenommene Gebäude- bzw. Wohnqualität, die für einen langfristigen Erhalt der Vermietbarkeit wichtig ist, vgl. MARKMANN/ MARKMANN/ROTTKE (2017), S. 647.

${ }^{298}$ Kritisch äußern sich BUSSE VON COLBE/WITTE (2018), S. 321: „Unabhängig [von der] Aggregationsvorschrift $[\ldots]$ erweist es sich als methodisch problematisch, ordinal skalierte Daten analog kardinal skalierter Daten zu behandeln. Im Rahmen der Aggregation wird implizit unterstellt, dass sich der Abstand der ordinal skalierten Daten bzw. Klassengrenzen sachlich interpretieren lässt. Diese implizite Annahme ist theoretisch nicht haltbar.“ 


\begin{tabular}{|c|c|c|c|}
\hline $\begin{array}{l}\text { Kriterium } \mathbf{K}^{\mathbf{l}} \\
=\left(\mathbf{1}^{\mathbf{l}}, \ldots, \mathbf{r}^{\mathbf{l}}\right)\end{array}$ & $\begin{array}{l}\text { Gewichtung von } \\
\mathbf{K}^{\mathbf{l}}\left(\mathbf{G E W}_{\mathbf{K}^{\mathbf{l}}}\right) \\
0=\text { irrelevant bis } \\
4 \text { = sehr wichtig }\end{array}$ & 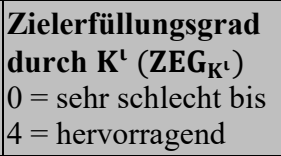 & $\begin{array}{l}\text { Teilnutzwert von } \\
\mathbf{K}^{\mathbf{l}}\left(\mathbf{T N W}_{\mathbf{K}^{\mathbf{l}}}\right)\end{array}$ \\
\hline $1^{\iota}$ & $\mathrm{GEW}_{1} \mathrm{\iota}$ & $Z_{E G}$ & $\mathrm{TNW}_{1^{\mathrm{\iota}}}=\frac{\mathrm{GEW}_{1^{\mathrm{\iota}}}}{\mathrm{G}_{\text {sum }}^{\mathrm{L}}} \cdot \mathrm{ZEG}_{1^{\mathrm{\iota}}}$ \\
\hline$\ldots$ & $\ldots$ & $\ldots$ & $\ldots$ \\
\hline \multirow[t]{2}{*}{$r^{l}$} & $\mathrm{GEW}_{\mathrm{r}^{\mathrm{t}}}$ & $\mathrm{ZEG}_{\mathrm{r}^{\mathrm{t}}}$ & $\mathrm{TNW}_{\mathrm{r}^{\mathrm{t}}}=\frac{\mathrm{GEW}_{\mathrm{r}^{\mathrm{l}}}}{\mathrm{G}_{\mathrm{sum}}^{\mathrm{l}}} \cdot \mathrm{ZEG}_{\mathrm{r}^{\mathrm{l}}}$ \\
\hline & $\begin{array}{l}\text { Gewichtungssumme } \\
\text { der Kategorie } \mathrm{\imath} \\
\mathrm{G}_{\text {sum }}^{\mathrm{\iota}}=\sum_{\mathrm{K}=1^{\mathrm{\iota}}}^{\mathrm{r}^{\mathrm{\iota}}} \mathrm{GEW}_{\mathrm{K}^{\mathrm{\iota}}}\end{array}$ & & $\begin{array}{l}\text { Nutzwert der Kategorie } \mathrm{\iota} \\
\mathrm{NW}^{\mathrm{l}}=\sum_{\mathrm{K}=1^{\mathrm{\iota}}}^{\mathrm{r}^{\mathrm{l}}} \mathrm{TNW}_{\mathrm{K}} \\
\text { mit } 0 \leq \mathrm{NW}^{\mathrm{l}} \leq 4\end{array}$ \\
\hline
\end{tabular}

Tabelle 6: Berechnung des Kategorie-Nutzwerts

Um dem Einfluß beider Rechtssphären - Vermietung und Wohnungseigentümergemeinschaft - gerecht zu werden, sollen auf Basis der verschiedenen Kategorie-Nutzwerte nun unterschiedliche Gesamt-Nutzwerte berechnet und als $\mathrm{NW}_{\mathrm{MH}}$ (Nutzwert Miethöhe) und $\mathrm{NW}_{\mathrm{NUNK}}$ (Nutzwert nicht umlagefähige Nebenkosten) bezeichnet werden. Hierbei ist der in Schritt fünf beschriebenen Vorgehensweise zu folgen, wobei man Kategorien so behandelt wie vormals einzelne Kriterien: Kategoriebezogene Gesamtgewichte $G_{\text {sum }}^{\mathrm{L}}$ stehen nun an der Stelle kriterienbezogener Einzelgewichte $\mathrm{GEW}_{\mathrm{K}^{\mathrm{L}}}$, anstatt des Zielerfüllungsgrades $Z_{E G} G_{K^{\iota}}$ eines Kriteriums setzt man Kategorie-Nutzwerte NW ${ }^{\iota}$ an. Die Vorgehensweise ist in Tabelle 7 dargestellt und führt zum gleichen Ergebnis wie eine kategorielose Berechnung des Gesamtnutzwertes auf direktem Wege.

\begin{tabular}{|c|c|c|c|}
\hline $\begin{array}{l}\text { Kriterium } \mathbf{~} \\
=(1,2, \ldots, \boldsymbol{\vartheta})\end{array}$ & $\begin{array}{l}\text { Gewichtungssumme } \\
\text { Kategorie } \mathbf{l}\left(G_{\text {sum }}^{\mathbf{l}}\right)\end{array}$ & $\begin{array}{l}\text { Nutzwert Katego- } \\
\text { rie } \mathrm{c}\left(\mathrm{NW}^{\mathbf{}}\right)\end{array}$ & $\begin{array}{l}\text { Teilnutzwert Katego- } \\
\text { rie } \iota \text { für Gesamtnutz- } \\
\text { wert }\left(\mathrm{TNW}^{\mathbf{}}\right)\end{array}$ \\
\hline 1 & $\mathrm{G}_{\mathrm{sum}}^{1}$ & $\mathrm{NW}^{1}$ & $\mathrm{TNW}^{1}=\frac{\mathrm{G}_{\text {sum }}^{1}}{\mathrm{G}_{\text {sum }}} \cdot \mathrm{NW}^{1}$ \\
\hline$\ldots$ & $\ldots$ & $\ldots$ & $\ldots$ \\
\hline \multirow[t]{2}{*}{$\vartheta$} & $\mathrm{G}_{\text {sum }}^{\vartheta}$ & $\mathrm{NW}^{\vartheta}$ & $\mathrm{TNW}^{\vartheta}=\frac{\mathrm{G}_{\mathrm{sum}}^{\vartheta}}{\mathrm{G}_{\mathrm{sum}}} \cdot \mathrm{NW}^{\vartheta}$ \\
\hline & $\begin{array}{l}\text { Gewichtungssumme } \\
\mathrm{G}_{\text {Sum }}=\sum_{\mathrm{t}=1}^{\vartheta} \mathrm{G}_{\text {sum }}^{\mathrm{t}}\end{array}$ & & $\begin{array}{l}\text { Gesamtnutzwert } \\
\mathrm{NW}=\sum_{\mathrm{l}=1}^{\vartheta} \mathrm{TNW}^{\mathrm{\iota}} \\
\text { mit } 0 \leq \mathrm{NW} \leq 4\end{array}$ \\
\hline
\end{tabular}

Tabelle 7: Berechnung des Gesamtnutzwerts 
Nachdem die zwischen null und vier skalierten Nutzwerte $\mathrm{NW}_{\mathrm{MH}}$ und $\mathrm{NW}_{\mathrm{NUNK}}$ berechnet sind, werden diese zu Faktoren weiterverarbeitet, um durchschnittliche Zahlungsströme auf den Einzelfall hochzurechnen, wie Tabelle 8 zeigt.

Mit dem Faktor MH (Miethöhe) können durchschnittliche Mietpreise einer Ortschaft auf die individuellen Gegebenheiten umgerechnet werden, wobei der Wertebereich hier durch Skalierung auf 80 bis 120 Prozent des Durchschnittswerts begrenzt ist.

Die nun individualisierten Mieteinnahmen müssen anschließend mit dem Faktor MOL (Monate ohne Leerstand) multipliziert werden, dessen Normierung zu erwarteten Einnahmen zwischen null und zwölf Monatsmieten pro Jahr führt. Im Fall von Leerstand fallen sie zeitweise aus. Da dann auch der umlagefähige Anteil des Hausgelds vom Eigentümer aufgebracht werden muß, ist die monatliche Höhe des Hausgelds mit dem Faktor (12./. MOL) zu multiplizieren. ${ }^{299}$

Durchschnittliche Instandhaltungs- und Rechtsanwaltskosten können mit dem Faktor NUNK (nicht umlagefähige Nebenkosten) umgerechnet werden. Es ergeben sich individualisierte, nicht umlagefähige Nebenkosten innerhalb eines Wertebereichs zwischen null und 200 Prozent, bezogen auf Durchschnittswerte. Die Obergrenze von 200 Prozent deutet auf eine abschreckende Eigentümergemeinschaft hin, die doppelt so viele vom Eigentümer zu tragende Nebenkosten erzeugt, als man es von einer typischen WEG erwarten würde. ${ }^{300}$ Die Untergrenze von null Prozent steht für den Entartungsfall, daß die Nachfrage bei einer solch nahezu perfekten Wohnung so hoch ist, daß der Mieter selbst nicht umlagefähige Nebenkosten gerne übernimmt - ein Extremfall aus dem Reich der Theorie.

${ }^{299}$ Allein der Beschluß über den Wirtschaftsplan begründet rechtsverbindlich die Zahlungspflicht des Hausgelds, welches auch bei Leerstand in voller Höhe an die WEG entrichtet werden muß: „Das Nutzungsrisiko liegt beim Eigentümer. Dies gilt selbst dann, wenn die Unbenutzbarkeit einer Wohnung auf Baumaßnahmen beruht, die von der Gemeinschaft veranlasst worden sind." HERMANN (2019b), Rn. 38.

${ }^{300}$ Auch diese Grenzen sind subjektiv und willkürlich. Der aufmerksame Leser mag entgegnen, daß die Grenze bei 200 Prozent sogar noch relativ niedrig ist und daß besonders abschreckende Eigentümergemeinschaften weitaus höhere Kosten mit sich bringen. Dem ist erstens entgegenzuhalten, daß mit Hilfe von Nutzwertanalysen ebenfalls eine Vorauswahl der in Frage kommenden Bewertungsobjekte möglich ist. Mit dieser sollten katastrophale Eigentümergemeinschaften von vorneherein aussortiert werden. Zweitens entscheidet jedes Bewertungssubjekt situationsabhängig, welche Normierung es für sinnvoll und angebracht hält. Für die in Gliederungspunkt 2.3.2.1 vorzustellende Beispielwohnung hält der Verfasser diese Normierung jedenfalls für angemessen. 


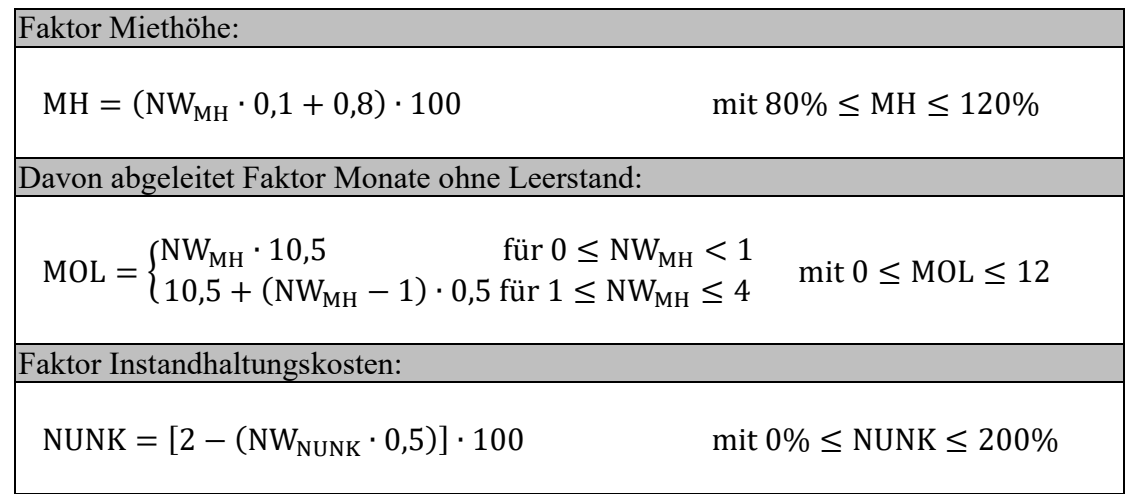

\section{Tabelle 8: Berechnung der Faktoren MH, MOL und NUNK}

\subsection{Zahlungen am Planungshorizont}

Wenn die jährlichen Zahlungsströme quantifiziert werden, sind auch Überlegungen anzustellen, für wie viele Jahre diese Daten anzusetzen sind. Sowohl Unternehmen als auch Immobilien sind Wirtschaftsgüter von sehr langer, nicht aber von unbegrenzter Lebensdauer. ${ }^{301}$ Es gilt, einen Planungshorizont pragmatisch festzusetzen. ${ }^{302}$ Diese Entscheidung ist ex ante immer willkürbehaftet. HERING betont, daß allgemeingültige Empfehlungen nicht möglich sind, hält es aber in vielen Fällen für empfehlenswert, sich an der Laufzeit des längsten verfügbaren Zahlungsstroms zu orientieren. ${ }^{303}$ Es bietet sich für die vorliegende Bewertungssituation an, sich an der erwarteten Lebensdauer der Eigentumswohnung zu orientieren. ${ }^{304}$

Zur Lebensdauer von Gebäuden findet man in der Literatur eine Reihe sehr unterschiedlicher Überlegungen. So spricht HERR von einer achtzigjährigen Nutzungsdauer, ${ }^{305}$ das Steuerrecht unterstellt für Wohngebäude, die nach dem 31.12.1924 fertiggestellt wurden, eine fünfzigjährige Nutzungsdauer. ${ }^{306}$ ROTTKE, EIBEL und KRAUTZ gehen - unter

\footnotetext{
${ }^{301}$ Vgl. BINCKEBANCK/GÖTZEN (2017), S. 928. „Der Planungszeitraum kann allerdings nur in Modellen, nicht aber praktisch bis zum Jüngsten Gericht reichen“, pointiert SCHNEIDER (1992), S. 29.

302 Vgl. SCHLÜCHTERMANN (1996), S. 20 f., HERING (2017), S. 11 f., TOLL/HERING (2017), S. 463. Denn „bei einer ,unendlichen' Unternehmung werden manche Zusammenhänge nicht deutlich, weil sie sich ,im Unendlichen verlieren'. [...] in der Realität ist die Endlichkeit mitunter in wenigen Jahren erreicht“ SIEGEL (1994), S. 3, ähnlich ENGELS (1962), S. 98.

${ }^{303}$ Vgl. HERING (2017), S. 14, ähnlich ROSENBERG (1975), S. 9 f.

${ }^{304}$ Einer ähnlichen Vorgehensweise folgt auch JAPES (2011), S. 49 ff.

${ }^{305}$ Vgl. HERR (2017), S. 434.

${ }^{306}$ Vgl. $\S 7$ Abs. 4 Nr. 2 Buchstabe a EStG.
} 
der Voraussetzung fortlaufender Investitionen - davon aus, daß die technische Lebensdauer von Gebäuden auch jenseits von 100 Jahren liegen kann. ${ }^{307}$ Da man die Lebensdauer einer Eigentumswohnung ex ante einfach nicht kennt, sollen hier die Annahmen der Finanzverwaltung übernommen werden: Angelehnt an die Abschreibungstabellen soll von einer fünfzigjährigen Nutzungsdauer ausgegangen werden. Dies soll keineswegs darüber hinwegtäuschen, daß es sich um einen schablonenartigen Normwert aus dem Bereich des Steuerrechts handelt. ${ }^{308}$ Zum Schutz eines Modells vor Überfrachtung müssen vereinfachende und manchmal auch sehr willkürliche Annahmen getroffen werden.

Innerhalb dieser fünfzigjährigen Lebensdauer wird angenommen, daß aus der Eigentumswohnung ein Zahlungsstrom in jährlich konstanter Höhe gewonnen werden kann. Am Ende des Planungshorizonts können weitere Zahlungen auftreten, die ebenfalls in die Überlegungen einzubeziehen sind. Einzelfallabhängig kann es sich um Erhaltungskosten oder Verkaufserlöse handeln. Die immobilienwirtschaftliche Literatur spricht häufig auch von einem Ende der wirtschaftlichen Nutzbarkeit. ${ }^{309}$ Wenn das Gebäude abgenutzt ist und nicht mehr verkauft werden kann, ist ein Abriß erforderlich, der weitere Kosten nach sich zieht. ${ }^{310}$ Dieser Gedanke soll hier nicht weiter verfolgt werden. Sicherlich gibt es diesen Fall. Andererseits denke man an solche Altbauwohnungen, wie man sie u.a. in Budapest oder Wien findet. Bei entsprechender Pflege, Wartung und Instandhaltung kann eine Immobilie durchaus mehrere 100 Jahre bestehen. ${ }^{311}$ Zusätzlich gilt es zu bedenken, daß die Abrißentscheidung nicht vom Investor allein, sondern von der gesamten Wohnungseigentümergemeinschaft zu treffen ist.

Man könnte auch annehmen, die Eigentumswohnung verfüge am Planungshorizont über eine bestimmte Form von Restwert. Dies kann z.B. die Differenz zwischen Abrißkosten des Gebäudes und Verkaufserlös des Grundstücks sein. SCHULTE ET AL. empfehlen eine auf den Planungshorizont bezogene Verkehrswertermittlung, um das Ergebnis als fiktiven Verkaufserlös zu betrachten. ${ }^{312}$ Dazu müßte man den erzielbaren Preis zu einem bestimmten Zeitpunkt möglichst treffsicher abschätzen. Es wären langfristige Überlegungen über die Entwicklung von Lage und Mietpreis erforderlich. Mit zunehmender Entfernung des Planungshorizonts stellen diese Annahmen immer höhere, zum Teil unerfüllbare Anforderungen, denn die Unsicherheit der Prognose ist im Zeitpunkt $t=n$ am größten. ${ }^{313}$ Ohnehin kann dieses Vorgehen nur dann der Aufgabenstellung entsprechen,

${ }^{307}$ Vgl. ROTTKE/EIBEL/KRAUTZ (2017), S. 12 ff.

${ }^{308}$ Zudem sei angemerkt, daß $§ 7$ Abs. 4 EStG nicht zwischen dem Kauf einer neu errichteten oder einer bestehenden Immobilie differenziert.

${ }^{309}$ Vgl. PFNÜR (2011), S. 98, ROTTKE/EIBEL/KRAUTZ (2017), S. 15, KURZROCK (2017a), S. 430.

${ }^{310} \mathrm{Vgl}$. GROMER (2012), S. 26, ROTTKE/EIBEL/KRAUTZ (2017), S. 11.

${ }^{311} \mathrm{Vgl}$. KURZROCK (2017a), S. 424.

312 Vgl. SCHULTE/SOTELO/ALLENDORF/ROPETER-AHLERS/LANG (2016), S. 590, ähnlich ROPETER(1998), S. $255 \mathrm{f}$.

${ }^{313} \mathrm{Zu}$ diesem Schluß kommen auch die Autoren: „Wenn bereits die Ermittlung eines aktuellen Verkehrswertes mit Unsicherheit behaftet ist, so gilt dies erst recht für die Bestimmung eines künftigen 
wenn ein Verkauf auch vorgesehen ist. Selbst dann erscheint es für den Investor zweckdienlicher, die jenseits des Planungshorizonts erzielbaren Mietüberschüsse als Ertragswert zu berücksichtigen, ,denn bei dem neuen Käufer wird er voraussichtlich dem gleichen Interesse begegnen. "314 Sowohl Käufer als auch Verkäufer stellen Überlegungen zu ihrem jeweiligen Grenzpreis an und orientieren sich dabei am Nutzen der zukünftig erzielbaren Mietüberschüsse. Bei Verkauf gibt der Investor den Zahlungsstrom der Eigentumswohnung ab und erhält im Gegenzug den ausgehandelten Preis. Ein fiktiver Verkaufspreis am Planungshorizont ist somit nichts weiter als ein gedanklicher Umweg, der jedoch mit Nachteilen in Form der Ausblendung subjektiver Faktoren erkauft wird. Man sollte also lieber gleich einen Barwert ansetzen. Wie schon bei der fiktiven Verkehrswertermittlung stellt das Fehlen verwertbarer Eingangsdaten auch hier das entscheidende Hindernis dar.

Analog zu der vermuteten fünfzigjährigen Lebensdauer der Immobilie wird nun angenommen, daß innerhalb dieser Zeit ein uniformer Zahlungsstrom zu erzielen ist, hingegen jenseits von 50 Jahren keinerlei Zahlungen mehr zu erwarten sind. Eine detaillierte Modellierung mehrerer Jahrzehnte widerspricht dem realitätsvereinfachenden Gedanken eines Modells. Weil die von einer Eigentumswohnung hervorgerufenen Geldbewegungen innerhalb der Nutzungsphase mehr oder weniger konstant sind, ${ }^{315}$ bietet sich eine Vereinfachung in Form eines Phasenmodells an, ${ }^{316}$ hierfür spricht auch die Forderung nach Komplexitätsreduktion. Unter einem Phasenmodell versteht man die detaillierte Modellierung der Eingangsdaten für einen ersten Zeitraum, den man als Detailplanungsphase bezeichnet, gefolgt von einer Grobplanungsphase, in der vereinfachte Annahmen getroffen werden.

An welcher Stelle die Grenze zwischen Detail- und Grobplanungsphase zu ziehen ist, hängt vom Einzelfall ab und kann in allgemeiner Form nicht gesagt werden. Hier seien die ersten zehn Jahre explizit betrachtet. Dieser Zeitraum liegt zudem im Rahmen der

Verkehrswertes am Planungshorizont.“ SCHULTE/SOTELO/ALLENDORF/ROPETER-AHLERS/LANG (2016), S. 590. Ohnehin erscheint die Tauglichkeit von Verkehrswertermittlungsverfahren mehr als fragwürdig. Je nach Bewerter und Ermittlungsverfahren können Verkehrswerte mit bis zu 30 Prozent beträchtlich voneinander abweichen, vgl. LEOPOLDSBERGER/THOMAS/NAUBEREIT (2016), S. 431. Zur Tauglichkeit von Verkehrswerten zeigt sich auch WICHMANN irritiert, „denn eigentlich dürfte der ermittelte Verkehrswert nur dann vom Kaufpreis abweichen, wenn bei der zu Grunde liegenden Kaufpreisvereinbarung zumindest ein Marktteilnehmer irrational gehandelt hat. Angesichts der Vielzahl der Fälle, in denen der ermittelte Verkehrswert nicht dem Kaufpreis entspricht, stellt sich die Frage, ob bei Kaufpreisvereinbarungen wirklich so oft Irrationalität im Spiel ist.“ WICHMANN(2017), S. 1.

314 SCHMALENBACH (1917), S. 1, dort in Bezug auf den Wiederverkauf einer „Wirtschaftsanlage“.

${ }^{315}$ Vgl. ROTTKE/KRAUTZ (2017), S. 783 ff., ROTTKE/EIBEL (2017), S. 841. „Zwar stellt die uniforme Ausschüttungsreihe [...] nur eine potentielle Ausschüttungsreihe dar; aber es kann sich aus Vereinfachungsgründen aufdrängen, die tatsächlich erwartete nichtuniforme Ausschüttungsreihe durch eine (fiktive) uniforme Ausschüttungsreihe zu ersetzen“, findet MOXTER(1983), S. 80, ähnlich BUSSE VON COLBE (1957), S. 77.

${ }^{316}$ Auch zum Folgenden vgl. ENGELEITER (1970), S. 72, BALLWIESER (2003), S. 22, WAMELING (2004), S. 69 f., HERING/TOLL (2017b), S. 198. 
gewöhnlichen Zinsbindungsdauer bei Immobilienfinanzierungen. ${ }^{317}$ Die zweite Phase wird oft abgebildet als Barwert einer ewigen Rente, die sich aus unendlich oft wiederkehrenden, gleichbleibenden Zahlungen zusammensetzt. ${ }^{318}$ Dem Ansatz soll hier nicht in dieser Extremform gefolgt werden, weil ein unendlicher Einkommensstrom nicht mit Investitionen in Eigentumswohnungen kompatibel ist. Ihre Nutzungsmöglichkeit stößt trotz langer Lebensdauer irgendwann an Grenzen. Der Barwert einer ewigen Rente hat zudem den Nebeneffekt, daß ausgerechnet der Grobplanungsphase, deren Eingangsdaten sehr vereinfacht abgebildet sind, ein unerwünschtes Übergewicht zukommt. ${ }^{319}$ Dies gilt vor allem bei einem niedrigen Zinsniveau, denn je niedriger der Zins, desto größer der Barwert. Schon bei einem Zinssatz von einem Prozent entspricht die kapitalisierte ewige Rente dem hundertfachen jährlichen Zahlungsstrom. Bei einer zehnjährigen Detailplanungsphase wäre der Anteil am Gesamtwert von rund 90 Prozent unangemessen hoch und völlig verzerrt. Deswegen soll hier zwar von einer langen, aber begrenzten Lebensdauer ausgegangen werden. Es sei von einer zehnjährigen Detailplanungsphase mit einer sich anschließenden 40-jährigen Grobplanungsphase ausgegangen. Da es nun zwei Phasen gibt, ist eine klarstellende Begriffsdefinition erforderlich: Sofern nicht anders genannt, sei im Folgenden mit Planungshorizont das Ende der zehnjährigen Detailplanungsphase bezeichnet.

Nun muß der Barwert der letzten 40 Jahre berechnet und als Einzahlung am Ende des Detailplanungszeitraums erfaßt werden. Zur Berechnung des Barwerts einer endlichen, uniformen Zahlungsreihe verwendet man den mit der wiederkehrenden Zahlung zu multiplizierenden Rentenbarwertfaktor. ${ }^{320}$ In der hier verwendeten Formelschreibweise symbolisiert wn die wirtschaftliche Nutzungsdauer (50 Jahre), während $n$ die Länge des Detailplanungshorizonts beschreibt (zehn Jahre):

$$
\frac{q^{w n-n}-1}{r \cdot q^{w n-n}}
$$

Man benötigt ferner den Kalkulationszinsfuß r, welcher jedoch aufgrund des Dilemmas der Lenkpreistheorie solange unbekannt ist, bis die optimale Lösung ermittelt wurde. Trotzdem wird für die Berechnung des Barwertfaktors schon bei Aufstellung des Modells ein Kalkulationszinsfuß benötigt: „Es bleibt nichts anderes übrig, als den internen Zinsfuß der günstigen Investition, die nicht mehr durchgeführt werden kann, zu schätzen." ${ }^{\text {"321 }}$

${ }^{317} \mathrm{Vgl}$. Abschnitt 2.2.4.

318 Vgl. JAENSCH (1966), S. 663, COENENBERG/SCHULTZE (2003), S. 122, PFNÜR (2011), S. 98, BALLWIESER/HACHMEISTER (2016), S. 66, TOLL/KINTZEL (2019), S. 1084. BUSSE VON COLBE (1957), S. 77 hält dies in vielen Fällen für eine sinnvolle Heuristik. Zu Herleitung und Berechnung der ewigen Rente vgl. HERING (2017), S. $46 \mathrm{f}$.

319 Vgl. WAMELING (2004), S. 69, HERING (2014), S. 40 f., BALLWIESER/HACHMEISTER (2016), S. 71.

${ }^{320}$ Dazu vgl. HAX (1985), S. 14 f., HERING/SCHNEIDER/TOLL (2009), S. 1115, HERING (2017), S. 46.

${ }^{321} H_{A X}$ (1964), S. 438. Er führt weiter aus: „Diese Lösung ist theoretisch vielleicht nicht restlos befriedigend. Man kann einerseits nicht mit unendlichen Planungszeiträumen arbeiten, muß also irgendwo 
Der Ansatz eines endlichen Barwerts trägt objektspezifischen Gegebenheiten in besonderem Maße Rechnung, weil die per Nutzwertanalyse maßgeschneiderten Zahlungsströme auch in die zweite Phase eingehen. Er berücksichtigt zudem, daß der Lebenszyklus von Eigentumswohnungen zwar ausgesprochen lang, aber nicht ewig währt und ein Verkauf in vielen Fällen nicht vorgesehen ist.

\subsubsection{Besonderheiten im Entscheidungsfeld einer Eigentumswohnung}

Das Entscheidungsfeld bei der Investition in Eigentumswohnungen ist von einigen besonderen Objekten und Rahmenbedingungen geprägt. Man findet diese vor allem im Bereich der Mittelherkunft und -bindung: Höchst selten wird der Kaufpreis allein aus eigenen Mitteln aufgebracht. ${ }^{322}$ In der Regel kann man die Wohnung nur deswegen kaufen, weil die Bank mit einem langfristigen Darlehen ein komplementäres Finanzierungsobjekt zur Verfügung stellt. ${ }^{323}$ Meist ist es Kreditvoraussetzung, die Wohnung per Grundpfandrecht an die Bank zu verpfänden. ${ }^{324}$ Wenn bei Verkauf der Wohnung eine vorzeitige Rückzahlung des Darlehens veranlaßt werden muß, steht der Bank häufig vertraglich eine Vorfälligkeitsentschädigung zu. ${ }^{325}$

Wegen der hohen Kapitalbindung und langen Lebensdauer von Eigentumswohnungen sind Investitionsobjekt und Entscheidungsfeld stark miteinander verwoben. Man spricht auch von einem Hebeleffekt, weil der Kreditbetrag das Jahreseinkommen des Investors oft um ein Vielfaches übersteigt. ${ }^{326}$ Daher erfolgt die Finanzierung häufig sehr langfristig. Die vollständige Tilgung erwartet man oft erst nach etwa 30 bis 40 Jahren. ${ }^{327}$ Die

im Zeitablauf eine Grenze setzen, über die die Planung nicht hinausreicht; andererseits muß man, wenn man Fehlentscheidungen vermeiden will, auch Einnahmen und Ausgaben berücksichtigen, die jenseits dieser Grenze liegen; dies ist notwendig, weil manche Investitionsprojekte und Finanzierungsmaßnahmen im Planungszeitraum beginnen, aber erst später enden. Die Schätzung eines geeigneten Kalkulationszinsfußes für die Zeit nach dem Planungszeitraum dürfte der einzige Ausweg aus diesem Dilemma sein.“

${ }^{322}$ Vgl. JOKL (2009), S. 63 f., MARKMANN/MARKMANN/ROTTKE (2017), S. 642, ROTTKE (2017c), S. 145.

${ }^{323}$ Die häufigste Finanzierungsform in Deutschland stellen grundpfandrechtlich besicherte Darlehen von Banken und Versicherungen dar, vgl. SCHÄFERS/HOLZMANN/SCHULTE/LANG/SCHOLZ (2016), S. 488, BRAUER (2019c), S. 437.

${ }^{324}$ Vgl. WESTERHEIDE (2017), S. 496. Über eine Grundschuld oder Hypothek wird die Immobilie an das Darlehen gekoppelt, dazu auch ROTTKE (2017e), S. 905 f., ROTTKE/EIBEL (2017), S. 852 f. Einen Überblick über Modalitäten grundpfandrechtlicher Besicherung gibt $\operatorname{HELLERFORTH}(2008)$, S. 63 ff., NOOSTEN (2015), S. 46 f. Zur grundpfandrechtlichen Belastung von Wohnungseigentum vgl. SCHNEIDER (2017), Rn. $175 \mathrm{ff}$.

${ }^{325}$ Diese soll den Schaden durch entgangene Zinsen bis zum Ende der Zinsbindungsfrist ersetzen. Zum Vorfälligkeitsentgelt vgl. PFNÜR (2011), S. 150, BRAUER (2019c), S. 429 f.

${ }^{326}$ Vgl. auch zum Folgenden HELLERFORTH (2012), S. 11, RENNERT (2012), S. 18, SCHÄFERS/ HOLZMANN/SCHULTE/LANG/SCHOLZ (2016), S. 486.

${ }^{327}$ Vgl. SCHULZE/STEIN/TIETGEN/MÖLLER (2017), S. 63, LINDMAYER/DIETZ (2018), S. 312 f., BRAUER (2019c), S. 426. 
Zinsbindung hingegen ist selten fristenkongruent. ${ }^{328}$ Es ist üblich, den Finanzierungszins nur für fünf, zehn oder fünfzehn Jahre festzuschreiben. ${ }^{329}$ Aus dem Zusammenspiel dieser Faktoren erwächst die besondere Relevanz der jederzeitigen Liquiditätswahrung: Wegen des erheblichen Hebeleffekts können bei Ablauf der zehnjährigen Zinsbindung auch bei vergleichsweise geringen Zinserhöhungen die monatlichen Kosten sprunghaft ansteigen und erhebliche Zahlungsschwierigkeiten hervorrufen. ${ }^{330}$ Ohnehin sind Eigentumswohnungen selten kurzfristig liquidierbar. ${ }^{331}$ Selbst bei finanziellen Engpässen dauert allein die Abwicklung der Transaktion eine gewisse Zeit, weil mit Bank, Grundbuchamt und Notar viele Akteure involviert sind. ${ }^{332}$

Bei Immobilienkrediten unterscheidet man hinsichtlich der Tilgungsmodalitäten zwischen Annuitäten-, Raten- und endfällig zu tilgenden Festdarlehen: ${ }^{333}$

- Annuitätendarlehen sind die in Deutschland verbreitetste Form der Immobilienfinanzierung. ${ }^{34}$ Die monatliche Rate, genannt Annuität, besteht aus Zins- und Tilgungsanteil. Während der gesamten Dauer der Zinsbindung bleibt sie konstant, durch fortwährende Tilgung nimmt die Restschuld im Laufe der Zeit sukzessive $\mathrm{ab}$ - und mit dieser auch die in der Rate enthaltene Zinslast, während der Tilgungsanteil steigt.

- Raten(tilgungs)darlehen weisen einen monatlich gleichbleibenden Tilgungsbetrag auf. ${ }^{335}$ Da die Restschuld sukzessive sinkt, nimmt auch die Zinslast immer weiter $\mathrm{ab}$ - und mit ihr die monatliche Rate.

- Festdarlehen oder endfällige Darlehen sind eine zweifelhafte Form der Immobilienfinanzierung. Während der gesamten Laufzeit ist keinerlei Tilgung vorgesehen, es werden ausschließlich Zinsen gezahlt. ${ }^{336}$ Ein sog. Tilgungsersatzmittel, z.B. eine Kapitallebensversicherung, soll erst am Laufzeitende zur vollständigen Rückzahlung der Schuld dienen. Die Vorteilhaftigkeit dieses „Wackelmodells“

\footnotetext{
${ }^{328}$ Vgl. HELLERFORTH (2008), S. 77, KELLER (2013a), S. 552 f., SCHULTE/SOTELO/ALLENDORF/ ROPETER-AHLERS/LANG (2016), S. 585 f. Zur historischen Entwicklung der sog. Abschnittsfinanzierung vgl. JOKL (2009), S. 63 f.

${ }^{329}$ Vgl. PFNÜR (2011), S. 149, RENNERT (2012), S. 121.

${ }^{330}$ Vgl. JOKL (2009), S. 67 f., BRUHN (2009), S. 211, ROTTKE (2017a), S. 96.

${ }^{331}$ Vgl. dazu ROTTKE (2017b), S. 121, MARKMANN/MARKMANN/ROTTKE (2017), S. 636.

${ }^{332}$ Zum Vorgang des Immobilienkaufs, insbesondere zur notariellen Beurkundung, Auflassung und Ablösung ehemaliger Darlehen vgl. STEINFORTH (2019), S. 79 ff.

${ }^{333}$ Vgl. HELLERFORTH (2012), S. 121 f., SCHÄFERS/HOLZMANN/SCHULTE/LANG/SCHOLZ (2016), S. $489 \mathrm{ff}$.

${ }^{334}$ Vgl. zu dieser Finanzierungsform RENNERT (2012), S. 119, ROTTKE/EIBEL (2017), S. 856, FESSELMANN/KOBABE/SCHWENZFEIER/SIEGEMUND (2018), S. 64, BRAUER (2019c), S. 426.

${ }^{335}$ Vgl. zur dieser Kreditart KELLER (2013a), S. 443, SCHULZE/STEIN/TIETGEN/MÖLLER (2017), S. 59 f.

${ }^{336} \mathrm{Zu}$ dieser Art der Finanzierung vgl. ROTTKE (2017e), S. 911 f., BRAUER (2019c), S. 427 f.
} 
wird durch die steuerliche Absetzbarkeit von Schuldzinsen im Rahmen von Vermietungseinkünften propagiert. ${ }^{337}$ Bis zum Jahr 2005 waren Habenzinsen aus Lebensversicherungen zudem steuerfrei und untermauerten die Argumentation. ${ }^{338}$

\subsubsection{Tabellarische Zusammenfassung bewertungsrelevanter Zahlungen}

Tabelle 9 faßt die Zahlungen zu den unterschiedlichen Zeitpunkten zusammen:

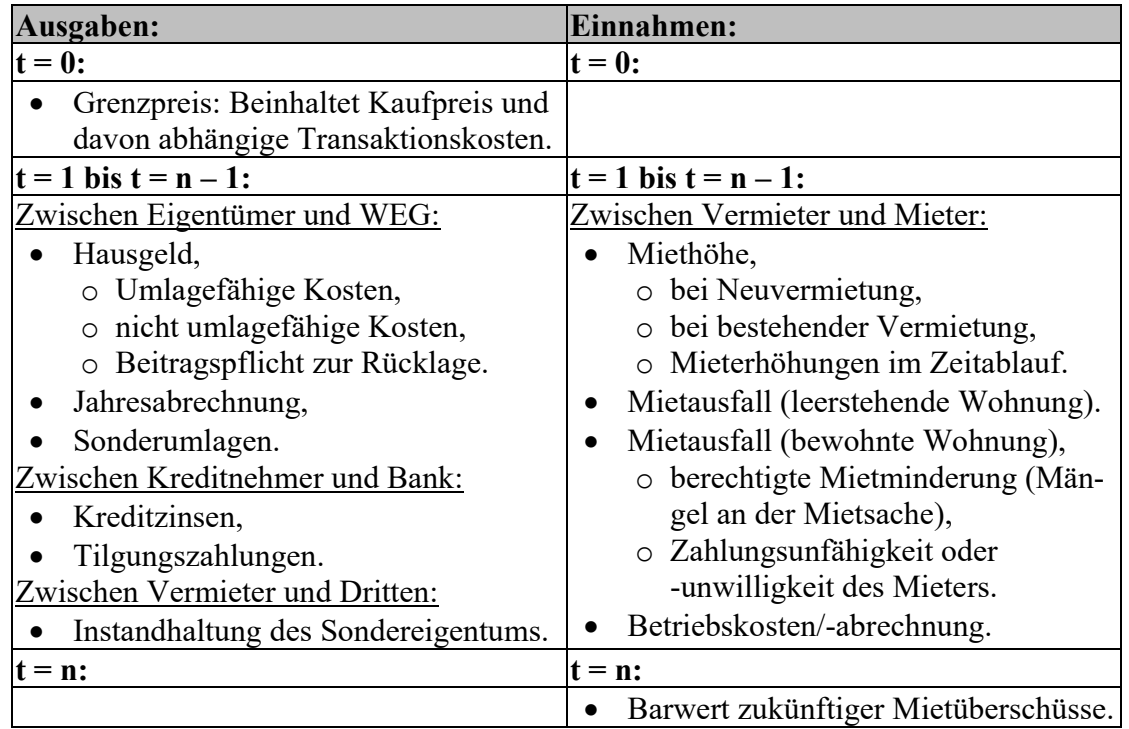

Tabelle 9: Zahlungsstrom-Komponenten einer vermieteten Eigentumswohnung

\subsubsection{Zustands-Grenzpreismodell als anwendbares Totalmodell}

\subsubsection{Grundlagen und Vorläufermodelle}

Ein zielführendes Totalmodell zur Bewertung von Eigentumswohnungen im Rahmen der Entscheidungsfunktion ist das Zustands-Grenzpreismodell (ZGPM). ${ }^{339}$ Es berück-

337 „Immobilienkäufer wurden leider durch unausgegorene Steuerargumente häufig verleitet, die eigentlich entscheidenden Faktoren für die Rentabilität der Immobilie aus dem Blick zu verlieren. [...] Der Immobilienkäufer muss sich darüber im Klaren sein, dass Verluste aus einer Immobilie nur teilweise durch Steuererstattungen kompensiert werden können und zwar maximal in Höhe des Spitzensteuersatzes des Immobilieneigentümers. Wenn Sie also einen Spitzensteuersatz von $40 \%$ haben, dann können Sie über Steuererstattungen auch nur maximal $40 \%$ von aufgelaufenen Verlusten aus der Vermietung kompensieren, aber niemals 100\% oder gar mehr." RENNERT (2012), S. 86 ff. Zur Kritik an endfälligen Darlehen vgl. auch BRÜBACH(2005), S. 74: „,Berater“ verkaufen ,Steuersparprodukte“ ohne Rücksicht auf Risiken".

${ }^{338}$ Vgl. LINDMAYER/DIETZ (2018), S. 323 ff.

${ }^{339}$ Zum ZGPM vgl. HERING (2000a). 
sichtigt per Definition neben dem Bewertungsobjekt auch die Zielsetzung und das Entscheidungsfeld des Bewertungssubjekts unter den realistischen Bedingungen des unvollkommenen Kapitalmarkts.

Dem ZGPM liegen die linearen Optimierungsansätze der Modelle von HAX und WEINGARTNER zugrunde. ${ }^{340}$ Es ist in der Lage, vielseitige Interdependenzen und Restriktionen flexibel zu erfassen und kann u.a. zur Bewertung der Zahlungsströme von Unternehmen, Immobilien und insbesondere Eigentumswohnungen eingesetzt werden. ${ }^{341}$ Das ZGPM kombiniert die Vorzüge der Vorläufermodelle von LAUX und FRANKE ${ }^{342}$ sowie JAENSCH und MATSCHKE, ${ }^{343}$ während es ihre Nachteile umgeht: ${ }^{344}$ Wie schon das Modell von JAENSCH und MATSCHKE ermittelt auch das ZGPM den Grenzpreis durch einen eleganten Vorher-Nachher-Vergleich zwischen Basis- und Bewertungsprogramm. Im Unterschied dazu verklebt es die modellierten Objekte jedoch nicht zu amalgamierten Erfolgsziffern, ${ }^{345}$ sondern modelliert den unvollkommenen Kapitalmarkt im Zeitablauf - so wie auch bereits das Modell von LAUX und FRANKE, ${ }^{346}$ welches jedoch den Grenzpreis in einem einzigen, aufwendigen Schritt ermittelt und den Preis so lange parametrisch variiert, bis der Kauf unvorteilhaft wird und das Bewertungsobjekt aus dem optimalen Programm ausscheidet.

Im ersten Schritt betrachtet das ZGPM den Ausgangsfall. Aus Sicht des Käufers ist dies die Situation ohne Bewertungsobjekt. Gesucht ist das optimale Investitions- und Finanzierungsprogramm, bestehend aus denjenigen bereits verfügbaren Objekten, die im $\mathrm{Zu}-$ sammenwirken den höchsten Entnahmestrom ermöglichen. ${ }^{347}$ Das Ergebnis nennt man Basisprogramm. Im zweiten Schritt werden die Rahmenbedingungen durch Zugang des Bewertungsobjekts modifiziert. Nun ermittelt das Modell das optimale Programm einschließlich Bewertungsobjekt. Dieses nennt man Bewertungsprogramm. Der Grenzpreis geht aus dem Vergleich der Vorteilhaftigkeit beider Programme hervor. Er beinhaltet neben dem Ertragswert des Bewertungsobjekts auch die Kapitalwertänderung aufgrund der Umstrukturierung bei Übergang vom Basis- zum Bewertungsprogramm. ${ }^{348}$

${ }^{340}$ Vgl. WEINGARTNER (1963), HAX (1964).

${ }^{341}$ Zur Anwendung des ZGPM im Rahmen der Immobilienbewertung vgl. KEUPER/PAPE (2008) (Wohnungsunternehmen), HARES (2011), S. 102 ff. (Immobilienverkauf), TOLL/WALOCHNIK (2013), S. $26 \mathrm{ff}$. (Wohnimmobilien).

${ }^{342} \mathrm{Vgl}$. LAUX/FRANKE (1969), S. $210 \mathrm{ff}$.

${ }^{343}$ Vgl. JAENSCH (1966), S. 664 f., MATSCHKE (1972), S. 155 ff., MATSCHKE (1975), S. 249 ff.

344 Auch zu Folgendem vgl. HERING (2014), S. 48.

${ }^{345}$ Zur „Amalgamationsfunktion“ vgl. MATSCHKE (1975), S. 105 ff.

${ }^{346} \mathrm{Vgl}$. LAUX/FRANKE (1969), S. 207.

${ }^{347}$ Vorliegend wird vom Fall der Einkommensmaximierung ausgegangen, vgl. dazu HERING (2014), S. 50 ff. Zur Vermögensmaximierung vgl. HERING (2000a), S. 364 ff., HERING (2014), S. 60 ff.

${ }^{348} \mathrm{Vgl}$. HERING/TOLL (2017b), S. $200 \mathrm{f}$. 


\subsubsection{Zweistufige Bewertung mit Hilfe linearer Optimierung}

\subsection{Ermittlung des optimalen Basisprogramms ohne Eigentumswohnung}

Das Aufstellen des Optimierungsansatzes beginnt damit, die Zielfunktion individuell auf die Konsumpräferenzen des Bewertungssubjekts zuzuschneiden: Je nach gewünschtem Entnahmemuster sieht sie entweder vor, GW als Summe aller Vermögensentnahmen $V_{t}$ zu den Zeitpunkten $t^{349}$ oder die Breite eines strukturierbaren Einkommensstroms EN zu maximieren. ${ }^{350}$ Durch Formulierung des Gewichtungsvektors w (bei Vermögensmaximierung) bzw. $\bar{w}$ (bei Einkommensmaximierung) ist gewährleistet, daß nahezu jede beliebige zeitliche Struktur von $V_{t}$ oder EN vom Modell erfaßt werden kann. Maßstab der zeitlichen Dimension sind die Perioden $t=(0,1, \ldots, n)$. Der Planungszeitraum erstreckt sich vom Entscheidungszeitpunkt $t=0$ bis zum Ende des Planungshorizonts, genannt $\mathrm{t}=\mathrm{n}$.

Das Entscheidungsfeld umfaßt Investitions- und Finanzierungsobjekte $j=(1, \ldots, m)$. Der Parameter $g_{j t}$ bezeichnet die Zahlungsüberschüsse des Objektes j zum Zeitpunkt t. Die Variable $x_{j}$ beschreibt seine Durchführungshäufigkeit. Falls ein Objekt nur bis zu einer bestimmten Höchstgrenze durchgeführt werden kann, wird dies durch die Obergrenzenrestriktion $x_{j}^{\max }$ gekennzeichnet. Feststehende Zahlungen zum Zeitpunkt $t$ werden durch $b_{t}$ ausgedrückt. Dies können z.B. Fixkosten sein, ein feststehendes Einkommen oder als sicher angenommene Mieteinnahmen aus anderweitiger Quelle. Wie bereits erwähnt, können Zahlungen, die jenseits des Planungshorizonts auftreten, als Barwert dargestellt werden, der mit einem pauschal geschätzten Zinssatz berechnet wird. ${ }^{351}$ Dies gilt nicht nur für den Zahlungsstrom des Bewertungsobjekts, sondern auch für alle anderen Investitions- und Finanzierungobjekte. ${ }^{352}$ Die Elemente w bzw. $\overline{\mathrm{w}}$ des Gewichtungsvektors sind am Planungshorizont ebenfalls analog zum Barwertfaktor zu gewichten. ${ }^{353}$

Relevante Zusammenhänge und Wechselwirkungen können (und müssen!) einzelfallabhängig erfaßt werden. ${ }^{35}$ Eine Abbildung des Entscheidungsfelds ist durch kreative

${ }^{349}$ Zur Ermittlung des Basisprogramms bei Vermögensmaximierung siehe HERING (2000a), S. 364.

${ }^{350}$ Zur Ermittlung des Basisprogramms bei Einkommensmaximierung siehe HERING (2014), S. 50 f. Die im Folgenden verwendeten Symbole orientieren sich ebd. - abgesehen vom Symbol für Vermögensentnahmen, wegen Symbolgleichheit mit der weiter unten definierten Kassenhaltung.

${ }^{351}$ Vgl. Gliederungspunkt 2.2.3.3.4.

${ }^{352}$ Wenn dies jedoch erforderlich ist, sollte man hinterfragen, ob die Modellierung bereits optimal ist, da bei der Abgrenzung des Planungshorizonts eine Orientierung am längsten verfügbaren Zahlungsstrom sinnvoll ist, vgl. HERING (2017), S. 14. Auch SCHNEIDER (1992), S. 29 schlägt vor, sich bei der Untergrenze des Planungshorizonts situativ an einzelnen Handlungsalternativen zu orientieren.

${ }^{353} \mathrm{Vgl}$. TOLL/HERING (2017), S. 475.

${ }^{354} \mathrm{Zu}$ den folgenden Nebenbedingungen vgl. HAX (1985), S. 86 ff., HERING (2014), S. 50. Zu weiteren Nebenbedingungen siehe $\operatorname{HAX}(1964)$, S. 442 f. Zur Modellierung vielfältiger Nebenbedingungen im Rahmen linearer Optimierung vgl. auch SUHL/MELLOULI (2013), S. $103 \mathrm{ff}$. 
Modellierung von Nebenbedingungen auf vielfältige Weise möglich, welche sich auf Handlungsmöglichkeiten, Interdependenzen und Restriktionen beziehen. Das Modell ist ausgesprochen flexibel, weil es die Anpassung an subjektive Gegebenheiten erlaubt, anstatt dem Bewertungssubjekt unumstößliche Rahmenbedingungen aufzuzwingen. ${ }^{355}$ An erster Stelle ist die Zahlungsfähigkeit zu nennen, die zu jedem Zeitpunkt gewährleistet sein muß. Liquiditätsnebenbedingungen fordern, daß Mittelzuflüsse aus Investitionsobjekten und exogenen Zahlungen in jedem Zeitpunkt ausreichen, um Entnahmen zu gewährleisten und Zahlungsverpflichtungen nachzukommen, z.B. aufgrund von Finanzierungsobjekten, Gehaltszahlungen oder Mietverträgen. Es muß im Einzelfall überlegt werden, an welcher Stelle Nichtnegativitätsbedingungen vorzugeben sind, beispielsweise für die Durchführungshäufigkeiten realwirtschaftlicher Investitionen. Auch die Entnahmegröße EN muß größer null sein, damit das Basisprogramm vorteilhaft ist. Die Abbildung unteilbarer Objekte ist durch Formulierung von Ganzzahligkeitsbedingungen möglich. Weitere gängige Nebenbedingungen sind Synergie- oder Diversifikationseffekte, die nur bei gemeinsamer Realisation bestimmter Objekte auftreten; sie können mathematisch ebenso erfaßt werden wie der gegenseitige Ausschluß von Objekten. ${ }^{356}$ Die Zielfunktion ist unter Beachtung der Nebenbedingungen zu maximieren.

Für den Fall der Einkommensmaximierung ergibt sich folgender Basisansatz: ${ }^{357}$ max. Entn; Entn $:=$ EN

$$
\begin{array}{ll}
-\sum_{j=1}^{m} g_{j 0} \cdot x_{j} \leq b_{0} & \\
-\sum_{j=1}^{m} g_{j t} \cdot x_{j}+\bar{w}_{t} \cdot E N \leq b_{t} & \forall t \in\{1,2, \ldots, n\} \\
x_{j} \leq x_{j}^{\max } & \forall j \in\{1,2, \ldots, m\} 358 \\
x_{j} \geq 0 & \forall j \in\{1,2, \ldots, m\} \\
E N \geq 0 &
\end{array}
$$

Der Ansatz kann mit Hilfe des Simplexalgorithmus gelöst werden. ${ }^{359}$ Als Ergebnis erhält man die Breite des Entnahmestroms als optimalen Zielfunktionswert und gleichzeitig die Durchführungshäufigkeiten aller Objekte des optimalen Basisprogramms.

\footnotetext{
${ }^{355} \mathrm{Zu}$ diesem Schluß kommen auch KEUPER/PAPE (2010), S. $73 \mathrm{f}$.

356 Vgl. dazu HAX (1985), S. 95 ff.

${ }^{357}$ Vgl. HERING (2014), S. 50 f.

358 „Diese Bedingung entfällt für alle $\mathrm{x}_{\mathrm{j}}$ mit $\mathrm{x}_{\mathrm{j}}^{\max }=\infty$.“ HERING (2014), S. 50.

${ }^{359}$ Zum Simplexalgorithmus vgl. DANTZIG (1966), S. 118 ff., MÜLLER-MERBACH (1973), S. 100 ff., DINKELBACH (1992), S. 11 ff., SUHL/MELLOULI (2013), S. 44 ff., WERNERS (2013), S. 44 ff., DOMSCHKE/DREXL/KLEIN/SCHOLL (2015), S. 26 ff.
} 


\subsection{Ermittlung des optimalen Bewertungsprogramms einschließlich}

Eigentumswohnung

Im zweiten Schritt erfolgt die Bestimmung des optimalen Bewertungsprogramms, aus welchem auch der gesuchte Grenzpreis hervorgeht. ${ }^{360}$ Der Optimierungsansatz wird um das Bewertungsobjekt U ergänzt, welches dem Programm zum Preis p hinzugefügt wird. Die feststehenden Zahlungsüberschüsse $b_{t}$ werden um die Einzahlungsüberschüsse aus dem Bewertungsobjekt ergänzt, genannt $\mathrm{g}_{\mathrm{Kt}}$. Durch Änderung der Zielfunktion ist nun der Grenzpreis p die zu maximierende Zielgröße. Hinzu tritt die Nebenbedingung, daß der bereits im Basisprogramm ermittelte optimale Zielfunktionswert EN* nicht unterschritten werden darf. Der Bewertungsansatz für den Fall der Einkommensmaximierung ist im Folgenden zusammengefaßt. Von hier an werden die Änderungen im Vergleich zum vorherigen Ansatz zur besseren Erkennbarkeit in blauer Schrift hervorgehoben:

$$
\begin{array}{ll}
\max . U ; U:=p & \\
-\sum_{j=1}^{m} g_{j 0} \cdot x_{j}+p \leq b_{0} & \\
-\sum_{j=1}^{m} g_{j t} \cdot x_{j}+\bar{w}_{t} \cdot E N \leq b_{t}+g_{K t} & \forall t \in\{1,2, \ldots, n\} \\
-E N \leq-E N^{*} & \\
x_{j} \leq x_{j}^{\max } & \forall j \in\{1,2, \ldots, \mathrm{m}\} \\
x_{j} \geq 0 & \forall j \in\{1,2, \ldots, m\} \\
E N \geq 0 & \\
p \geq 0 &
\end{array}
$$

Mit der Lösung des Optimierungsansatzes erhält man den gesuchten Grenzpreis $p^{*}$ und zusätzlich die im optimalen Bewertungsprogramm vorkommenden Objekte. Der Grenzpreis verkörpert den maximalen Kaufpreis der Wohnung einschließlich Erwerbsnebenkosten, bei dem die Transaktion wirtschaftlich gerade noch nicht nachteilig wird.

\subsection{Exemplarische Veranschaulichung des Bewertungsmodells}

\subsubsection{Zielsystem als Bewertungsausgangspunkt}

Nach der allgemeinen Darstellung soll die Bewertung einer Eigentumswohnung nun auch exemplarisch vorgeführt werden: Betrachtet sei ein Investor, der über den Kauf

${ }^{360}$ Zur folgenden Ermittlung des Bewertungsprogramms bei Einkommensmaximierung vgl. HERING (2014), S. 51 f. Zum Fall von Vermögensmaximierung vgl. HERING (2000a), S. 364 f. 
einer Eigentumswohnung zu Vermietungszwecken nachdenkt. Er habe bereits mehrere Objekte besichtigt und nach einer ersten Vorselektion eine Wohnung gefunden, die sich aus seiner Sicht als potentielles Bewertungsobjekt eignet. Um für den Verhandlungsprozeß gewappnet zu sein, muß er nun seinen Grenzpreis ermitteln. Für die Bewertung sind viele quantitative Eingangsdaten erforderlich.

Zuerst verschafft er sich Klarheit über seinen Planungshorizont: Einerseits weiß der Investor, daß die Qualität jedweder Prognose um so schlechter wird, je weiter sie in die Zukunft reicht. ${ }^{361}$ Andererseits ist ihm bewußt, daß er der ausgesprochenen Langlebigkeit des Wirtschaftsguts Immobilie nur durch einen entsprechend langen Planungshorizont gerecht werden kann. ${ }^{362}$ Wie bereits in Gliederungspunkt 2.2.3.3.4 vorweggenommen wurde, unterteilt er den Planungszeitraum in zwei Phasen: Innerhalb einer zehnjährigen Detailplanungsphase soll eine detaillierte Modellierung erfolgen. Angelehnt an die Abschreibungstabellen des Finanzamts schließt sich eine weitere, 40-jährige Phase an, in der nur der Zahlungsstrom des Bewertungsobjekts als Barwert abgebildet wird.

Den gedanklichen Grundstein zur Suche nach einer vermietbaren Eigentumswohnung legte sein Wunsch nach Erzielung eines möglichst langfristigen und gleichmäßigen Zusatzeinkommens. Diese Konsumpräferenz entspricht dem Streben nach Einkommensmaximierung und verlangt nach einer gleichmäßigen Gewichtung des Entnahmevektors über alle Perioden. Daher mißt der Investor den wiederkehrenden Entnahmen der ersten zehn Perioden das gleiche Gewicht bei. ${ }^{363}$ Zwar verfügen auch die Entnahmen der folgenden vierzig Jahre jenseits des Planungshorizonts gedanklich über die gleichen Gewichtungen; deren modellmäßige Abbildung erfolgt jedoch durch Zuweisung eines einzigen Gewichts in Form des Rentenbarwertfaktors am Ende des Planungshorizonts. Für die Berechnung des Barwertfaktors ist ein Kalkulationszinsfuß nötig, den man schätzen muß. Hierzu sollte man potentielle Grenzobjekte aus dem Entscheidungsfeld heranziehen. Es soll hier bereits vorweggenommen werden, daß aus heutiger Sicht vor allem die einperiodige Geldanlage (null Prozent) oder Kreditaufnahme (ein Prozent) in Frage kommen. ${ }^{364}$ Für den Rentenbarwert ist jedoch ein Zinssatz relevant, der in $\mathrm{t}=10$ gültig ist. Bis dahin hält der Investor einen Anstieg des Zinsniveaus zumindest für möglich. Dem Gedanken kaufmännischer Vorsicht folgend bevorzugt er die Verwendung eines möglichst hohen Zinssatzes, weil dieser zu einem tendenziell niedrigen Barwert führt. Er entscheidet sich daher für die Anwendung eines Zinssatzes von zwei Prozent, aus dem folgender Rentenbarwertfaktor hervorgeht:

$$
\frac{\mathrm{q}^{\mathrm{wn}-\mathrm{n}}-1}{\mathrm{r} \cdot \mathrm{q}^{\mathrm{wn}-\mathrm{n}}}=\frac{1,02^{50-10}-1}{0,02 \cdot 1,02^{50-10}}=\frac{1,02^{40}-1}{0,02 \cdot 1,02^{40}}=27,36
$$

\footnotetext{
${ }^{361}$ Vgl. BALLWIESER (1990), S. 26, ADAM (1996), S. 42, MANDL/RABEL (1997), S. 211.

${ }^{362} \mathrm{Vgl}$. SCHULTE/SOTELO/ALLENDORF/ROPETER-AHLERS/LANG (2016), S. 583, LEOPOLDSBERGER/ THOMAS/NAUBEREIT (2016), S. $448 \mathrm{f}$.

${ }^{363}$ Auch zu Folgendem vgl. HERING (2014), S. 50, TOLL/HERING (2017), S. 475.

${ }^{364}$ Auf diese Zinssätze wird in Abschnitt 2.3.3 noch genauer eingegangen.
} 
Der Faktor repräsentiert alle Entnahmen zwischen der elften und der fünfzigsten Periode, wird aber bereits in der zehnten Periode als zusätzliche Entnahme angesetzt - und daher zum Gewichtungsfaktor der zehnten Periode addiert. ${ }^{365}$ Somit kann $\overline{\mathrm{w}}_{10}=1+$ $\frac{1,02^{40}-1}{0,02 \cdot 1,02^{40}}=28,36$ als Element des Entnahmevektors in $\mathrm{t}=10$ angewendet werden. Dies führt zu einer Struktur des Entnahmevektors in Form von $\overline{\mathrm{W}}=(0 ; 1 ; 1 ; 1 ; 1 ; 1 ; 1$; $1 ; 1 ; 1 ; 28,36)$.

\subsubsection{Eigentumswohnung als Bewertungsobjekt}

\subsubsection{Beschreibung der Eigentumswohnung}

Potentielles Investitionsobjekt ist eine Eigentumswohnung im zweiten Obergeschoß eines Mehrfamilienhauses mit neun Wohnungen im nordrhein-westfälischen Viersen. Die 72,5 qm große Dreizimmerwohnung wird vom Eigentümer provisionsfrei zum Verkauf angeboten und liegt in zentraler Lage, nahe der Fußgängerzone der Kleinstadt mit etwa 75.000 Einwohnern. Die Nord-Süd-Autobahn A61 führt in die Fahrtrichtungen Venlo und Koblenz und ist drei Kilometer bzw. fünf Autominuten entfernt. Die Auffahrt zur Autobahn A52 in ost-westliche Richtung nach Roermond bzw. Düsseldorf liegt fünf Kilometer bzw. sieben Minuten entfernt.

Das Gebäude wurde 1968 errichtet, es gibt es keinen auffälligen Sanierungsstau. Von der Straßenseite gesehen bietet es keinen besonderen Glanz. Die vielen Bäume entlang der Straße wirken diesem unauffälligen Eindruck positiv entgegen. Die Parkplatzsituation ist schlecht, aber auf dem Grundstücksteil hinter dem Gebäude befindet sich der Garten, der links über eine große Wiese und rechts über je eine Garage pro Wohneinheit verfügt. ${ }^{366}$ Eine Besonderheit ist, daß es sich um zwei miteinander verbundene Reihenhäuser handelt, der Durchgang zwischen den beiden Treppenhäusern ist über den Kellerflur möglich. Im Gebäude befindet sich außerdem eine Arztpraxis. Der ständige Kundenverkehr im Treppenhaus steigert zwar den Reinigungsbedarf, kann aber hinsichtlich Einbruchsschutz als positiv gewertet werden, weil die starke Frequentierung viele Beobachter mit sich bringt und Einbrecher abschreckt, während Treppenhäuser von reinen Wohngebäuden die meiste Zeit des Tages verwaist sind. Die Innenausstattung der seit kurzem leerstehenden Wohnung ist zwar nicht modern, aber gut erhalten und zumindest nicht abschreckend. Sie besteht aus Innentüren und Fenstern mit braunen Holzrahmen, hellbraunem Parkett sowie Rauhfasertapete. Heutzutage verwendet man häufig großflächige Fliesen anstatt Holzböden und weiße Türen mit Beschlägen aus Edelstahl. Die

\footnotetext{
365 Vgl. TOLL/HERING (2017), S. 475.

${ }^{366}$ Der Garten einer WEG ist Gemeinschaftseigentum, weil er nicht räumlich abgeschlossen und damit nicht sondereigentumsfähig ist, vgl. SCHNEIDER (2017), Rn. 51 ff. Grundsätzlich kann die Teilungserklärung jedoch ein Sondernutzungsrecht am Garten bzw. dessen Teilflächen zugunsten bestimmter Eigentümer begründen, vgl. MÜLLER (2015b), Rn. 8. Wenn dies nicht geschieht, werden Gemeinschaftsgärten in der Praxis selten von jemandem genutzt und dienen nur der - hier durchaus schönen - Aussicht. Einen Überblick zu den Rechten und Pflichten der Eigentümer am Gemeinschaftsgarten gibt SUILMANN (2018c), Rn. 39 f.
} 
Wohnung könnte renoviert werden, weil sie modische Anforderungen nicht erfüllt. ${ }^{367}$ Dies ist jedoch Geschmackssache. Es besteht zumindest kein zwangsläufiger Renovierungsbedarf. ${ }^{368}$ Präferenzen und Bedürfnisse möglicher Mieter sind unterschiedlich und es ist wichtig, sich nach diesen zu richten. ${ }^{369}$ Die Wohnung erscheint dem Investor für die ländliche Gegend geeignet und vermarktungsfähig, zumal das Badezimmer in den 90er Jahren in weißer Farbe renoviert wurde. Es gibt also keine farbig abschreckenden Fliesen. Erfreulicherweise ist ein Balkon mit Markise vorhanden, von dem man Vögel und Bäume auf der Wiese hinter dem Gebäude beobachten kann.

Ältere Gebäude müssen keine schlechten Investitionsobjekte sein. Ganz im Gegenteil:370 Insbesondere bei Neubauten drohen ausufernde Wartungskosten, wenn umfangreiche technische Ausstattung vorhanden ist. Dem Verfasser ist aus seiner Tätigkeit als WEGVerwalter ein Neubau-Wohngebäude mit Tiefgarage bekannt. Letztere verfügt u.a. über vielfältige Brandschutztechnik und zieht enorme Kosten nach sich. ${ }^{371}$ Sind zudem Aufzüge vorhanden, finden quartalsweise Wartungen und jährliche TÜV-Prüfungen statt. ${ }^{372}$

${ }^{367}$ Zur Wohnwertverbesserung durch Renovierung und Modernisierung vgl. SCHULZE/STEIN/TIETGEN/ MÖLLER (2017), S. 84, KRÜGER/ROSDÜCHER (2017), S. 574.

${ }^{368}$ Zwar warnen SCHULZE/STEIN/TIETGEN/MÖLLER vor der schlechteren Vermarktungsfähigkeit älterer Bausubstanz, weisen jedoch darauf hin, daß auch energetische Sanierungen oft zuviel des Guten sind und die Vermietbarkeit sogar verschlechtern können, vgl. SCHULZE/STEIN/TIETGEN/MÖLLER (2017), S. 122 f., vgl. ähnlich auch NIERMANN/SCHNUR/DRILLING (2014), S. 19, dort in Bezug auf Mieterhöhungen nach § 559 BGB im Nachgang energetischer Investitionen und ihrem Konfliktpotential, weil sie - entgegen der Idealvorstellung - nicht immer korrespondierende Nebenkostenersparnisse mit sich bringen.

${ }^{369} \mathrm{Zu}$ Vermietbarkeit, Mieterpräferenzen, subjektivem Geschmack und Emotionalität vgl. WURZEL (2018), S. 10, BRAMMER/DZIOMBA (2018), S. 999 f. Zur starken Abhängig der Zielgruppe von der Mikrolage vgl. SCHARMANSKI/WIENCKE (2017), S. $732 \mathrm{ff}$. Zur Duldungspflicht des Vermieters bei der Gestaltung der Wohnung durch den Mieter (und deren Grenzen) vgl. HÄUBLEIN (2016a), Rn. 75.

${ }^{370}$ Zum Vergleich „Neubau oder Gebrauchtobjekt“ vgl. SCHULZE/STEIN/TIETGEN/MÖLLER (2017), S. $16 \mathrm{ff}$.

${ }^{371}$ In der Tiefgarage des Gebäudes befinden sich 11 Doppel- und 16 Einzelstellplätze. Die Nebenkosten (nicht Miete!) pro Einzelstellplatz lagen für das Gesamtjahr 2018 bei etwa 500 EUR, bei Doppelstellplätzen waren es ca. 840 EUR. Grund für die extremen Kosten ist - neben gewöhnlichen Grundbesitzabgaben, Verwaltungs- und Hausmeisterkosten - vor allem umfangreiche Gebäudetechnik. Insgesamt entfielen 2018 auf die gesamte Tiefgarage etwa 4.100 EUR Beleuchtungskosten, 4.300 EUR planmäßige Wartungskosten für die Sprinkleranlage zzgl. 400 EUR für deren TÜV-Prüfung, 1.000 EUR für planmäßige Wartung der Tiefgaragenabluftanlage, 200 EUR planmäßige Wartung des Tiefgaragentors sowie 200 EUR Kosten der TÜV-Prüfung von Notbeleuchtung und ihrer Stromversorgung, in Summe 10.200 EUR für 38 Stellplätze. Auf Tiefgarage und Wohngebäude gemeinsam entfielen weitere Kosten: Etwa 700 EUR planmäßige Wartungskosten für die Brandmeldeanlage sowie 3.300 EUR für deren Aufschaltung zur Feuerwehr sowie 800 EUR planmäßige Wartungskosten der Brandschutzklappen, in Summe nochmals 4.800 EUR. Diese laufenden Wartungskosten beinhalten keinerlei Reparaturen und keinerlei außerplanmäßige Kosten.

${ }^{372} \mathrm{Zu}$ Aufzugskosten vgl. LANGE (2018), S. 566, zu Betreibervorschriften vgl. FRITSCH (2018), Rn. 814 ff. Im vorgenannten Mehrfamilien-Doppelhaus befinden sich zwei Aufzüge. Die Kosten eines Aufzugs lagen im Gesamtjahr 2018 bei ca. 3.800 EUR für planmäßige Wartung zzgl. 350 EUR für die Zwischenprüfung durch den TÜV sowie 550 EUR Stromkosten. Das vorgeschriebene, von 
Das Viersener Gebäude hingegen verfügt - abgesehen von Heizung und Dach - über keinerlei Anlagen mit Wartungsaufwand. ${ }^{373}$ Angelehnt an Gliederungspunkt 2.2.3.2.3 nimmt der Investor eine Einordnung der Wohnung anhand dort genannter Merkmale vor. Diese sind in Tabelle 10 zusammenfassend dargestellt:

\begin{tabular}{|l|l|}
\hline Merkmal & Merkmalsausprägungen \\
\hline Gesamte Immobilie & \multicolumn{2}{|l|}{} \\
\hline Lage & $\bullet \quad$ Fußläufige Nähe zur Fußgängerzone. \\
& $\bullet \quad$ Kreisangehörige Stadt Viersen, 75.000 Einwohner. \\
\hline Gebäudedaten & $\bullet \quad$ Bestandsgebäude, Baujahr 1968. \\
& $\bullet \quad$ Neun Wohn- bzw. Teileigentumseinheiten. \\
\hline Sondereigentum & $\bullet \quad 691,25$ qm Gesamtwohnfläche. \\
\hline Wohnungsdaten & $\bullet \quad 2$. OG links. \\
& $\bullet \quad 72,56$ qm Wohnfläche. \\
\hline Nutzungsart & $\bullet \quad$ Wohneinheit einschließlich Garage. \\
\hline Sanierungsbedarf & $\bullet \quad$ Nicht modern, aber nicht zwingend renovierungsbedürftig. \\
\hline Derzeitige Nutzung & $\bullet \quad$ Leerstehend, grundsätzlich vermietbar. \\
\hline Gemeinschaftseigentum \\
\hline Nutzungsart & $\bullet \quad$ Gemischt genutztes Gebäude (Arztpraxis im EG). \\
\hline Sanierungsbedarf, & $\bullet \quad$ Alterstypisch durchschnittlicher Instandhaltungsbedarf. \\
\hline Wartungsintensität & $\bullet \quad$ Außer Dach und Zentralheizung keinerlei Wartungsaufwand. \\
\hline Besonderheiten & $\bullet \quad$ Viele Bäume im Garten. \\
\hline
\end{tabular}

Tabelle 10: Merkmale der exemplarischen Eigentumswohnung

\subsubsection{Prognose des Zahlungsstroms der Eigentumswohnung}

\subsection{Zahlungen im Investitionszeitpunkt}

Wie bereits in Unterabschnitt 2.2.3.3 erfolgt auch hier eine Einordnung nach der Zeit: Im Entscheidungszeitpunkt $\mathrm{t}=0$ findet lediglich die Zahlung des Kaufpreises statt, die nicht Eingangsdatum, sondern Ergebnis der Rechnung ist. Die Wohnung wird provisionsfrei durch den Eigentümer verkauft, daher ist nicht mit Maklercourtage zu rechnen, sondern nur mit Grunderwerbsteuer, Notar- und Grundbuchkosten. Diese summieren

beiden Aufzügen genutzte Notrufsystem rief Telefonanschlußkosten von 450 EUR zzgl. 1.200 EUR für die Aufschaltung zur Notrufzentrale hervor.

373 Ausufernde Gebäudetechnik kann ein Gebäude aufgrund hoher Folgekosten unattraktiv machen, vgl. LANGE (2019), S. 503. Zu Erst- und Folgekosten im Spannungsfeld zwischen vermiedener und ausufernder technischer Gebäudeausstattung bei Neubauten vgl. KURZROCK (2017a), S. 439. 
sich insgesamt auf etwa acht Prozent des Kaufpreises. ${ }^{374}$ Entsprechend ist der Entscheidungswert um den Faktor 1,08 zu bereinigen.

\subsection{Zahlungen während der Investitionsdauer}

Zuerst sammelt der Investor ortsübliche Durchschnittsdaten, bevor er im folgenden Schritt eine Individualisierung mit Hilfe einer Nutzwertanalyse vornimmt. Zu den wichtigsten Erfolgsdeterminanten zählen die laufenden Mieteinnahmen. Ausgangspunkt von deren Prognose ist die durchschnittliche Kaltmiete pro Quadratmeter. Obwohl ein Mietspiegel für die Stadt Viersen verfügbar ist, erscheinen dessen Daten dem Investor zu undifferenziert und zu alt. ${ }^{375}$ Er hält es für zielsetzungsgerechter, im Netz auf den gängigen Portalseiten nach Inseraten von mehr oder weniger vergleichbaren Objekte zu suchen. ${ }^{376}$ Damit die Suchergebnisse der Eigentumswohnung in möglichst vielen Merkmalen ähnlich sind, verwendet er die in Tabelle 11 abgebildeten Kriterien.

\begin{tabular}{|l|l|}
\hline Suchparameter: & Merkmalsausprägung: \\
\hline Baujahr bis: & 1997 ( $\geq$ 20 Jahre alt) \\
\hline Etage bis: & 4 \\
\hline Zimmer von: & 3 \\
\hline Zimmer bis: & 3 \\
\hline Quadratmeter von: & 65 \\
\hline Quadratmeter bis: & 75 \\
\hline Ort: & Viersen (Stadt) \\
\hline
\end{tabular}

Tabelle 11: Suchparameter für vergleichbare Eigentumswohnungen

Es erscheinen acht Suchergebnisse. Für den Investor erscheint die vergleichsweise geringe Trefferzahl nachrangig, Nähe und Ähnlichkeit zum Bewertungsobjekt sind ihm wichtiger. Deren Mittelwert hinsichtlich Kaltmiete pro Quadratmeter beträgt 5,92 EUR. Diesen Mietpreis rechnet er auf 72,56 qm und 12 Monate hoch und prognostiziert jährliche (Kalt-) Mieteinnahmen von 5.155,91 EUR.

Den auf den Mieter umlagefähigen Anteil des Hausgelds, also Heiz- und Betriebskosten, entnimmt er den vergangenen Jahresabrechnungen und bildet deren arithmetisches Mittel. Es ergibt sich ein Jahreswert von 1.830,07 EUR. Es wird unterstellt, daß diese Kosten vollständig an den Mieter weitergegeben werden können. Den nicht umlagefähigen Teil des Hausgelds schätzt der präsumtive Käufer, wobei er eine Differenzierung nach Instandhaltungs- und übrigen Kosten vornimmt: ${ }^{377}$ Für die jährlichen Instandhaltungskosten setzt er den Durchschnittswert aus der II. Berechnungsverordnung an. Er beträgt 11,50 EUR pro Quadratmeter und Jahr. ${ }^{378}$ Multipliziert mit der Fläche der Wohneinheit

\footnotetext{
${ }^{374} \mathrm{Vgl}$. Gliederungspunkt 2.2.3.3.1.

${ }^{375}$ Siehe Gliederungspunkt 2.2.3.3.3.1.

${ }^{376}$ Deutschlandweite Portale für Vermietung und Verkauf von Wohnungen und Häusern sind z.B. Immobilienscout, Immowelt und Immonet, vgl. dazu BRAUER (2019a), S. 13.

${ }^{377} \mathrm{Zu}$ diesen Kosten vgl. Gliederungspunkt 2.2.3.3.2.2.

${ }^{378}$ Vgl. § 28 Abs. 2 Nr. 3 II. BV, vgl. auch Gliederungspunkt 2.2.3.3.2.2.
} 
ergibt dies Instandhaltungskosten von 834,44 EUR p.a. Die übrigen nicht umlagefähigen Kosten übernimmt er aus Durchschnittswerten vergangener Jahresabrechnungen. Auf die Wohneinheit entfiel ein jährlicher Mittelwert von 363,52 EUR. Der Bezugsbasis einer jeden Position gebührt größte Aufmerksamkeit, damit sich bei der Hochrechnung keine Fehler einschleichen: Die Kaltmiete bezieht sich auf Quadratmeter und Monat, die Instandhaltungskosten auf Quadratmeter und Jahr, sonstige nicht umlagefähige Kosten beziehen sich auf Wohnung und Jahr. Als Eingangsdaten für die jährlichen Zahlungsströme werden jährliche Gesamtwerte benötigt. Es ergibt sich folgende Prognose:

Mietertrag:

$$
5,92 \mathrm{EUR} \cdot 72,56 \mathrm{qm} \cdot 12 \text { Monate } \quad=5.155,91 \mathrm{EUR}
$$

Umlagefähige Betriebskosten: ${ }^{379}$

$$
-1.830,07 \text { EUR } \quad \cdot \frac{0}{12} \text { Monate } \quad=0,00 \mathrm{EUR}
$$

Nicht umlagefähige Nebenkosten (ohne Instandhaltung):

$$
-363,52 \text { EUR } \quad=-363,52 \mathrm{EUR}
$$

Nicht umlagefähige Nebenkosten (nur Instandhaltung):

$$
-11,50 \text { EUR }
$$

Zahlungsstrom:

$$
\cdot 72,56 \mathrm{qm}
$$

$\equiv-834,44 \mathrm{EUR}$

\subsection{Nutzwertanalyse zur Anpassung von Durchschnittswerten an den Einzelfall}

Dem Investor ist bewußt, daß diese Werte viel zu pauschal sind. Er hält ihre Anpassung mit Hilfe einer Nutzwertanalyse für erforderlich, um objektindividuellen Gegebenheiten Rechnung zu tragen und diese in die Bewertung einfließen zu lassen. Dazu erstellt er eine Liste von Kriterien, welche die Zahlungsströme seiner Meinung nach beeinflussen können und gruppiert sie in folgende sechs, möglichst homogene Kategorien: ${ }^{380}$

- Wohnungseigentümergemeinschaft und Verwaltung,

- Bauqualität,

- Zustand und Erneuerungsbedarf,

- Ausstattung,

- Standortfaktoren unmittelbare Umgebung sowie

- Standortfaktoren Großraum.

Von den Kategorien erwartet der Investor in unterschiedlichem Maße Einfluß auf die beiden Rechtssphären Mietvertrag und Wohnungseigentümergemeinschaft, weshalb sich die Berechnung von zwei Nutzwerten anbietet. Jede Kategorie wirkt sich entweder

\footnotetext{
${ }^{379}$ Mögliche Leerstände werden vorerst ausgeblendet. Deren Berücksichtigung erfolgt im nächsten Gliederungspunkt durch Anwendung einer Nutzwertanalyse.

${ }^{380}$ Die vollständige Liste mit sämtlichen Kriterien befindet sich im Anhang 1.
} 
auf den Nutzwert MH (für Miethöhe), auf den Nutzwert NUNK (für nicht umlagefähige Nebenkosten) oder auf beide Nutzwerte aus. Sicherlich ist auf einem unvollkommenen Markt keine trennscharfe Zuordnung möglich, zumal zahlreiche Interdependenzen existieren. Dies ist dem Investor klar, letztlich ist die Auswirkung eines Kriteriums ebenso subjektiv wie auch deren Gewichtung. Er legt folgende Zuordnung fest:

- Die Kategorie Wohnungseigentümergemeinschaft und Verwaltung beeinflußt das Miteinander und somit die Fähigkeit aller Beteiligten, sich bei Problemen kooperativ zu verständigen, anstatt in endlosen Debatten oder Blockadehaltungen zu versinken. ${ }^{381}$ Durch diese Kategorie werden u.a. Hausgeldrückstände, Sanierungsstau und die Gefahr von kostenintensiven Rechtsstreitigkeiten innerhalb der WEG bewertet. Diese erste Kategorie ordnet er ausschließlich dem Nutzwert NUNK zu. ${ }^{382}$

- Die Kategorien Bauqualität sowie Zustand und Erneuerungsbedarf hält er für sowohl vermietungsrelevant als auch bedeutsam in Hinblick auf nicht umlagefähige Instandhaltungskosten. Das Erscheinungsbild des Gebäudes ist für den ersten Eindruck bei neuen Mietinteressenten bedeutsam. ${ }^{383}$ So kann man bei der Vermarktung der Wohnung entweder mit gehobener Bauqualität oder niedrigen, vom Mieter zu tragenden Wartungskosten werben. Die Frage ist auch für den Vermieter von Interesse: Sanierungsstau kann Folgeschäden und somit Mietminderungen innerhalb des Sondereigentums verursachen und so zu einem finanziellen Schaden führen.

- Die Kategorien Ausstattung und Standortfaktoren unmittelbare Umgebung sowie Standortfaktoren Großraum besitzen aus Sicht des Investors ausschließlich einen Stellenwert bei der Vermarktung gegenüber potentiellen Mietern. ${ }^{384}$ Wenn das Umfeld ansprechend, eine gute Verkehrsanbindung vorhanden und viele Freizeit- und Versorgungsmöglichkeiten in der Nähe verfügbar sind, ist die Nachfrage c.p. höher. Eine hochwertige Ausstattung des Gebäudes tut ihr Übriges.

Tabelle 12 zeigt die Zuordnung der Kategorien zu beiden Einflußbereichen. Die vollständigen Kriterien, deren Gewichtungen und Wertungen sind im Anhang $1 \mathrm{zu}$ finden, ebenso wie die schrittweise Ermittlung der Teilnutzwerte und die anschließende Berechnung beider Gesamtnutzwerte.

${ }^{381}$ Zur Relevanz einer guten Nachbarschaft und deren Auswirkungen auf Lebensqualität, Wohnzufriedenheit und Immobilienwert vgl. KEMPER/SCHÖFFEL (2014), S. 203.

${ }^{382}$ Eine komplizierte Wohnungseigentümergemeinschaft kann regelmäßig weitere Kosten verursachen, z.B. Sonderhonorare der Verwaltung oder Anwalts- und Gerichtskosten, vgl. dazu 2.2.3.3.3.2.

${ }^{383}$ Zum hohen Stellenwert des subjektiven Geschmacks bei Vermietung und Verkauf von Immobilien vgl. SCHULZE/STEIN/TIETGEN/MÖLLER (2017), S. 84, WURZEL (2018), S. 10.

${ }^{384}$ Auch zum Folgenden vgl. NIERMANN/SCHNUR/DRILLING (2014), S. 14, FELDMANN/GERSTNER/ HOFMANN/ISENHÄFER/SEGERER/VÄTH (2016), S. 392 f., SCHÜRT (2017), S. 75. 


\begin{tabular}{|l|l|}
\hline Kategorien der Nutzwertanalyse: & Relevanz? \\
\hline $\begin{array}{l}\text { Wohnungseigentümergemeinschaft und Verwaltung: } \\
\text { - Von Eigentümern abhängige Faktoren, }\end{array}$ & \\
- $\quad$ von Verwaltung abhängige Faktoren, \\
- $\quad$ gon Eigentümern und Verwaltung gemeinsam abhän- & Relevant für NUNK \\
- nicht/kaum beeinflußbare Faktoren. & \\
\hline $\begin{array}{l}\text { Bauqualität: } \\
\text { - Gesamtgebäude, }\end{array}$ & Relevant für MH und \\
- Wohneinheit. & NUNK \\
\hline $\begin{array}{l}\text { Zustand und Erneuerungsbedarf: } \\
\text { - Gesamtgebäude, }\end{array}$ & Relevant für MH und \\
- Wohneinheit. & NUNK \\
\hline Ausstattung: & Relevant für MH \\
- Gesamtgebäude und Grundstück, & \\
- Wohneinheit. & Relevant für MH \\
\hline Standortfaktoren unmittelbare Umgebung: & \\
\hline - Straße und unmittelbare Nachbarschaft, \\
- Infrastruktur, \\
- Versorgungs- und Freizeitmöglichkeiten.
\end{tabular}

Tabelle 12: Nutzwert-Kategorien

Die Berechnung ergibt die beiden Nutzwerte MH $(2,12)$ und NUNK $(2,16)$, die in den folgenden Tabellen wiedergegeben sind. ${ }^{385}$

\begin{tabular}{|l|r|r|r|r|}
\hline Die einzelnen Kategorien: & Relevant? & $\begin{array}{r}\text { Kategorie- } \\
\text { Gewicht }\end{array}$ & $\begin{array}{r}\text { Kategorie- } \\
\text { Nutzwert }\end{array}$ & Teilnutzwert \\
\hline WEG und Verwaltung & nein & - & - & - \\
\hline Bauqualität & ja & 63 & 1,9841 & 0,5682 \\
\hline Zustand und Erneuerungsbedarf & ja & 44 & 1,8182 & 0,3636 \\
\hline Ausstattung & ja & 56 & 2,0893 & 0,5318 \\
\hline $\begin{array}{l}\text { Standortfaktoren } \\
\text { unmittelbare Umgebung }\end{array}$ & ja & 38 & 2,3684 & 0,4091 \\
\hline Standortfaktoren Großraum & ja & 19 & 2,8947 & 0,2500 \\
\hline
\end{tabular}

Tabelle 13: Berechnung des Nutzwerts MH

${ }^{385}$ Der Rechenweg im Detail ist in Anhang 1 zu finden. 


\begin{tabular}{|l|r|r|r|r|}
\hline Die einzelnen Kategorien: & Relevant? & $\begin{array}{r}\text { Kategorie- } \\
\text { Gewicht }\end{array}$ & $\begin{array}{r}\text { Kategorie- } \\
\text { Nutzwert }\end{array}$ & Teilnutzwert \\
\hline WEG und Verwaltung & ja & 63 & 2,5714 & 0,9529 \\
\hline Bauqualität & ja & 63 & 1,9841 & 0,7353 \\
\hline Zustand und Erneuerungsbedarf & ja & 44 & 1,8182 & 0,4706 \\
\hline Ausstattung & nein & - & - & - \\
\hline $\begin{array}{l}\text { Standortfaktoren } \\
\text { unmittelbare Umgebung }\end{array}$ & nein & - & - & - \\
\hline Standortfaktoren Großraum & nein & - & - & - \\
\hline
\end{tabular}

\section{Tabelle 14: Berechnung des Nutzwerts NUNK}

Die gewonnenen Nutzwerte sind auf einen Wertebereich zwischen null und vier normiert. Zur situationsspezifischen Anpassung der durchschnittlichen Vergleichswerte an die vorliegende Immobilie werden sie in die gleichnamigen Faktoren MH und NUNK sowie in den Faktor MOL (für Monate ohne Leerstand) überführt, Tabelle 15 zeigt Rechenweg und Ergebnisse. ${ }^{386}$

\begin{tabular}{|l|r|r|r|}
\hline Kennzahl & \multicolumn{1}{|l|}{ Berechnung } & Ausprägung & Wertebereich \\
\hline MH & $\left(\mathrm{NW}_{\mathrm{MH}} \cdot 0,1+0,8\right) \cdot 100$ & $\mathbf{1 0 1 , 2 3 \%}$ & $80 \%-120 \%$ \\
\hline MOL & $\begin{array}{r}\left(\text { wenn } \mathrm{NW}_{\mathrm{MH}}<1 \mathrm{dann} \mathrm{NW}_{\mathrm{MH}} \cdot 10,5\right. \\
\left.\text { sonst } 10,5+\left(\mathrm{NW}_{\mathrm{MH}}-1\right) \cdot 0,5\right)\end{array}$ & $\mathbf{1 1 , 0 6} \mathbf{M o n}$. & $0-12 \mathrm{Mon}$. \\
\hline NUNK & $\left(2-\left[\mathrm{NW}_{\mathrm{NUNK}} \cdot 0,50\right]\right) \cdot 100$ & $\mathbf{9 2 , 0 6 \%}$ & $0 \%-200 \%$ \\
\hline
\end{tabular}

Tabelle 15: Berechnung von Kennziffern auf Nutzwertbasis

Mit diesen Kennzahlen können nun die auf Durchschnittswerten basierenden Zahlungsströme an das vorliegende Gebäude angepaßt werden. Zunächst wird die ortsübliche Kaltmiete mit dem Faktor MOL multipliziert. Dieser deutet auf jährlich ca. elf Monate ohne Leerstand hin. Anschließend wird die Miete mit dem Faktor MH multipliziert, der eine Miethöhe von rund 101 Prozent eines Vergleichs- oder Durchschnittswertes signalisiert und somit zu keiner wesentlichen Veränderung der durchschnittlichen Zahlungsströme führt. ${ }^{387}$ Es ergibt sich eine jährliche Kaltmiete von 4.810,95 EUR.

Durch den Faktor MOL verändert sich die Bedeutung umlagefähiger Nebenkosten. Der Investor geht nun von jährlich knapp einem Monat Leerstand aus und muß den entsprechenden Teil der umlagefähigen Betriebskosten selbst tragen. Die aus dem Durchschnitt vergangener Jahresabrechnungen ermittelten Kosten von 1.830,07 EUR werden mit 0,94 Monaten Leerstand multipliziert und führen zu einer jährlichen Belastung in Höhe von 143,15 EUR. Der Wert der Instandhaltungskosten von 11,50 EUR aus der II. BV

${ }^{386}$ Vgl. auch Gliederungspunkt 2.2.3.3.3.3.

${ }^{387}$ Auch die Erkenntnis, daß man einen Umweg nicht benötigt hätte, kann das Ergebnis einer Analyse sein. Angesichts der Tragweite der Entscheidung darf der Investor trotzdem nicht einfach abwarten, welche Werte sich einstellen, und sich bis dahin mit ungenauen Durchschnittsdaten zufrieden geben. Aufgrund von Hebeleffekt und hoher Kapitalbindung ist eine sorgfältige Analyse erforderlich, um eine Vorstellung zu erlangen, inwieweit sich die Daten in die Zukunft hochrechnen lassen. Ähnlich, jedoch bzgl. zukünftiger Zahlungsströme im Allgemeinen, äußert sich HERING (2017), S. 402. 
ist höchst pauschal. ${ }^{388}$ Er wird mit dem Faktor NUNK angepaßt, der einen überdurchschnittlich guten Wert aufweist und auf nicht umlagefähige Nebenkosten von nur rund 92 Prozent eines Vergleichs- oder Durchschnittswertes hindeutet. Für diese ergibt sich ein jährlicher Wert von 768,18 EUR. Der Betrag nicht umlagefähiger Kosten (ohne Instandhaltungsaufwand) wird nicht angepaßt, weil der durchschnittliche Jahreswert von 363,52 EUR aus Vergangenheitswerten des Bewertungsobjekts gewonnen wurde. In Summe ergibt sich ein zu erwartender Zahlungsstrom in Höhe von 3.536,10 EUR jährlich, wie folgende Rechnung zeigt:

Mietertrag:

$$
5,92 \mathrm{EUR} \cdot 72,56 \mathrm{qm} \cdot 11,06 \text { Monate } \cdot 101,23 \% \quad=4.810,95 \mathrm{EUR}
$$

Umlagefähige Betriebskosten (bei Leerstand):

$$
-1.830,07 \text { EUR } \quad \cdot \frac{0,94}{12} \text { Monate } \quad=-143,15 \text { EUR }
$$

Nicht umlagefähige Nebenkosten (ohne Instandhaltung):

$$
-363,52 \mathrm{EUR}=-363,52 \mathrm{EUR}
$$

Nicht umlagefähige Nebenkosten (nur Instandhaltung):

$$
-11,50 \text { EUR }
$$$$
\cdot 72,56 \mathrm{qm}
$$

$\cdot 92,06 \%$

$\equiv-768,18 \mathrm{EUR}$

\section{Zahlungsstrom:}

$=3.536,10 \mathrm{EUR}$

Beide Nutzwerte weisen mit 2,12 bzw. 2,15 von maximal 4,00 Punkten ein leicht überdurchschnittliches Niveau auf. Dennoch wundert es nicht, daß die erwarteten jährlichen Zahlungsüberschüsse um knapp 400 EUR niedriger sind als in der vorangegangenen durchschnittlichen Berechnung. Dort wurde schließlich von vollen zwölf Monaten ohne Leerstand ausgegangen. Daß es sich um einen unrealistischen Wert handelte, war dem Investor bereits klar. Ähnliche Überlegungen führten ja gerade zur Notwendigkeit, eine Nutzwertanalyse zur Anpassung der Daten zu verwenden. Insoweit handelte es sich zuvor um eine unfertige Datenbasis, die nun verbessert bzw. vervollständigt wurde.

\subsection{Zahlungen am Planungshorizont}

Wie bereits erwähnt, geht der Investor von einer 50-jährigen Nutzungsdauer der Eigentumswohnung aus und unterteilt den Planungshorizont in eine zehnjährige Detailplanungsphase, gefolgt von einer vierzigjährigen Grobplanungsphase. ${ }^{389}$ Die Zielvorstellung eines möglichst langfristigen und gleichmäßigen Zusatzeinkommens, auch bis ins

\footnotetext{
${ }^{388}$ Vgl. § 28 Abs. 1 Nr. 3 II. BV. Dieser Wert ist für Gebäude vorgesehen, die älter als 32 Jahre sind. Für Gebäude im Alter zwischen 22 und 32 Jahren nennt das Gesetz Kosten von 9 EUR pro qm, und bei Gebäuden, die jünger als 22 Jahre sind, beträgt der Wert 7,10 EUR, vgl. ebd.

${ }^{389}$ Vgl. Gliederungspunkt 2.3.1.
} 
Rentenalter hinein, widerspricht dem Gedanken, die Wohnung am Ende des Planungshorizonts zu verkaufen. ${ }^{390}$ Daher ist kein Restwert im Sinne eines fiktiven Verkaufserlöses anzusetzen, sondern der Barwert der Mietüberschüsse jenseits des Detailplanungshorizonts in $t=10$. Aufgrund der Abhängigkeit vom Entscheidungsfeld muß der Investor den Zinssatz zur Berechnung des Barwertfaktors schätzen und dabei auf die Verzinsung potentieller Grenzobjekte abstellen. ${ }^{391}$ Wie bereits in Abschnitt 2.3.1 erläutert, zieht er einen zweiprozentigen Zinssatz heran, daraus ergibt sich ein Barwertfaktor von

$$
\frac{1,02^{40}-1}{0,02 \cdot 1,02^{40}}=27,36 .
$$

Die gleichbleibenden periodischen Zahlungsüberschüsse, die man ab der elften Periode erwartet, sind zur Berechnung des Barwerts mit diesem Faktor zu multiplizieren. Der anzusetzende Restwert am Detailplanungshorizont beträgt somit 3.536,10 $\cdot 27,36=$ 96.747,70 EUR. Er repräsentiert alle Zahlungen ab der elften Periode und wird bereits in der zehnten Periode in Form einer zusätzlichen Einzahlung angesetzt. Weil die Eigentumswohnung einen uniformen Zahlungsstrom hervorruft, kann hierfür der Faktor $27,36+1=28,36$ angewendet werden.

\subsubsection{Entscheidungsfeld als Bewertungsrahmen}

Welche zusätzlichen Objekte, Handlungsoptionen und Restriktionen im Entscheidungsfeld des Bewertungssubjekts aus seiner Sicht bedeutsam sind, ist Gegenstand der folgenden Ausführungen. Die für Konsumzwecke verfügbaren Zahlungsüberschüsse $b_{t}$ des Investors entstammen seinem Einkommen aus nichtselbständiger Tätigkeit abzgl. Steuern, Sozialabgaben und Lebenshaltungskosten.

In der Beispielsituation wird von einem genau durchschnittlichen Bruttoeinkommen ausgegangen, welches laut dem Statistischen Bundesamt 52.044 EUR p.a. beträgt. ${ }^{392}$ Davon sind Steuern und Sozialabgaben in Höhe von 20.455,19 EUR p.a. abzuziehen. ${ }^{393}$ So wird ihm ein jährliches Nettoeinkommen von 31.588,81 EUR ausgezahlt. Gleichwohl steht ihm dieser Betrag noch nicht zur freien Verfügung, denn Privatpersonen können sich nur innerhalb bestimmter Grenzen für oder gegen Konsumausgaben entscheiden. Man spricht von Lebenshaltungskosten, welche der Investor noch von seinem Nettoeinkommen abziehen muß, um den frei konsumier- oder investierbaren Betrag zu

${ }^{390}$ Vgl. zu dieser Zielsetzung HELLERFORTH (2012), S. 5, LEIFELS/RAFFELHÜSCHEN (2014), S. 135, ROTTKE/EIBEL (2017), S. $836 \mathrm{f}$.

${ }^{391} \mathrm{Vgl}$. HAX (1964), S. 438, siehe auch Gliederungspunkt 2.2.3.3.4.

${ }^{392}$ Vgl. STATISTISCHES BUNDESAMT (2018a), S. 178, dort in Form eines Monatswerts in Höhe von 4.337 EUR. Es sei nochmals daran erinnert: Durchschnittswerte sagen alles und gleichzeitig nichts. Ein Einkommen, das für alle Leute gleichermaßen gilt, gibt es nicht. Die nachfolgend verwendeten Einnahmen und Ausgaben sind mathematische Mittelwerte, die in der Realität bedeutungslos sind, weil sie auf niemanden zutreffen.

${ }^{393}$ Berechnung vom 08.10.2018 für den Veranlagungszeitraum 2019, siehe Anhang 2. 
errechnen. Es fallen zwingend notwendige Ausgaben für Grundbedürfnisse wie Ernährung oder Kleidung an oder für Wohnraum, Strom- und Wasserversorgung. Der durchschnittliche Bundesbürger, der ein statistisches Fantasiekonstrukt im Sinne einer Jedermannfiktion $^{394}$ ist, gibt jedes Jahr 29.760,00 EUR für private Konsumausgaben aus. ${ }^{395}$ Zieht man diesen Wert vom Nettoeinkommen ab, verbleiben dem Investor jährlich 1.828,81 EUR zur freien Verwendung. Tabelle 16 faßt die Berechnung zusammen:

\begin{tabular}{|c|c|}
\hline Daten & Werte in EUR p.a. \\
\hline Einkommen, Brutto & $52.044,00$ \\
\hline \multicolumn{2}{|c|}{ (Zu versteuerndes Einkommen: 42.501) } \\
\hline Sozialabgaben & $-10.473,84$ \\
\hline Einkommensteuer & $-9.461,00$ \\
\hline Solidaritätszuschlag & $-520,35$ \\
\hline Einkommen, Netto & $31.588,81$ \\
\hline Lebenshaltungskosten & $-29.760,00$ \\
\hline Summe $b_{t}$ & $1.828,81$ \\
\hline
\end{tabular}

Tabelle 16: Umrechnung vom Brutto- zum verfügbaren Einkommen

Das Entscheidungsfeld umfaßt ferner die Möglichkeit zur zinslosen, einperiodigen Kassenhaltung in unbegrenzter Höhe: Von Sicherheitsbedenken abgesehen, ist es für Privatpersonen immer möglich, Geld von der Bank abzuheben und zu Hause zu horten, um Negativzinsen zu entgehen. ${ }^{396}$

Weiterhin existieren zwei Möglichkeiten zur jeweils einperiodigen Kreditaufnahme: Die Sparkasse räumt dem Investor eine Kreditlinie zur beliebigen Verwendung in Höhe von 40.000 EUR ein. Der Zinssatz sei laut CAPM bereits risikoadjustiert und müsse acht Prozent p.a. betragen. ${ }^{397}$ Dieses Finanzierungsobjekt sei als Kreditlinie A bezeichnet. Falls der Investor sich für den Kauf der Eigentumswohnung entscheidet, stehen ihm Mittel aus dem EZB-Sofortprogramm zur notwendigen Stabilisierung des europäischen Immobilienmarktes als vergünstigte, zweckgebundene Kreditlinie zur Verfügung. Auf ministeriale Anweisung des EZB-Präsidenten NAGYON DRÁGA ${ }^{398}$ verfügt die Kreditlinie

${ }^{394} \mathrm{Zu}$ diesem Begriff vgl. MATSCHKE (1979), S. 176, KÜNNEMANN (1985), S. 17, MATSCHKE/BRÖSEL (2013), S. 16.

395 Vgl. STATISTISCHES BUNDESAMT (2018a), S. 178.

${ }^{396}$ Dies gilt zumindest, solange es noch Bargeld gibt. Zur politisch forcierten Idee der Bargeldabschaffung, u.a. unter dem Vorwand der Kriminalitätsbekämpfung, vgl. BECK/PRINZ(2015), S. 515, KÖNIG (2017), S. 341, DIEM MEIER (2019), S. 10: Ohne Bargeld wären Sparer nicht mehr in der Lage, sich durch Kassenhaltung vor der schleichenden Enteignung durch Negativzinsen zu schützen.

$397 \mathrm{Zu}$ diesem vgl. SHARPE (1964), LINTNER (1965), MOSSIN (1966). Zu Kritik, insbesondere an den Modellprämissen vgl. HERING/BRÖSEL (2004), S. 938, HERING/OLBRICH/ROLLBERG (2010), S. 32, MATSCHKE/BRÖSEL (2013), S. 40 ff., HERING (2017), S. 303 ff., SCHILDBACH (2017), S. 261. „Welches ist die Kovarianz zwischen der Errichtung eines Werkes in Ungarn und dem DAX?", pointiert HERING (2017), S. 309. Zur Eignung des CAPM i.R.d. Argumentationsfunktion vgl. BRÖSEL/TOLL (2011), S. 262, HERING (2014), S. 314.

${ }^{398}$ Ungarisch: „Sehr teuer“ (Übers. d. Verf., aber auch „sehr wertvoll“). Der Name ist frei erfunden. 
über einen auffällig günstigen Zinssatz von nur einem Prozent p.a. Die Höhe des Kreditlinie B genannten Darlehens ist betragsmäßig auf den Kaufpreis der Immobilie begrenzt, maximal jedoch auf 450.000 EUR. ${ }^{399}$

Neben $b_{t}$ und den Möglichkeiten zur Geldanlage und Kreditaufnahme gehört auch ein weiteres Investitionsobjekt zum Entscheidungsfeld. An der örtlichen Wirtschaftsschule kann der Investor jedes Jahr im Rahmen einer freiberuflichen Tätigkeit Mathematik unterrichten. ${ }^{400}$ Sofern er sich zur Durchführung entscheidet, besteht eine jährliche Nachfrage von insgesamt 100 Schülern, die zur Zahlung einer Seminargebühr von jeweils 490 EUR bereit sind. Dieses Investitionsobjekt ist zweiperiodig: Im Rahmen der Vorbereitungen müssen bestimmte Auszahlungen getätigt werden: Die Herstellung der Seminarunterlagen kostet 200 EUR pro Teilnehmer, da neben Druckkosten auch Lizenzzahlungen für eine rechnergestützte Anwendung zu entrichten sind. Zusätzlich werden für jeden Teilnehmer anschauliche Vorbereitungsmaterialien für 90 EUR erstellt. Die Versandkosten betragen 9 EUR pro Teilnehmer. Der Investor muß also im Vorjahr der freiberuflichen Tätigkeit mit 299 EUR pro Teilnehmer in Vorleistung treten. Im Jahr der Durchführung sind 30 Prozent der Einnahmen, also 147 EUR pro Teilnehmer, als Servicegebühr an die Schule zu entrichten. Der Investor erzielt pro Teilnehmer einen Überschuß von 343 EUR, abzüglich seiner Vorleistung von 299 EUR im Vorjahr. Vereinfachend wird angenommen, daß die Rückflüsse pro Teilnehmer sicher sind und von der Schule garantiert werden. Zudem wird von der Umsatzsteuer abgesehen, weil diese ohnehin ein durchlaufender Posten ist. ${ }^{401}$ Das Investitionsobjekt Mathematikunterricht wird als beliebig teilbar angenommen, ${ }^{402}$ weil der Investor selbst über seine Kapazitätsauslastung entscheidet: Jede Konstellation zwischen null und 100 Teilnehmern ist möglich. Tabelle 17 faßt die Daten zusammen.

${ }^{399}$ Wie sich später in der exemplarischen Berechnung zeigen wird, ist diese Obergrenze viel zu hoch angesetzt. Aber das ist nebensächlich, schließlich dient der Kredit einem politisch forcierten Zweck.

${ }^{400}$ Für das Beispiel wurde bewußt keine gewerbliche Nebentätigkeit ins Feld geführt, damit keine gewerbesteuerliche Betrachtung erforderlich ist. Die höhere Komplexität würde im Ausgangsbeispiel von der eigentlichen Betrachtung ablenken.

${ }^{401}$ Zur Umsatzsteuer vgl. KUßMAUL (2016), S. 476 ff., SCHREIBER (2017), S. 169 ff., SCHNEELOCH/ MEYERING/PATEK (2017b), S. 61 ff., KRAFT/KRAFT (2018), S. 315 ff., NICKENIG (2018), S. 67 ff.

${ }^{402} \mathrm{Zu}$ unteilbaren Objekten und daraus folgenden Ganzzahligkeitsproblemen vgl. HAX (1964), S. 442, HERING (2017), S. 202 ff. In diesen Fällen kann es passieren, daß mangels anteilig durchgeführtem Grenzobjekt auch ex post keine periodenspezifischen Steuerungszinsen existieren und die Kapitalwertmethode versagt, vgl. dazu HAX (1985), S. 98, KLINGELHÖFER (2006), S. 42. 


\begin{tabular}{|l|r|r|r|}
\hline Freiberufliche Tätigkeit (Mathematikunterricht) \\
\hline Zeitpunkt t: & & & \\
\hline Herstellung Seminarunterlagen & $-200,00$ & x 100 Stück $=$ & $-20.000,00$ \\
\hline Herstellung Vorbereitungsmaterial & $-90,00$ & x 100 Stück $=$ & $-9.000,00$ \\
\hline Versandkosten & $-9,00$ & x 100 Stück $=$ & $-900,00$ \\
\hline Summe Zeitpunkt t: & $-\mathbf{2 9 9 , 0 0}$ & x 100 Stück $=$ & $-\mathbf{2 9 . 9 0 0 , 0 0}$ \\
\hline Zeitpunkt t+1: & & & \\
\hline Seminargebühr & 490,00 & x 100 Teilnehmer $=$ & $49.000,00$ \\
\hline Servicepauschale (30\%) & $-147,00$ & x 100 Teilnehmer $=$ & $-14.700,00$ \\
\hline Summe Zeitpunkt t+1: & $\mathbf{3 4 3 , 0 0}$ & x 100 Teilnehmer $=$ & $\mathbf{3 4 . 3 0 0 , 0 0}$ \\
\hline
\end{tabular}

Tabelle 17: Investitionsobjekt Mathematikunterricht

Die mehrperiodigen Objekte des Entscheidungsfelds sind in Tabelle 18, die einperiodigen Objekte in Tabelle 19 zusammengefaßt.

\begin{tabular}{|l|r|r|r|r|l|}
\hline Objekt & Symbol & $\mathbf{t}=\mathbf{0}$ & $\mathbf{t}=\mathbf{1}$ & $\mathbf{t}=\mathbf{2}$ & $\mathbf{t}=\mathbf{3}$ \\
\hline Feste Zahlungen: & $\mathrm{b}_{\mathrm{t}}$ & 0 & $1.828,81$ & $1.828,81$ & $\ldots$ \\
\hline Wohnung: & $\mathrm{g}_{\mathrm{Kt}}$ & $-\mathrm{p}$ & $3.536,10$ & $3.536,10$ & $\ldots$ \\
\hline
\end{tabular}

Tabelle 18: Mehrperiodige Objekte

\begin{tabular}{|l|r|r|r|r|r|r|}
\hline Objekt & Symbol & $\mathbf{t}-1$ & $\mathbf{t}$ & $\mathbf{t}+\mathbf{1}$ & $\mathbf{t}+\mathbf{2}$ & Obergrenze \\
\hline Freiberufliche Tätigkeit: & $\mathrm{I}_{\mathrm{t}}$ & - & -29.900 & 34.300 & - & 1 \\
\hline Kassenhaltung: & $\mathrm{G}_{\mathrm{t}}$ & - & $-1,00$ & 1,00 & - & $\infty$ \\
\hline Kreditlinie A: & $\mathrm{A}_{\mathrm{t}}$ & - & 1,00 & $-1,08$ & - & 40.000 \\
\hline Kreditlinie B: & $\mathrm{B}_{\mathrm{t}}$ & - & 1,00 & $-1,01$ & - & $\max .\{\mathrm{p} ; 450.000\}$ \\
\hline
\end{tabular}

Tabelle 19: Einperiodige Objekte

\subsubsection{Zustands-Grenzpreismodell als anwendbares Totalmodell}

\subsubsection{Ermittlung des optimalen Basisprogramms ohne Eigentumswohnung}

Die zusammengestellten Daten werden nun in die Form eines linearen Optimierungsproblems gebracht. Zuerst wird der Ansatz zur Ermittlung des Basisprogramms ohne Bewertungsobjekt aufgestellt. Für den Fall der Einkommensmaximierung ergibt sich das folgende Ungleichungssystem:

max. Entn; Entn $:=$ EN

$$
\begin{array}{lll}
+29.900 \mathrm{I}_{0} & +1 \mathrm{G}_{0}-1 \mathrm{~A}_{0} & \leq 0,00 \\
+29.900 \mathrm{I}_{1}-34.300 \mathrm{I}_{0}+1 \mathrm{G}_{1}-1 \mathrm{G}_{0}-1 \mathrm{~A}_{1}+1,08 \mathrm{~A}_{0}+\mathrm{EN} & \leq 1.828 \\
+29.900 \mathrm{I}_{2}-34.300 \mathrm{I}_{1}+1 \mathrm{G}_{2}-1 \mathrm{G}_{1}-1 \mathrm{~A}_{2}+1,08 \mathrm{~A}_{1}+\mathrm{EN} & \leq 1.828 \\
+29.900 \mathrm{I}_{3}-34.300 \mathrm{I}_{2}+1 \mathrm{G}_{3}-1 \mathrm{G}_{2}-1 \mathrm{~A}_{3}+1,08 \mathrm{~A}_{2}+\mathrm{EN} & \leq 1.828 \\
+29.900 \mathrm{I}_{4}-34.300 \mathrm{I}_{3}+1 \mathrm{G}_{4}-1 \mathrm{G}_{3}-1 \mathrm{~A}_{4}+1,08 \mathrm{~A}_{3}+\mathrm{EN} & \leq 1.828 \\
+29.900 \mathrm{I}_{5}-34.300 \mathrm{I}_{4}+1 \mathrm{G}_{5}-1 \mathrm{G}_{4}-1 \mathrm{~A}_{5}+1,08 \mathrm{~A}_{4}+\mathrm{EN} & \leq 1.828 \\
+29.900 \mathrm{I}_{6}-34.300 \mathrm{I}_{5}+1 \mathrm{G}_{6}-1 \mathrm{G}_{5}-1 \mathrm{~A}_{6}+1,08 \mathrm{~A}_{5}+\mathrm{EN} & \leq 1.828 \\
+29.900 \mathrm{I}_{7}-34.300 \mathrm{I}_{6}+1 \mathrm{G}_{7}-1 \mathrm{G}_{6}-1 \mathrm{~A}_{7}+1,08 \mathrm{~A}_{6}+\mathrm{EN} & \leq 1.828
\end{array}
$$




$$
\begin{aligned}
& +29.900 \mathrm{I}_{8}-34.300 \mathrm{I}_{7}+1 \mathrm{G}_{8}-1 \mathrm{G}_{7}-1 \mathrm{~A}_{8}+1,08 \mathrm{~A}_{7}+\mathrm{EN} \leq 1.828 \\
& +29.900 \mathrm{I}_{9}-34.300 \mathrm{I}_{8}+1 \mathrm{G}_{9}-1 \mathrm{G}_{8}-1 \mathrm{~A}_{9}+1,08 \mathrm{~A}_{8}+\mathrm{EN} \leq 1.828 \\
& -34.300 \mathrm{I}_{9} \quad-1 \mathrm{G}_{9} \quad+1,08 \mathrm{~A}_{9}+28,36 \cdot \mathrm{EN} \leq 28,36 \cdot 1.828 \\
& 0 \leq G_{0}, G_{1}, G_{2}, G_{3}, G_{4}, G_{5}, G_{6}, G_{7}, G_{8}, G_{9} \\
& 0 \leq \mathrm{I}_{0}, \mathrm{I}_{1}, \mathrm{I}_{2}, \mathrm{I}_{3}, \mathrm{I}_{4}, \mathrm{I}_{5}, \mathrm{I}_{6}, \mathrm{I}_{7}, \mathrm{I}_{8}, \mathrm{I}_{9} \leq 1 \\
& 0 \leq A_{0}, A_{1}, A_{2}, A_{3}, A_{4}, A_{5}, A_{6}, A_{7}, A_{8}, A_{9} \leq 40.000 \\
& 0 \leq \mathrm{EN}
\end{aligned}
$$

Die erste Zeile ist die Zielfunktion. Sie vermittelt dem Algorithmus, daß EN als periodisch wiederkehrende Entnahme die zu maximierende Zielgröße ist. Die nächsten elf Zeilen sind Liquiditätsnebenbedingungen und umfassen neben den zehn Zeiträumen innerhalb des Detailplanungshorizonts auch $\mathrm{t}=0$ als Zeitpunkt der Entscheidung. Gleichwohl haben alle Perioden t den Charakter von Zeitpunkten, weil die Investitionstheorie Zahlungen auf das jeweilige Periodenende bezieht. ${ }^{403} \mathrm{Da} t=0$ gedanklich den 31. Dezember des Vorjahres verkörpert, verwundert es nicht, daß in $t=0$ weder eine Entnahme noch ein frei verfügbares Einkommen zu finden ist. ${ }^{404}$ Die einperiodigen Objekte $I_{t}$ (Mathematikunterricht), $G_{t}$ (Geldanlage) und $A_{t}$ (Kreditlinie A) sind entsprechend dem Beginn ihrer Durchführung mit dem Zeitindex $t$ gekennzeichnet. Wird beispielsweise in $\mathrm{t}=5$ für eine Periode bis $\mathrm{t}=6$ Kassenhaltung betrieben, wird dieser Vorgang durch die Variable $G_{5}$ ausgedrückt. Die Liquiditätsnebenbedingungen besagen, daß in jeder Periode die Einzahlungen aus freiberuflicher Tätigkeit, Rückzahlung der Geldanlage, Aufnahme eines Kredits sowie aus dem frei verfügbaren Einkommen mindestens genauso hoch sein müssen wie die Auszahlungen für die Vorbereitung der nächsten freiberuflichen Tätigkeit, Kreditrückzahlungen, Geldanlagen sowie Konsumentnahmen. Die Liquiditätsnebenbedingung der letzten Periode unterscheidet sich von den vorherigen: Hier enden die Geldanlage-, Investitions- und Kreditaufnahmeobjekte - und mangels Folgeperiode stehen keine neuen Objekte zur Verfügung. Unabhängig von deren Verfügbarkeit erfolgt die Fortrechnung sowohl des frei verfügbaren Einkommens als auch der Entnahmen über den Planungshorizont hinaus mit Hilfe des Faktors 28,36. Dieser erzeugt für beide Größen den Barwert der folgenden 40 Jahre bei einem Zinssatz von zwei Prozent. ${ }^{405}$ Weil dieser Zinssatz bereits die Grenzverwendung des Geldes jenseits des Detailplanungshorizonts abbildet, sind keine weiteren Grenzobjekte mehr erforderlich; mit dem geschätzten Kalkulationszinsfuß wurde bereits ein fiktives Grenzobjekt erschaffen. ${ }^{406}$

\footnotetext{
${ }^{403}$ Vgl. HAX (1985), S. 11, TER HORST (2009), S. 38, BITZ/EWERT/TERSTEGE (2018), S. 8. ${ }^{404}$ Vgl. Hering (2014), S. 38, HeRING/TOLL (2017a), S. 176, BUSSE VON COLBE/WitTE (2018), S. 12.

${ }^{405}$ Wie bereits erwähnt, bezieht sich nur sein Anteil in Höhe von 27,36 als Rentenbarwertfaktor auf alle 40 Jahre jenseits des Planungshorizonts. Der Faktor eins steht für die ebenfalls zu erfassenden Elemente, die zur zehnten Periode gehören.

${ }^{406}$ Fraglich ist, ob der Zeitraum von 50 Jahren gleichermaßen für die Immobilie wie für das verfügbare Einkommen heranzuziehen ist. Das Erwerbsleben eines Erwachsenen umfaßt maximal 49 Jahre,
} 
Die letzten vier Zeilen stellen Obergrenzen- bzw. Nichtnegativitätsbedingungen dar. Sie wurden aus Gründen der besseren Lesbarkeit komprimiert dargestellt. Jede Zeile bezieht sich auf eine Objektklasse. Die Geldanlagen der Perioden null bis neun sind nichtnegativ und verfügen über keine Obergrenze. Gleiches gilt für die Konsumentnahmen EN, schließlich handelt es sich um die Zielgröße, die möglichst maximiert werden soll. Anders das Investitionsobjekt Mathematikunterricht, dessen beliebig teilbare Durchführungshäufigkeit zwischen null und eins liegt, wobei die eins für eine vollständige Durchführung mit 100 Studenten steht. Das Kreditobjekt A ist ebenfalls nichtnegativ und auf maximal 40.000 EUR beschränkt. Die Bedeutung der letzten Zeilen sei nochmals beispielhaft verdeutlicht: Der von einem Objekt hervorgerufene Zahlungsstrom errechnet sich durch Multiplikation der Variable, z.B. $A_{0}$, mit ihrer Durchführungshäufigkeit, z.B. $x_{A_{0}}$. Wenn Kreditlinie $A$ in $t=0$ in maximaler Höhe beansprucht wird, kommt es in $\mathrm{t}=0 \mathrm{zu}$ einer Einzahlung von $1 \cdot \mathrm{A}_{0}=1 \cdot 40.000=40.000$. In $\mathrm{t}=1 \mathrm{mu} ß \mathrm{der}$ einperiodige Kredit $A_{0}$ zurückgezahlt werden, entsprechend einem Betrag von $1,08 \cdot A_{0}=$ $1,08 \cdot 40.000=43.200$ EUR. Es darf nicht täuschen, daß in $\mathrm{t}=1$ erneut ein identisch ausgestattetes, aber hiervon unabhängiges Objekt $\mathrm{A}_{1}$ zur Kreditaufnahme zur Verfügung steht, ebenso in der nächsten Periode, usw.

Zur Lösung des linearen Optimierungsansatzes stehen verschiedene Programme für gewöhnliche Arbeitsplatzrechner zur Verfügung, die teils sogar kostenfrei sind, z.B. der bei Microsoft Excel mitgelieferte Solver oder das Programm Matlab. ${ }^{407}$ In der vorliegenden Arbeit wurde das Programm Lingo zur Lösung herangezogen. Aus der Lösung wird ersichtlich, daß der Investor jedes Jahr eine Konsumentnahme von 2.523 EUR tätigen kann. Der Mathematikunterricht wird in allen Perioden vollständig durchgeführt. Mangels Anfangsvermögen wird dessen Investitionsauszahlung zu Anfang vollständig durch Kreditlinie A finanziert. Diese hat eine Obergrenze von 40.000 EUR, wird aber nur anteilig in Anspruch genommen und ist somit Grenzobjekt. Die zinslose Kassenhaltung wird zu keinem Zeitpunkt in Anspruch genommen, weil eine Kreditaufnahme und Investition in die Lehrtätigkeit lohnenswerter ist.

wenn er mit 18 Jahren zu arbeiten beginnt und mit 67 Jahren in den Ruhestand geht. Das Renteneintrittsalter dürfte i.d.R. nicht erst 49 Jahre nach Investitionsbeginn zu erwarten sein, weil die wenigsten Vermieter bereits mit 18 Jahren zu investieren beginnen; private Vermieter sind deutlich älter als die Gesamtbevölkerung, knapp ein Drittel ist über 65 Jahre alt, vgl. INSTITUT DER DEUTSCHEN WIRTSCHAFT KÖLN (2017), S. 4. Im Rentenalter verändert sich die Höhe des frei verfügbaren Einkommens kaum, weil nicht nur Einnahmen, sondern auch Ausgaben sinken, z.B. Fahrten zur Arbeit oder Mittagessen außer Haus. Sicherlich könnte man beim Einkommen einen anderen Barwertfaktor ansetzen, um den verbleibenden Jahren im Arbeitsleben besser gerecht zu werden. Doch gehört es zu den Aufgaben der Investitionstheorie, zu Vereinfachungszwecken Komplexitätsreduktionen und vereinfachende Annahmen zu treffen, damit die Rechnung nicht ausufert. Ziel des Modells ist, ,problemadäquat entscheiden zu können“, BALLWIESER (1990), S. 12. Schließlich geht der Entscheidungswert aus dem Vergleich von Basis- und Bewertungsprogramm unter gleichen Bedingungen hervor.

${ }^{407}$ Einen Überblick über rechnergestützte Programme zur (linearen) Optimierung findet man bei SUHL/MELLOULI (2013), S. 77 ff., WERNERS (2013), S. 123 ff., DOMSCHKE/DREXL/KLEIN/SCHOLL (2015), S. 14 ff. 
Tabelle 20 stellt das optimale Basisprogramm als vollständigen Finanzierungsplan (VOFI) dar. ${ }^{408}$ Die Nachkommastellen aller in der optimalen Lösung vorkommenden Variablen werden zur besseren Lesbarkeit hier und im weiteren Verlauf der Arbeit kaufmännisch gerundet. Außerdem wurden Investition, Geldanlage und Kreditaufnahme in mehreren Zeilen dargestellt, damit deren Tilgung und Zinszahlungen besser erkennbar sind. Die vorletzte Zeile zeigt die Summe aller Zahlungsströme der jeweiligen Periode und läßt die Einhaltung der Liquiditätsnebenbedingungen erkennen. Außerdem erkennt man in der als Konto(-stand) bezeichneten letzten Zeile den summierten Guthabensbzw. Schuldenstand aller Geldanlage- und Kreditobjekte.

\begin{tabular}{|c|c|c|c|c|c|c|c|c|c|c|c|}
\hline & $\mathrm{t}=0$ & $\mathrm{t}=1$ & $\mathrm{t}=2$ & $\mathrm{t}=3$ & $\mathrm{t}=4$ & $\mathrm{t}=5$ & $\mathrm{t}=6$ & $\mathrm{t}=7$ & $\mathrm{t}=8$ & $\mathrm{t}=9$ & $\begin{array}{c}\mathrm{t}=10 \& \\
\text { Barwert }\end{array}$ \\
\hline $\mathrm{EN}$ & - & -2523 & -2523 & -2523 & -2523 & -2523 & -2523 & -2523 & -2523 & -2523 & -71556 \\
\hline $\mathrm{I}_{\mathrm{t}}$ & -29900 & -29900 & -29900 & -29900 & -29900 & -29900 & -29900 & -29900 & -29900 & -29900 & 0 \\
\hline $\mathrm{I}_{\mathrm{t}-1}$ & 0 & 34300 & 34300 & 34300 & 34300 & 34300 & 34300 & 34300 & 34300 & 34300 & 34300 \\
\hline $\mathrm{G}_{\mathrm{t}}$ & 0 & 0 & 0 & 0 & 0 & 0 & 0 & 0 & 0 & 0 & - \\
\hline $\mathrm{G}_{\mathrm{t}-1}$ & - & 0 & 0 & 0 & 0 & 0 & 0 & 0 & 0 & 0 & 0 \\
\hline $\mathrm{A}_{\mathrm{t}}$ & 29900 & 28587 & 27169 & 25638 & 23984 & 22198 & 20269 & 18186 & 15936 & 13506 & - \\
\hline $\mathrm{A}_{\mathrm{t}-1}$ & - & -29900 & -28587 & -27169 & -25638 & -23984 & -22198 & -20269 & -18186 & -15936 & -13506 \\
\hline $\mathrm{A}($ Zins $)$ & - & -2392 & -2287 & -2174 & -2051 & -1919 & -1776 & -1622 & -1455 & -1275 & -1080 \\
\hline $\mathrm{B}_{\mathrm{t}}$ & - & - & - & - & - & - & - & - & - & - & - \\
\hline $\mathrm{B}_{\mathrm{t}-1}$ & - & - & - & - & - & - & - & - & - & - & - \\
\hline $\mathrm{B}($ Zins $)$ & - & - & - & - & - & - & - & - & - & - & - \\
\hline $\mathrm{b}_{\mathrm{t}}$ & - & 1828 & 1828 & 1828 & 1828 & 1828 & 1828 & 1828 & 1828 & 1828 & 51842 \\
\hline$\sum$ & 0 & 0 & 0 & 0 & 0 & 0 & 0 & 0 & 0 & 0 & 0 \\
\hline Konto & -29900 & -28587 & -27169 & -25638 & -23984 & -22198 & -20269 & -18186 & -15936 & -13506 & 0 \\
\hline
\end{tabular}

Tabelle 20: VOFI des optimalen Basisprogramms

\subsubsection{Ermittlung des optimalen Bewertungsprogramms einschließlich Eigentumswohnung}

Durch Hinzufügen des Zahlungsstroms der Eigentumswohnung sowie der immobilienbezogenen Kreditobjekte wird nun der Ansatz zur Ermittlung des optimalen Bewertungsprogramms aufgestellt. Veränderungen zum Basisprogramm sind zur besseren Erkennbarkeit blau hervorgehoben.

$\max . \mathrm{U} ; \mathrm{U}:=\mathrm{p}$

$29900 \mathrm{I}_{0}+1 \mathrm{G}_{0}-1 \mathrm{~A}_{0}-1 \mathrm{~B}_{0} \quad+\mathrm{p} \leq 0$
$29900 \mathrm{I}_{1}-34300 \mathrm{I}_{0}+1 \mathrm{G}_{1}-1 \mathrm{G}_{0}-1 \mathrm{~A}_{1}+1,08 \mathrm{~A}_{0}-1 \mathrm{~B}_{1}+1,01 \mathrm{~B}_{0}+\mathrm{EN} \leq 1828+3536,1$
$29900 \mathrm{I}_{2}-34300 \mathrm{I}_{1}+1 \mathrm{G}_{2}-1 \mathrm{G}_{1}-1 \mathrm{~A}_{2}+1,08 \mathrm{~A}_{1}-1 \mathrm{~B}_{2}+1,01 \mathrm{~B}_{1}+\mathrm{EN} \leq 1828+3536,1$
$29900 \mathrm{I}_{3}-34300 \mathrm{I}_{2}+1 \mathrm{G}_{3}-1 \mathrm{G}_{2}-1 \mathrm{~A}_{3}+1,08 \mathrm{~A}_{2}-1 \mathrm{~B}_{3}+1,01 \mathrm{~B}_{2}+\mathrm{EN} \leq 1828+3536,1$
$29900 \mathrm{I}_{4}-34300 \mathrm{I}_{3}+1 \mathrm{G}_{4}-1 \mathrm{G}_{3}-1 \mathrm{~A}_{4}+1,08 \mathrm{~A}_{3}-1 \mathrm{~B}_{4}+1,01 \mathrm{~B}_{3}+\mathrm{EN} \leq 1828+3536,1$

${ }^{408}$ Zur Verwendung von vollständigen Finanzplänen zur transparenten Abbildung von Immobilieninvestitionen vgl. HELLERFORTH (2008), S. 28, SCHULTE/SOTELO/ALLENDORF/ROPETER-AHLERS/ $L A N G$ (2016), S. 604 ff. 


$$
\begin{aligned}
& 29900 I_{5}-34300 I_{4}+1 G_{5}-1 G_{4}-1 A_{5}+1,08 A_{4}-1 B_{5}+1,01 B_{4}+E N \leq 1828+3536,1 \\
& 29900 \mathrm{I}_{6}-34300 \mathrm{I}_{5}+1 \mathrm{G}_{6}-1 \mathrm{G}_{5}-1 \mathrm{~A}_{6}+1,08 \mathrm{~A}_{5}-1 \mathrm{~B}_{6}+1,01 \mathrm{~B}_{5}+\mathrm{EN} \leq 1828+3536,1 \\
& 29900 I_{7}-34300 I_{6}+1 G_{7}-1 G_{6}-1 A_{7}+1,08 A_{6}-1 B_{7}+1,01 B_{6}+E N \leq 1828+3536,1 \\
& 29900 I_{8}-34300 I_{7}+1 G_{8}-1 G_{7}-1 A_{8}+1,08 A_{7}-1 B_{8}+1,01 B_{7}+E N \leq 1828+3536,1 \\
& 29900 \mathrm{I}_{9}-34300 \mathrm{I}_{8}+1 \mathrm{G}_{9}-1 \mathrm{G}_{8}-1 \mathrm{~A}_{9}+1,08 \mathrm{~A}_{8}-1 \mathrm{~B}_{9}+1,01 \mathrm{~B}_{8}+\mathrm{EN} \leq 1828+3536,1 \\
& \begin{array}{llll}
-34300 \mathrm{I}_{9} & -1 \mathrm{G}_{9}+1,08 \mathrm{~A}_{9} & +1,01 \mathrm{~B}_{9}+28,36 \mathrm{EN}
\end{array} \\
& \leq 28,36 \cdot(1828+3536,1) \\
& -\mathrm{EN} \leq-\mathrm{EN}^{*} \\
& 0 \leq \mathrm{G}_{0}, \mathrm{G}_{1}, \mathrm{G}_{2}, \mathrm{G}_{3}, \mathrm{G}_{4}, \mathrm{G}_{5}, \mathrm{G}_{6}, \mathrm{G}_{7}, \mathrm{G}_{8}, \mathrm{G}_{9} \\
& 0 \leq \mathrm{I}_{0}, \mathrm{I}_{1}, \mathrm{I}_{2}, \mathrm{I}_{3}, \mathrm{I}_{4}, \mathrm{I}_{5}, \mathrm{I}_{6}, \mathrm{I}_{7}, \mathrm{I}_{8}, \mathrm{I}_{9} \leq 1 \\
& 0 \leq A_{0}, A_{1}, A_{2}, A_{3}, A_{4}, A_{5}, A_{6}, A_{7}, A_{8}, A_{9} \leq 40.000 \\
& 0 \leq \mathrm{B}_{0}, \mathrm{~B}_{1}, \mathrm{~B}_{2}, \mathrm{~B}_{3}, \mathrm{~B}_{4}, \mathrm{~B}_{5}, \mathrm{~B}_{6}, \mathrm{~B}_{7}, \mathrm{~B}_{8}, \mathrm{~B}_{9} \leq \mathrm{p} \\
& 0 \leq \mathrm{B}_{0}, \mathrm{~B}_{1}, \mathrm{~B}_{2}, \mathrm{~B}_{3}, \mathrm{~B}_{4}, \mathrm{~B}_{5}, \mathrm{~B}_{6}, \mathrm{~B}_{7}, \mathrm{~B}_{8}, \mathrm{~B}_{9} \leq 450.000 \\
& 0 \leq \mathrm{EN} \\
& 0 \leq \mathrm{p}
\end{aligned}
$$

Der zu maximierende Zielfunktionswert ist nicht mehr das Einkommen, sondern der Kaufpreis der Eigentumswohnung, ausgedrückt durch die Variable p. Er ist in $\mathrm{t}=0 \mathrm{zu}$ entrichten und in der Liquiditätsnebenbedingung des Entscheidungszeitpunktes erfaßt und konsequenterweise auch in den Nichtnegativitätsbedingungen zu finden. Unverändert findet man die Objekte I (Mathematikunterricht), G (Geldanlage) und A (Kreditlinie A) in den Liquiditätsnebenbedingungen, jedoch tritt die zweckgebundene Immobilien-Kreditlinie als Objekt B hinzu. Die Höhe ist betragsmäßig auf den Kaufpreis der Eigentumswohnung, maximal jedoch auf 450.000 EUR begrenzt. Der aus der Eigentumswohnung erwartete Zahlungsstrom in Höhe von 3.536,10 EUR wurde auf den rechten Seiten der Ungleichungen zum feststehenden Einkommen $b_{t}$ addiert und findet auch über den Detailplanungshorizont hinaus als Barwert Berücksichtigung. Weil die Entnahmen EN nicht mehr Zielgröße sind, wurde eine Nebenbedingung ergänzt, die fordert, ihren optimalen Wert EN* aus dem Basisprogramm nicht zu unterschreiten.

Tabelle 21 stellt die im optimalen Bewertungsprogramm vorkommenden Objekte dar. Der Entscheidungswert der Eigentumswohnung liegt bei 127.728 EUR und wird vollständig über die Immobilienkreditlinie B finanziert. Es sei daran erinnert, daß der Grenzpreis auch Erwerbsnebenkosten umfaßt, nämlich die 6,5-prozentige Grunderwerbsteuer und etwa 1,5 Prozent Notar- und Grundbuchkosten. Weil der Verkäufer die Transaktionskosten nicht erhält, muß der präsumtive Käufer für die Verhandlung auch den reinen Kauf(grenz)preis kennen. Diesen ermittelt er, indem er den Grenzpreis durch den Faktor 1,08 dividiert. ${ }^{409}$ Seine Verhandlungsgrenze für den reinen Immobilienpreis beträgt somit 118.266,67 EUR.

Das Investitionsobjekt Mathematikunterricht ist auch im Bewertungsprogramm vorteilhaft und wird in allen Perioden vollständig durchgeführt. Kreditlinie A stellt auch hier die nötigen Mittel zur Verfügung, weil Kreditlinie B auf die Höhe des Immobilienkauf-

${ }^{409} \mathrm{Vgl}$. Gliederungspunkt 2.2.3.3.1. 
preises beschränkt ist. Die vorletzte Zeile beweist erneut, daß Liquiditätsnebenbedingungen aller Zeitpunkte eingehalten werden und sich die Ein- und Auszahlungen stets zu null EUR summieren.

\begin{tabular}{|c|c|c|c|c|c|c|c|c|c|c|c|}
\hline & $\mathrm{t}=0$ & $\mathrm{t}=1$ & $\mathrm{t}=2$ & $\mathrm{t}=3$ & $\mathrm{t}=4$ & $\mathrm{t}=5$ & $\mathrm{t}=6$ & $\mathrm{t}=7$ & $\mathrm{t}=8$ & $\mathrm{t}=9$ & $\begin{array}{c}\mathrm{t}=10 \text { \& } \\
\text { Barwert }\end{array}$ \\
\hline $\mathrm{p}$ & -127728 & - & - & - & - & - & - & - & - & - & - \\
\hline $\mathrm{EN}$ & - & -2523 & -2523 & -2523 & -2523 & -2523 & -2523 & -2523 & -2523 & -2523 & -71556 \\
\hline $\mathrm{g}_{\mathrm{Kt}}$ & 0 & 3536 & 3536 & 3536 & 3536 & 3536 & 3536 & 3536 & 3536 & 3536 & 100284 \\
\hline $\mathrm{I}_{\mathrm{t}}$ & -29900 & -29900 & -29900 & -29900 & -29900 & -29900 & -29900 & -29900 & -29900 & -29900 & - \\
\hline $\mathrm{I}_{\mathrm{t}-1}$ & - & 34300 & 34300 & 34300 & 34300 & 34300 & 34300 & 34300 & 34300 & 34300 & 34300 \\
\hline $\mathrm{G}_{\mathrm{t}}$ & 0 & 0 & 0 & 0 & 0 & 0 & 0 & 0 & 0 & 0 & - \\
\hline $\mathrm{G}_{\mathrm{t}-1}$ & - & 0 & 0 & 0 & 0 & 0 & 0 & 0 & 0 & 0 & 0 \\
\hline $\mathrm{A}_{\mathrm{t}}$ & 29900 & 26328 & 22471 & 18305 & 13806 & 8946 & 3698 & 0 & 0 & 0 & - \\
\hline $\mathrm{A}_{\mathrm{t}-1}$ & - & -29900 & -26328 & -22471 & -18305 & -13806 & -8946 & -3698 & 0 & 0 & 0 \\
\hline $\mathrm{A}($ Zins $)$ & - & -2392 & -2106 & -1798 & -1464 & -1104 & -716 & -296 & 0 & 0 & 0 \\
\hline $\mathrm{B}_{\mathrm{t}}$ & 127728 & 127728 & 127728 & 127728 & 127728 & 127728 & 127728 & 125759 & 119776 & 113732 & - \\
\hline $\mathrm{B}_{\mathrm{t}-1}$ & - & -127728 & -127728 & -127728 & -127728 & -127728 & -127728 & -127728 & -125759 & -119776 & -113732 \\
\hline $\mathrm{B}($ Zins $)$ & - & -1277 & -1277 & -1277 & -1277 & -1277 & -1277 & -1277 & -1258 & -1198 & -1137 \\
\hline $\mathrm{b}_{\mathrm{t}}$ & - & 1828 & 1828 & 1828 & 1828 & 1828 & 1828 & 1828 & 1828 & 1828 & 51842 \\
\hline$\sum$ & 0 & 0 & 0 & 0 & 0 & 0 & 0 & 0 & 0 & 0 & 0 \\
\hline Konto & -157628 & -154057 & -150199 & -146033 & -141534 & -136675 & -131427 & -125759 & -119776 & -113732 & 0 \\
\hline
\end{tabular}

Tabelle 21: VOFI des optimalen Bewertungsprogramms

Interessant ist die Wechselwirkung der beiden Kreditobjekte A und B: Zwar ist Kreditlinie B als zweckgebundener Immobilienkredit auf die Höhe des Kaufpreises begrenzt. Daher ist die Inanspruchnahme der deutlich teureren Kreditlinie A für die Durchführung des Mathematikunterrichts erforderlich. Weil der Algorithmus die finanziellen Mittel ihrer lohnendsten Verwendung zuleitet und im Modell keine Tilgungsmodalitäten, insbesondere keine jährliche Mindesttilgung erfaßt wurden, wird zunächst nur die teure Kreditlinie A so schnell wie möglich getilgt. Erst als in $t=7$ nach deren Abschlußtilgung in Höhe von 3.698 EUR noch Geld übrig ist, wird mit der Tilgung der Immobilienkreditlinie B überhaupt erst begonnen. Die Tilgung der Kreditlinie A stellt also auch im Bewertungsprogramm erneut die beste Verwendung des letzten verfügbaren Euro dar und ist bis $t=6$ Grenzobjekt. Erst $a b t=7$ wird sie aufgrund ihrer vollständigen Tilgung als solches abgelöst. Nun stellt die deutlich geringer verzinste Kreditlinie B die beste Verwendung der letzten verfügbaren Geldeinheit dar.

Im Bewertungsprogramm kann man noch einmal sehr anschaulich die Bedeutung der Barwerte am Ende des Planungshorizonts nachvollziehen, erkennbar am Kontostand (letzte Zeile): In den ersten zehn Perioden werden die Schulden von etwa 158.000 EUR auf etwa 114.000 EUR zurückgeführt. Die Tilgung der Restschuld erfolgt nur in der Darstellung auf einmal am Planungshorizont. Denn die Quelle dieser vermeintlichen Sondertilgung ist der Barwert der zukünftigen Zahlungsüberschüsse jenseits des Planungshorizonts. Er dient der Vereinfachung, um nicht alle weiteren 40 Perioden im Modell abbilden zu müssen. Sein Zinssatz repräsentiert den Grenzzinsfuß und damit auch 
Kreditgeschäfte jenseits des Planungshorizonts, weil Kredit- und Geldanlageobjekte explizit nur bis zur zehnten Periode modelliert wurden.

Die vermeintliche Mittelherkunft stammt aus dem Barwert der Zahlungsströme des Bewertungsobjekts $\left(\mathrm{g}_{\mathrm{K} 10}:+100.284 \mathrm{EUR}\right)$ und des frei verfügbaren Einkommens $\left(\mathrm{b}_{10}\right.$ : +51.842 EUR) abzgl. der Konsumentnahme (EN: -71.556 EUR). Eine Ausnahme bildet lediglich $\mathrm{I}_{9}$ (in $\mathrm{t}=10:+34.300$ EUR). Dies ist kein Barwert, sondern die Einzahlung aus dem Mathematikunterricht, der am Planungshorizont letztmalig durchgeführt wird. Die große Sondertilgung ist also, ebenso wie die plötzliche Mittelherkunft, der modellmäßig vereinfachten Darstellung einer ebenso gleichmäßigen Tilgung im Laufe der nächsten 40 Jahre geschuldet.

Die Ergebnisdarstellung mittels VOFI verdeutlicht plastisch den Vorteil von Totalmodellen. Sie erfassen das gesamte Entscheidungsfeld und lassen viele Zusammenhänge besser erkennen: Selbst wenn ein Partialmodell die theoretisch richtigen Kalkulationszinsfüße nutzen würde, wäre sein Ergebnis nur in der obersten linken Zelle des VOFI zu finden. Es würde lediglich einen Ertragswert auswerfen. Weitere Daten würden in seiner komprimierten Darstellung verlorengehen. Das Totalmodell hingegen bietet einen Gesamtüberblick aller Geldbewegungen, bestehend aus Mittelherkunft und -verwendung aller im Entscheidungsfeld vorkommenden (bzw. modellmäßig erfaßten) Objekte.

Open Access Dieses Kapitel wird unter der Creative Commons Namensnennung 4.0 International Lizenz (http://creativecommons.org/licenses/by/4.0/deed.de) veröffentlicht, welche die Nutzung, Vervielfältigung, Bearbeitung, Verbreitung und Wiedergabe in jeglichem Medium und Format erlaubt, sofern Sie den/die ursprünglichen Autor(en) und die Quelle ordnungsgemäß nennen, einen Link zur Creative Commons Lizenz beifügen und angeben, ob Änderungen vorgenommen wurden.

Die in diesem Kapitel enthaltenen Bilder und sonstiges Drittmaterial unterliegen ebenfalls der genannten Creative Commons Lizenz, sofern sich aus der Abbildungslegende nichts anderes ergibt. Sofern das betreffende Material nicht unter der genannten Creative Commons Lizenz steht und die betreffende Handlung nicht nach gesetzlichen Vorschriften erlaubt ist, ist für die oben aufgeführten Weiterverwendungen des Materials die Einwilligung des jeweiligen Rechteinhabers einzuholen.

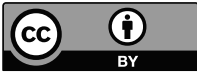

